END-TIDAL CARBON DIOXIDE IN THE DIAGNOSIS OF ACUTE PULMONARY EMBOLISM IN HOSPITALIZED ADULT PATIENTS

\begin{tabular}{c} 
A Dissertation \\
presented to \\
the Faculty of the Graduate School \\
at the University of Missouri-Columbia \\
In Partial Fulfillment \\
of the Requirements for the Degree \\
Doctor of Philosophy \\
\hline Dons. Deidre D. Wipke-Tevis, Dissertation Chair \\
DECEMBER 2018 \\
DRENTICE
\end{tabular}


The undersigned, appointed by the dean of the Graduate School, have examined the dissertation entitled

\section{END-TIDAL CARBON DIOXIDE LEVEL IN THE DIAGNOSIS OF ACUTE PULMONARY EMBOLISM IN HOSPITALIZED ADULT PATIENTS}

Presented by Donna M. Prentice, a candidate for the degree of doctor of philosophy and hereby certify that, in their opinion, it is worthy of acceptance.

Associate Professor Deidre D. Wipke-Tevis

Professor Gregory Alexander

Associate Professor Lori Popejoy

Professor Mark Milanick 


\section{DEDICATION}

My loving husband, Michael who provided much needed support, understanding and endurance for the long haul.

My children, Alex and Nicole, who were my best cheerleaders.

My parents who always encouraged me.

My Lord and Savior, Jesus Christ, whose strength provided the ability to accomplish more than I could have on my own strength. 


\section{ACKNOWLEDGEMENTS}

I would like to acknowledge the guidance and support that I have received from my dissertation chair, Dr. Wipke-Tevis. Dr. Wipke-Tevis has been a phenomenal mentor from the beginning of my doctoral journey. She had methodically guided my process in ways that I cannot adequately describe. While I was in those inevitable low spots of the journey, Dr. Wipke-Tevis provided the necessary encouragement and/or gentle nudge that would re-kindle the forward progress. She provided invaluable insight in regards to elective courses, conference attendance, research practicums and grant applications. As a result of her wisdom, I feel that I had the best possible education.

I want to also acknowledge my dissertation committee members, Drs. Alexander,

Milanick and Popejoy. They are encouraging and insightful, and have helped to form my dissertation research into the best possible study. They have been available for questions outside of scheduled committee meetings. It has been a blessing to have their guidance during my journey.

Two additional University of Missouri faculties I wish to acknowledge are Rebecca Graves and Dr. Chelsea Deroche. The librarian Rebecca Graves was always available to help with references plus she is an amazing Zotero wizard. Dr. Deroche was instrumental in ensuring the statistical analysis was precise. I am grateful to both for their help and guidance.

The radiology team at Barnes-Jewish Hospital was an integral part of helping this research occur. Joseph Lombardo and Aaron Klepner both provided data related to financial charge for the computed tomography (CT) scans, annual number of scans and 
the radiation exposure for each participant. Judith Weber and Michael Steinkruger were amazing with providing participant information when a pulmonary embolism protocol chest CT was ordered. They were enthusiastic and ever watchful for potential participants.

Finally this study was supported by funding from the Society of Critical Care Medicine Shark Bait and Toni and Jim Sullivan Endowed Research Fund for PhD Students. 
TABLE OF CONTENTS

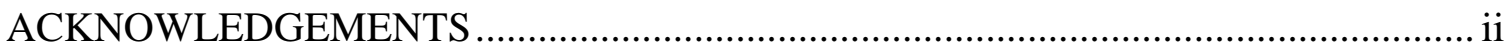

LIST OF ILLUSTRATIONS ............................................................................. vii

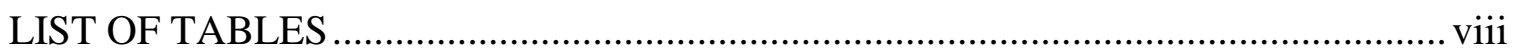

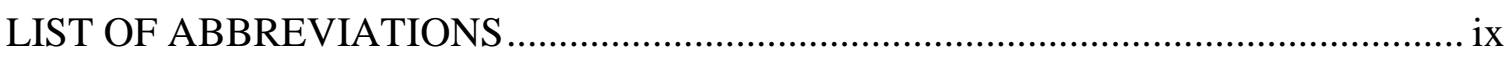

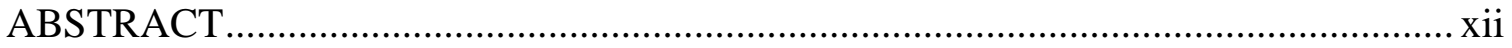

CHAPTER ONE:

Diagnosis of Pulmonary Embolism ..................................................................... 3

Overuse of CTPA Testing for PE Diagnosis ………………...................................... 3

Use of Capnography $\left(\mathrm{EtCO}_{2}\right)$ in PE Diagnostic Process .............................................. 5

Purpose

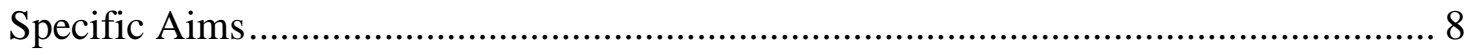

Conceptual Model ............................................................................................ 9

Significance of the Study to Nursing and Health Care Innovation.............................. 10

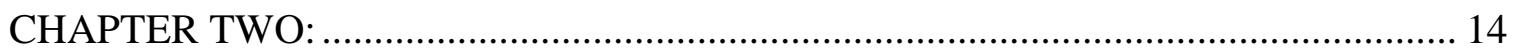

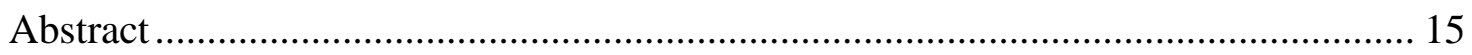

Diagnosis of Pulmonary Embolism: Following the Evidence from Suspicion to

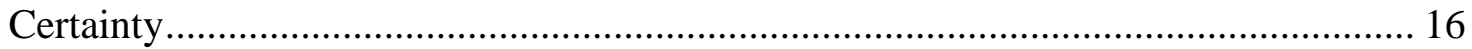

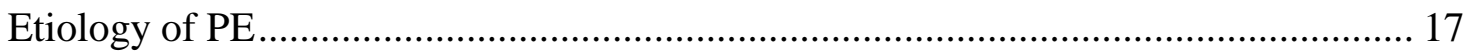

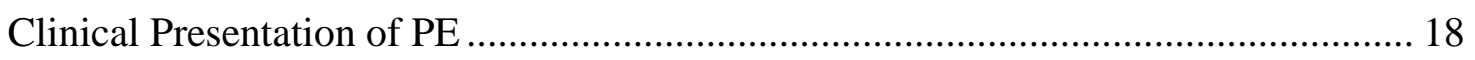

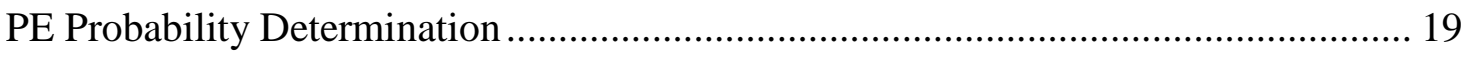

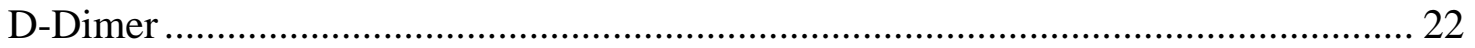

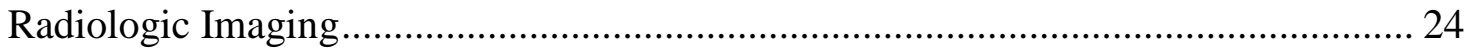

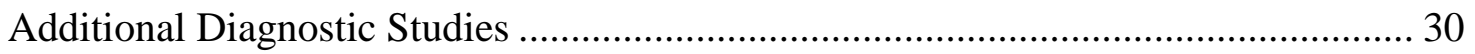

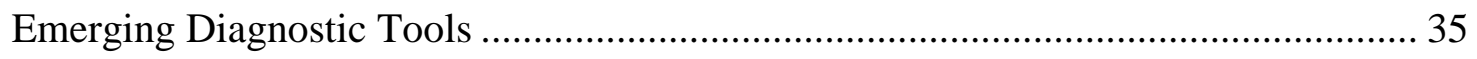

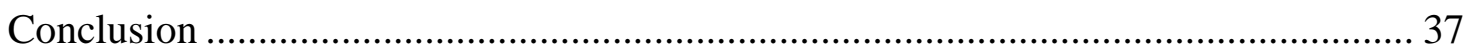

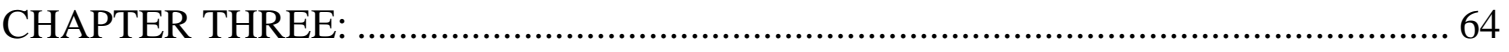

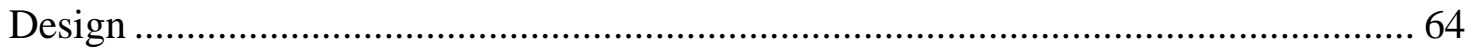

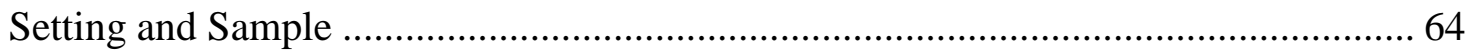

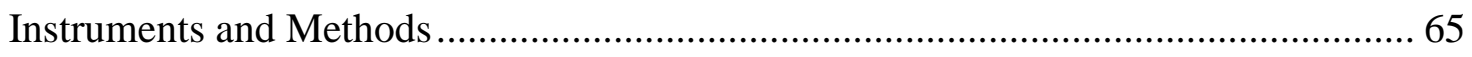

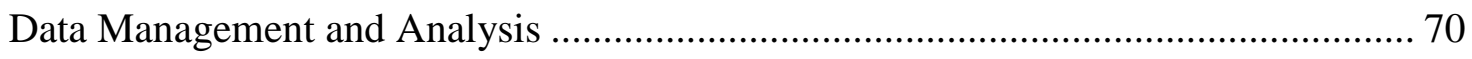

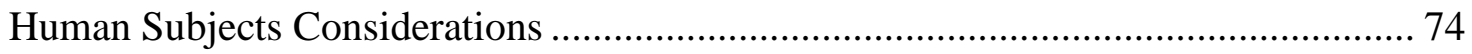




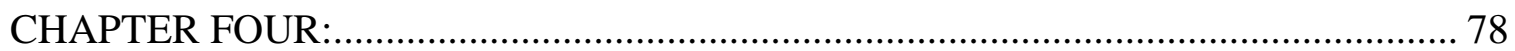

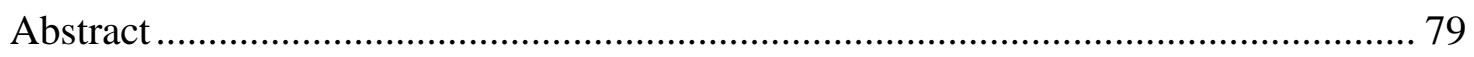

End-Tidal Carbon Dioxide Level in the Diagnosis of Acute Pulmonary Embolism in

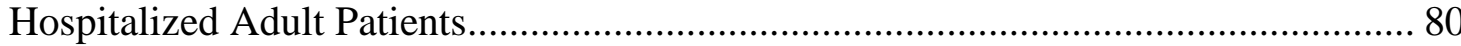

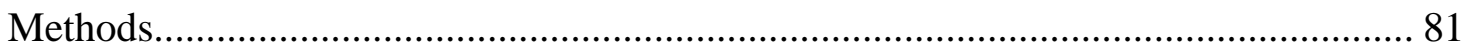

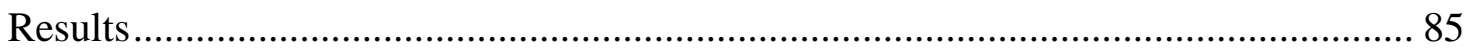

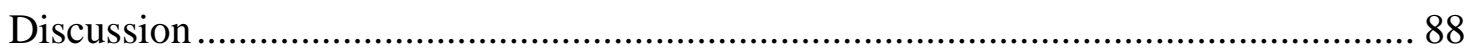

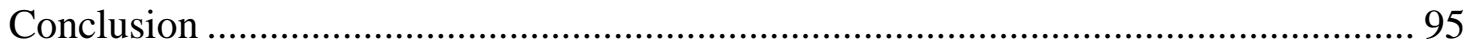

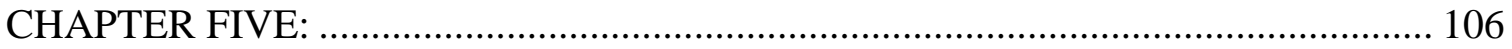

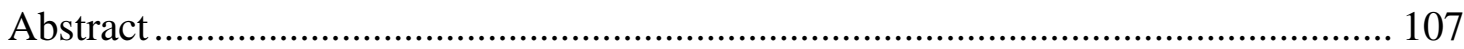

Adherence with American Collage of Physician's Best Practice Advice for Diagnosis

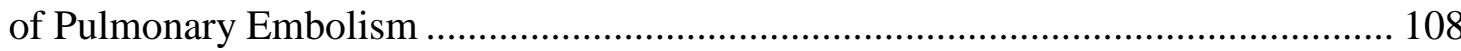

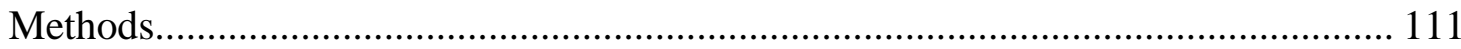

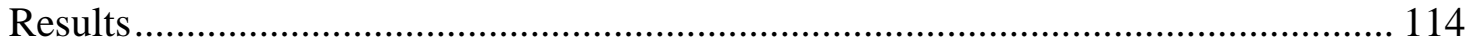

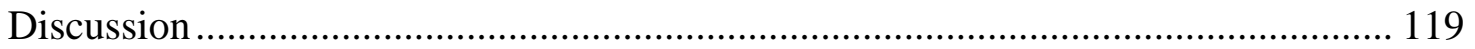

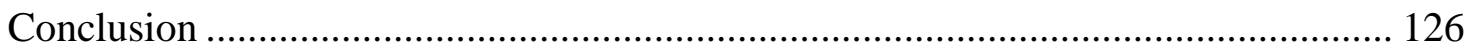

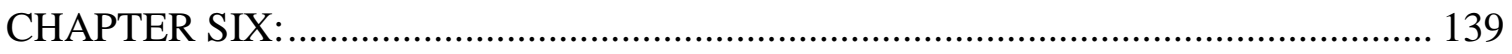

Overview of Dissertation Preparation.............................................................. 139

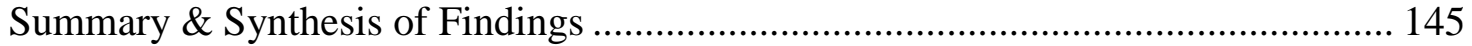

Significance of the Dissertation Work ................................................................ 149

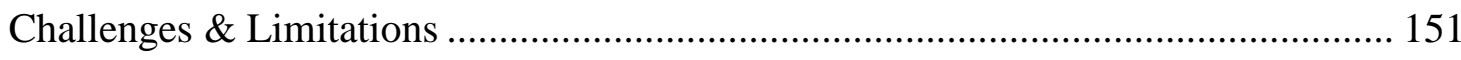

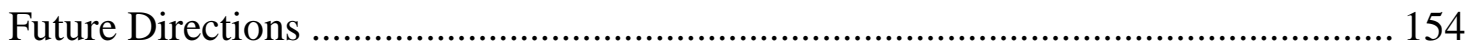

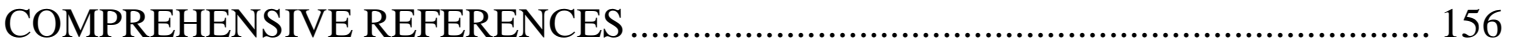

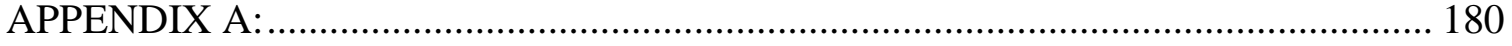

University of Missouri Human Subject Approval ................................................. 180

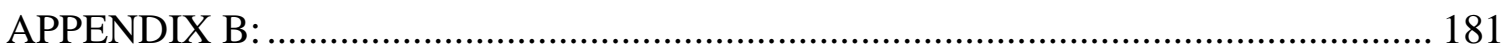

Barnes-Jewish Hospital Approval Letter ........................................................ 181

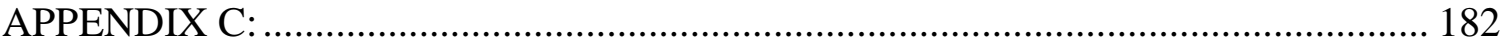

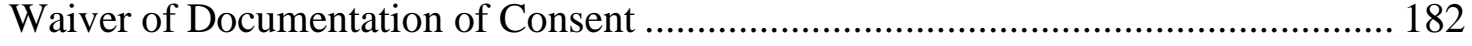

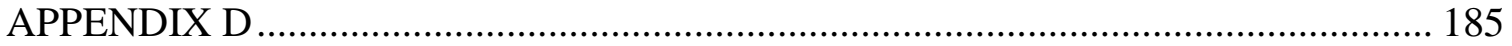

Diagnosis of PE: Data Collection Tool ......................................................... 185

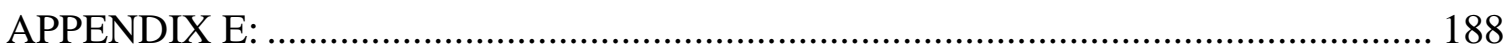


Journal of Vascular Nursing Article Acceptance................................................... 188

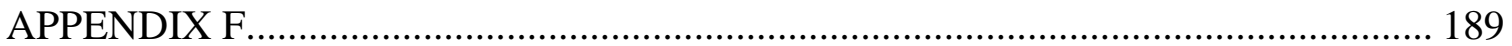

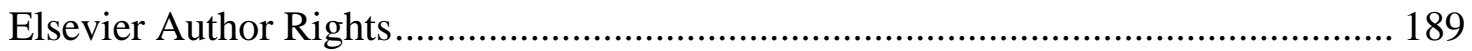

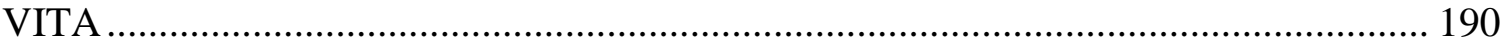




\section{LIST OF ILLUSTRATIONS}

Figure

1.1 Conceptual Model....................................................13

2.1 American College of Physicians Best Practice Advice ............................56

2.2 Pulmonary Embolism Alters Alveolar Dead Space and End Tidal Carbon Dioxide .57

4.1 ROC Curve for End Tidal Carbon Dioxide ................................ 104

5.1 Frequency of Positive Pulmonary Embolism Rule Out (PERC) Criteria by Group..137 


\section{LIST OF TABLES}

Table

Page

1.1 Summary of End-Tidal Capnography Studies in the PE Diagnostic Process ........12

2.1 Clinical Decision Rules (CDR) for the Diagnosis of Pulmonary Embolism (PE) ....58

2.2 Summary of Testing Options for Pulmonary Embolism Diagnosis ................59

4.1 Participant Demographics, Comorbidity and PE Risk Factors ....................100

4.2 PE Clinical Signs, PE Probability and Rational for CTPA .....................102

4.3 Sensitivity/ Specificity PPV/NPV for Variable $\mathrm{EtCO}_{2}$ Cut Off ..................103

5.1 PI Calculated Traditional Wells Score from Electronic Medical Record ............135

5.2 Clinical Indications for D-dime Results to be Positive .........................136 


\section{LIST OF ABBREVIATIONS}

\begin{tabular}{|c|c|}
\hline Abbrevia & \\
\hline ABG & Arterial Blood Gas \\
\hline $\mathrm{ACP}$ & American College of Physicians \\
\hline AVDS & Alveolar Dead Space \\
\hline BPA & Best Practice Advise \\
\hline BPM & Beats Per Minute \\
\hline CAD & Coronary Artery Disease \\
\hline CDR & Clinical Decision Rule \\
\hline $\mathrm{CI}$ & Confidence Interval \\
\hline CIN & Contrast-induced Nephropathy \\
\hline CMS & $\begin{array}{l}\text { Centers for Medicare and Medicaid } \\
\text { Services }\end{array}$ \\
\hline CNS & Clinical Nurse Specialist \\
\hline COPD & Chronic Obstructed Pulmonary Disease \\
\hline СТЕРН & $\begin{array}{l}\text { Chronic Thromboembolism Pulmonary } \\
\text { Hypertension }\end{array}$ \\
\hline СТА & $\begin{array}{l}\text { Computed Tomography Pulmonary } \\
\text { Angiography }\end{array}$ \\
\hline CUS & Compression Ultrasonography \\
\hline CXR & Chest X-Ray \\
\hline DBP & Diastolic Blood Pressure \\
\hline $\mathrm{dl}$ & Deciliter \\
\hline DRGs & Diagnosis Related Groups \\
\hline DVT & Deep Vein Thrombosis \\
\hline ECG & Electrocardiogram \\
\hline ED & Emergency Department \\
\hline EMR & Electronic Medical Record \\
\hline
\end{tabular}




\begin{tabular}{ll}
\hline EMS & Emergency Medical Services \\
$\mathrm{EtCO}_{2}$ & End-Tidal Carbon Dioxide \\
$\mathrm{HR}$ & Heart Rate \\
$\mathrm{HRT}$ & Hormone Replacement Therapy \\
$\mathrm{IRB}$ & Internal Review Board \\
$\mathrm{IV}$ & Intravenous \\
$\mathrm{JIT}$ & Just In Time \\
$\mathrm{LAR}$ & Legally Authorized Representative \\
$\mathrm{LE}$ & Lower Extremity \\
$\mathrm{LOS}$ & Length of Stay \\
$\mathrm{MICU}$ & Medical Intensive Care Unit \\
$\mathrm{mmHg}$ & Millimeters of Mercury \\
$\mathrm{mSv}$ & Milliserverts \\
$\mathrm{Ng}$ & Nanograms \\
$\mathrm{NPV}$ & Negative Predictive Value \\
$\mathrm{NS}$ & Not Significant \\
$\mathrm{O}_{2}$ & Oxygen \\
$\mathrm{PACO}$ & Partial Pressure of Alveolar Carbon \\
$\mathrm{PE}$ & Pixide \\
$\mathrm{PERC}$ & Pulmonary Embolism \\
$\mathrm{PI}$ & Pulmonary Embolism Rule Out Criteria \\
$\mathrm{PVCO}$ & Principal Investigator \\
\hline & Positive Likelihood Ratio \\
& Point of Care \\
& Point of Care Ultrasound \\
& Positive Predictive Value \\
& Partial Pressure Mixed Venous Carbon \\
& Dioxide \\
& Room Air \\
\hline &
\end{tabular}




\begin{tabular}{ll}
\hline ROC & Receiver Operating Characteristic \\
RQ & Research Question \\
RR & Respiratory Rate \\
RV & Right Ventricular \\
SBP & Systolic Blood Pressure \\
SCCM & Society of Critical Care Medicine \\
SD & Standard Deviation \\
TEE & Transesophageal Echocardiogram \\
TTE & Transthoracic Echocardiogram \\
V/Q & Ventilation to Perfusion \\
VTE & Venous Thromboembolism \\
Yrs & Years \\
\hline
\end{tabular}




\title{
END-TIDAL CARBON DIOXIDE IN THE DIAGNOSIS OF ACUTE PULMONARY EMBOLISM IN HOSPITALIZED ADULT PATIENTS
}

\author{
Donna M. Prentice
}

Dr. Deidre D. Wipke-Tevis, Dissertation Chair

\begin{abstract}
Pulmonary embolism (PE) causes 100,000 deaths and contributes to over 4 billion dollars of annual healthcare costs. Acute PE is a diagnostic challenge as symptoms vary widely and are often nonspecific. Definitive diagnosis requires costly confirmatory testing with computed tomography pulmonary angiogram (CTPA). CTPA risks include allergic reactions, contrast-induced nephropathy and radiation exposure. The purpose of this study is two-fold: 1) to examine the accuracy, financial impact, and harm avoidance of adding $\mathrm{EtCO}_{2}$ to the $\mathrm{PE}$ diagnostic process and 2) to evaluate clinician adherence to national guidelines (BPA) for PE diagnosis. A single center prospective, descriptive, correlational design comparing $\mathrm{EtCO}_{2}$ values to CTPA results. Medical records were reviewed to determine BPA adherence. A total of 111 patients had definitive CT results. Mean $( \pm \mathrm{SD}) \mathrm{EtCO}_{2}$ was higher for PE+ $(28 \pm 7.8)$ versus PE- $(33 \pm 8.1)$ patients $(p=.01)$. For PE exclusion, an $\mathrm{EtCO}_{2}$ cutoff value of $\geq 42 \mathrm{mmHg}$ yielded a sensitivity of $100 \%$, specificity of $12.2 \%$ and a negative predictive value of $100 \%$ and could safely eliminate 11 patients (9.9\%) from receiving CTPA. For every 6 patients assessed with $\mathrm{EtCO}_{2}$, one can be saved from CTPA. Overall BPA adherence was 0\%. Partial adherence was observed with clinician recorded clinical decisions rules in 3.6\% (4/111) and D-dimer was obtained in $10.2 \%$ (9/88). EtCO $\mathrm{Et}_{2}$ cutoff value of $\geq 42 \mathrm{mmHg}$ could decrease CTPA
\end{abstract}


scans use in $\sim 10 \%$ of adult inpatients suspected of PE eliminating exposure to CTPA risks. Lack of clinical decision support may contribute to low BPA compliance. 


\section{CHAPTER ONE:}

\section{INTRODUCTION}

Efforts to contain healthcare costs have been ongoing in the United States since the institution of the Diagnosis Related Groups (DRGs) in the early 1980s (Cacace \& Schmid, 2009). More recent cost containment efforts instituted by the Centers for Medicare and Medicaid Services (CMS) have included refusal to reimburse costs associated with selected hospital-acquired conditions (Averill, Hughes, Goldfield, \& McCullough, 2009). In their seminal article Eliminating Waste in US Healthcare, Berwick and Hackbarth (2012) proposed the novel idea of reducing waste in healthcare spending rather than reducing value added healthcare. Moreover, they identified six categories of healthcare waste (failures of care delivery, failures of care coordination, overtreatment, administrative complexity, pricing failures, and fraud and abuse) that were estimated to account for $34 \%$ of 2011 US healthcare expenditures (Berwick \& Hackbarth, 2012). As a result, eliminating unnecessary medical care, such as nonbeneficial medical tests and treatments, is not only receiving increased attention from health systems (Levinson et al., 2015) but is also considered to be ethically mandated (Brody, 2012).

Failures of care delivery are described as "waste that comes with poor execution or lack of widespread adoption of known best care processes" (Berwick \& Hackbarth, 2012, p. 1513). Barriers to adoption of clinical guidelines can arise from clinicians, patients or the healthcare system. For example, clinician-related barriers to guideline adoption include clinician resistance, lack of time, and lack of knowledge and understanding of the guideline (Gransjøen, Wiig, Lysdahl, \& Hofmann, 2018; Ryan, 
2017; Sherk \& Stojanovska, 2017). Patient-related barriers include pressure from patients to receive a particular medication, test, and/or treatments (Gransjøen, Wiig, Lysdahl, \& Hofmann, 2018). Reported healthcare system related obstacles to guideline usage include lack of guideline incorporation into the EMR, feasibility of an intervention and time constraints with lack of clinical support to apply a guideline (Ryan, 2017; Sherk \& Stojanovska, 2017).

Overtreatment is defined as "the waste that comes from subjecting patients to care that, according to sound science and the patients' own preferences, cannot possibly help them and is rooted in outmoded habits, supply-driven behaviors, and ignoring science" (Berwick \& Hackbarth, 2012. p. 1514). For example, overuse of medical testing sets up a situation where the benefit of the test is outweighed by the potential harm (Bethge et al., 2017; Morgan, Dhruva, Coon, Wright, \& Korenstein, 2018; Winchester et al., 2018). In addition to the potential harm from the actual test, overuse of testing can lead to over diagnosis and over treatment causing further harm (emotional, financial, and/or physical) and healthcare costs. There are many reasons for overuse such as ambiguous indications, lack of clinician understanding of risks or current guidelines, resistance to what is perceived as limitations to medical judgement with diagnostic algorithms, fear of litigation, overreliance on technology, accessibility to technology, cultural norms and/or a payor system that increases clinician reimbursement for testing (Bethge et al., 2017; Morgan et al., 2018; Wiener et al., 2014; Zikmund-Fisher et al., 2017). Patient's expectations that more testing and treatment is a sign of better care can pressure clinicians to order tests and therapy that they would not otherwise provide (WalshChilders \& Braddock, 2018). Additionally, health care marketing to clinicians and the 
public often touts the benefits of testing or therapy without information regarding appropriate indications and risks (Walsh-Childers \& Braddock, 2018).

\section{Diagnosis of Pulmonary Embolism}

Patients with pulmonary embolism (PE) may present with a variety of nonspecific signs and symptoms which overlap with multiple other conditions. PE signs and symptoms range from very mild to devastating severe shock states (Kline \& Kabrhel, 2015). Additionally, on occasion, the patient may be asymptomatic or present with sudden death (Kline \& Kabrhel, 2015; Konstantinides et al., 2014; Long \& Koyfman, 2016). Unfortunately, no individual sign or symptom viewed in isolation has the ability to diagnose or exclude PE (Kline \& Kabrhel, 2015; Konstantinides et al., 2014; Long \& Koyfman, 2016). Consequently, the American College of Physicians (Raja et al., 2015) has provided best practices for a standardized approach for PE diagnosis. Specifically, the stepwise approach for the hemodynamically stable (blood pressure $\geq 90 \mathrm{mmHg}$ ) patient includes: probability determination using a validated PE clinical decision rule (CDR); Pulmonary Embolism Rule Out Criteria (PERC) assessment; d-dimer testing; and imaging with computed tomography pulmonary angiography (CTPA) (Raja et al., 2015). A detailed, state of the art, clinical review of the PE diagnostic process, including each step of the 2015 American College of Physicians Evaluation of Patients with Suspected Acute Pulmonary Embolism Best Practice Advice, is provided in Chapter 2.

\section{Overuse of CTPA Testing for PE Diagnosis}

Computed tomography pulmonary angiogram (CTPA) is "increasingly used as the first and only test for suspected PE” (Chandra, Sarkar, Chandra, Ginsberg, \& Cohen, 
2013, p. 1). Current estimates are that only $10 \%$ of patients undergoing a CTPA will be positive for PE, suggesting an opportunity exists to decrease not only unnecessary radiologic testing but also healthcare costs (Parikh et al., 2015; Perelas et al., 2015b; Perera, Aggarwal, Scott, \& Cocks, 2017; van Es et al., 2013). The usefulness of the diagnostic test must be weighed against the risk. While offering the benefit of a definitive diagnosis, the CTPA carries significant short- and long-term health risks to the patient (Levin et al., 2015).

Short-term health risks associated with CTPA include allergic reaction to the contrast substance and contrast-induced nephropathy (CIN) (Kooiman et al., 2012; Le Roux et al., 2014; McDonald et al., 2014; Traub et al., 2013). While extremely rare, life threatening reactions to intravenous contrast do occur in $0.1 \%$ of the population with an unexpected death rate from the allergic reaction being 1 in 75,000 (McDonald et al., 2014). Additionally, contrast-induced nephropathy (CIN) impacts $2-7 \%$ of the population that undergo CTPA (Alhassan, Sayf, Arsene, \& Krayem, 2016; Kooiman et al., 2012; McDonald et al., 2014; Traub et al., 2013). While most patients will only experience a brief increase in creatinine, others may experience acute renal injury (Alhassan et al., 2016; Kooiman et al., 2012; McDonald et al., 2014) which, in the worst case scenario, may result in costly dialysis treatments. Patients with chronic kidney disease, diabetes mellitus, nephrotoxic medication, hypertension, dehydration and heart failure are at increased risk for CIN (Kooiman et al., 2012). Moreover, given the continuing aging of our society, CIN prevalence associated with contrast may increase over time since older adults have an increase in the risk factors associated with CIN. 
Long-term health risks associated with CTPA are due to radiation exposure (Brenner, 2012; Dainiak, 2013; Le Roux et al., 2014; Parikh et al., 2015; Rohner et al., 2013; Schembri, Miller, \& Smart, 2010). CTPA exposes the patient to a significant amount of ionizing radiation (range 10-70 millisieverts (mSv)) (Dainiak, 2013; Dogan et al, 2015). Damage to DNA from radiation exposure may result in biologic effects many years following the exposure (Schembri et al., 2010). Every exposure to ionizing radiation leads to cumulative DNA damage further increasing the risk of cancer (Schembri et al., 2010). The younger the age at exposure, frequency of repeated tests, along with genetic factors, may further increase the risk of radiation injury. Additionally, sensitivity to radiation varies by organs. For example, breasts are particularly radiosensitive. Of note, in younger women, the CTPA exposes the breast to 10 to $70 \mathrm{mSv}$ which is equal to 10 to 25 mammograms or 100 to 400 chest radiographs (Dogan et al., 2015; Schembri et al., 2010). Importantly, one recent study found a statistically significant increase $(\mathrm{p}<0.004)$ in the number of CTPAs completed on younger women less than age 40 between 2000 and 2010 (Chandra et al., 2013). Thus, avoiding unnecessary CTPA may be especially important in younger women to minimize the risk of cancer in the future from cumulative medical radiation exposure.

\section{Use of Capnography $\left(\mathrm{EtCO}_{2}\right)$ in PE Diagnostic Process}

Adding a low cost, non-invasive step to the PE diagnostic process that lowers the number of people who need to undergo CTPA would be a significant contribution. Given that PE causes an increase in alveolar dead space (AVDS), a screening test that evaluates the presence or absence of increased AVDS could meet this need. Despite the physiology of AVDS being well established, it has only been in recent history that bedside clinical 
assessment of AVDS has become practical. Specifically, the development of bedside $\mathrm{EtCO}_{2}$ has made assessment of AVDS possible. $\mathrm{EtCO}_{2}$ equipment is simple to use and easily available to the clinician. In a normal lung, $\mathrm{EtCO}_{2}$ will approximate arterial carbon dioxide $\left(\mathrm{PaCO}_{2}\right)$ at the end of exhalation. When AVDS ventilation increases, the amount of exhaled carbon dioxide decreases, disproportionally lowering $\mathrm{EtCO}_{2}$ compared to $\mathrm{PaCO}_{2}$ (West \& Luks, 2016). Thus, based on PE pathophysiology, by definition, a normal AVDS excludes PE presence. Moreover, $\mathrm{EtCO}_{2}$ assessment can detect differences between patients with and without PE by identifying nonspecific AVDS increases.

One published meta-analysis (Manara, D'hoore, \& Thys, 2013) examined the usefulness of capnography in the $\mathrm{PE}$ diagnostic process using $\mathrm{EtCO}_{2}$ studies published between 1990 through 2010 . The 14 pooled studies indicated the sensitivity of an $\mathrm{EtCO}_{2}$ assessment for PE was $80 \%$ (95\% confidence interval (CI) 76 to $83 \%$ ) and a specificity of $49 \%$ (95\% CI 23 to 45\%) with a negative likelihood ratio 32\% (95\% CI 23 to 45\%). The cut-off values for $\mathrm{EtCO}_{2}$ were not calculated. Nonetheless, the meta-analysis suggests that $\mathrm{EtCO}_{2}$ may be a good additional assessment to use in the PE diagnostic process to help exclude PE (Manara et al., 2013).

To date, five studies have purposely evaluated the usefulness of absolute $\mathrm{EtCO}_{2}$ assessment, the simplest capnography method, in the PE diagnostic process (Table 1.1; Fabius, Eijsvogel, van der Lee et al, 2016; Hemnes et al., 2010; Ramme et al., 2016; Riaz \& Jacob, 2014; Rumpf, Krizmaric, Grmec et al., 2009). Four studies found a statistically significant difference between the absolute $\mathrm{EtCO}_{2}$ of patients with and without $\mathrm{PE}$ (Hemnes et al., 2010; Ramme et al., 2016; Riaz \& Jacob, 2014; Rumpf et al., 2009). Specifically, patients without $\mathrm{PE}$ had a significantly higher $\mathrm{EtCO}_{2}$ than patients with $\mathrm{PE}$. 
Although Fabius and colleagues (2016) did not find a statistically significant difference in absolute $\mathrm{EtCO}_{2}$ between patients with and without PE, it was a small pilot study $(\mathrm{n}=30)$, likely lacked sufficient power to find a statistical difference, and utilized a volumetric assessment of absolute $\mathrm{EtCO}_{2}$ rather than the time assessment method used by others. While four recent studies found absolute $\mathrm{ETCO}_{2}$ to be statistically different between PE positive and negative patients, a consistent cutoff score has not been established.

\section{Purpose}

Thus, the purpose of this dissertation project was two-fold: 1) to examine the accuracy, financial, and potential risk avoidance of adding $\mathrm{EtCO}_{2}$ to the $\mathrm{PE}$ diagnostic process and 2) to evaluate clinician adherence to national guidelines for PE diagnosis. As part of this dissertation, three manuscripts are presented. One manuscript under review, Diagnosis of Pulmonary Embolism: Following the Evidence from Suspicion to Certainty (Prentice \& Wipke-Tevis, In Press 2018), was developed as part of the advanced research practicum Donna Prentice completed with Dr. Deidre D. Wipke-Tevis. The second manuscript, Exhaled Carbon Dioxide Level in the Diagnosis of Acute Pulmonary Embolism in Hospitalized Adult Patients, was developed with data from the original and significant investigation completed by Donna Prentice. The third manuscript, Adherence with American Collage of Physician's Best Practice Advice for the Diagnosis of Pulmonary Embolism also was developed with data from the original and significant investigation completed by Donna Prentice. Specific aims and research questions are below. 
Specific Aims

Specific Aim 1: To compare absolute end-tidal carbon dioxide $\left(\mathrm{EtCO}_{2}\right)$ levels in the PE diagnostic process for hemodynamic stable hospitalized adult patients.

Research Question (RQ) 1a: What is the level of $\mathrm{EtCO}_{2}$ that provides the best sensitivity and specificity for the exclusion of an acute PE in hospitalized adult patients?

RQ 1b: What is the negative predictive value of an $\mathrm{EtCO}_{2}$ level in hemodynamically stable hospitalized adult patients being evaluated for an acute PE? Specific Aim 2: To examine the financial impact of the use of $\mathrm{EtCO}_{2}$ during the PE diagnostic process for hemodynamically stable hospitalized adult patients.

RQ 2a: What is the estimated institutional cost for adding $\mathrm{EtCO}_{2}$ to the $\mathrm{PE}$ diagnostic process?

RQ 2b: What is the estimated healthcare cost savings (billable charge) for avoiding unnecessary CTPA for patients without a PE?

Specific Aim 3: To examine adherence to the current best practice regarding the PE diagnostic process for hemodynamically stable hospitalized adult patients.

RQ 3a: What is the overall rate of adherence to the 2015 American College of Physicians guidelines for the diagnosis of acute PE?

RQ 3b: What is the rate of adherence to the 2015 American College of Physicians six individual best practice advice statements for the diagnosis of acute PE? 


\section{Specific Aim 4: To quantify the actual and potential harm associated with unnecessary use of CTPA in the PE diagnostic process for hemodynamically stable hospitalized adult patients.}

RQ 4a: What is the frequency of allergic reactions for hospitalized adult patients undergoing unnecessary CTPA?

RQ 4b: What is the frequency of contrast induced nephropathy for hospitalized adult patients undergoing unnecessary CTPA?

RQ 4c: How much radiation exposure is avoided by eliminating unnecessary CTPA?

\section{Conceptual Model}

Figure 1.2 depicts the conceptual model for this dissertation study. The model was based on the current 2015 American College of Physicians Evaluation of Patients with Suspected Acute Pulmonary Embolism Best Practice Advice recommendations (Raja et al, 2015). The concepts of interest in this study are in bolded text in shaded boxes. Specifically, this dissertation study examined the potential benefit of adding $\mathrm{EtCO}_{2}$ assessment for the evaluation of patients with suspected PE with: a) low pretest probability plus PERC positive, and b) intermediate pretest probability. The proposed $\mathrm{EtCO}_{2}$ assessment, in addition to the existing recommended d-dimer testing, will strengthen the diagnostic process by providing additional physiologic data to support the exclusion of a PE prior to imaging. A negative $\mathrm{EtCO}_{2}$ assessment (i.e. higher $\mathrm{EtCO}_{2}=$ normal AVDS) along with a negative d-dimer result (i.e. no recent clotting) could safely exclude PE diagnosis and eliminate imaging. A negative $\mathrm{EtCO}_{2}$ assessment (normal AVDS) in the presence of a positive d-dimer (non-specific recent clotting) could 
also exclude a PE diagnosis and eliminate imaging since d-dimer may be elevated for other reasons not related to PE (e.g. recent surgery, procedure or injury or inflammation). A positive $\mathrm{EtCO}_{2}$ assessment (increased AVDS) along with a negative d-dimer (no recent clotting) could exclude a PE diagnosis since AVDS may be increased due to other causes such as low cardiac output or chronic obstructive lung disease. A patient with both a positive $\mathrm{EtCO}_{2}$ assessment (increased AVDS) and a positive D-dimer (non-specific recent clotting) has a high likelihood of having a PE and would be indicated for further imaging. In this study, $\mathrm{EtCO}_{2}$ is the primary variable of interest and will be measured on all subjects; whereas, D-dimer will only be available if ordered by the treating clinician.

\section{Significance of the Study to Nursing and Health Care Innovation}

The vague and nonspecific signs and symptoms of patients presenting with PE create a challenge for prompt diagnosis and institution of appropriate PE treatment. Not recognizing and treating PE can lead to death, while unnecessary PE testing and treatment can lead to complications associated with radiologic testing (allergic reaction, acute kidney injury, cumulative radiation exposure risks) and anticoagulation (bleeding) as well as unnecessary healthcare costs. Current best practices of using pre-test probability assessment (CDR and/or clinician gestalt) with D-dimer testing excludes PE in about $28 \%$ of patients undergoing the PE diagnostic process (van Es et al., 2017).Given that only $10 \%$ of patients (Parikh et al., 2015; Perelas et al., 2015b; Perera et al., 2017; van Es et al., 2013) undergoing the PE diagnostic process test positive for PE using CTPA, an opportunity exists to exclude more patients prior to performing a CTPA. Clinicians need an additional step in the early diagnostic process to further exclude low risk patients from the likelihood of having PE. The method needs to be easy to use with 
rapidly obtained results. The addition of an AVDS assessment with $\mathrm{EtCO}_{2}$ may meet this challenge. 
Table 1.1. Summary of End-Tidal Capnography Studies in the PE Diagnostic Process.

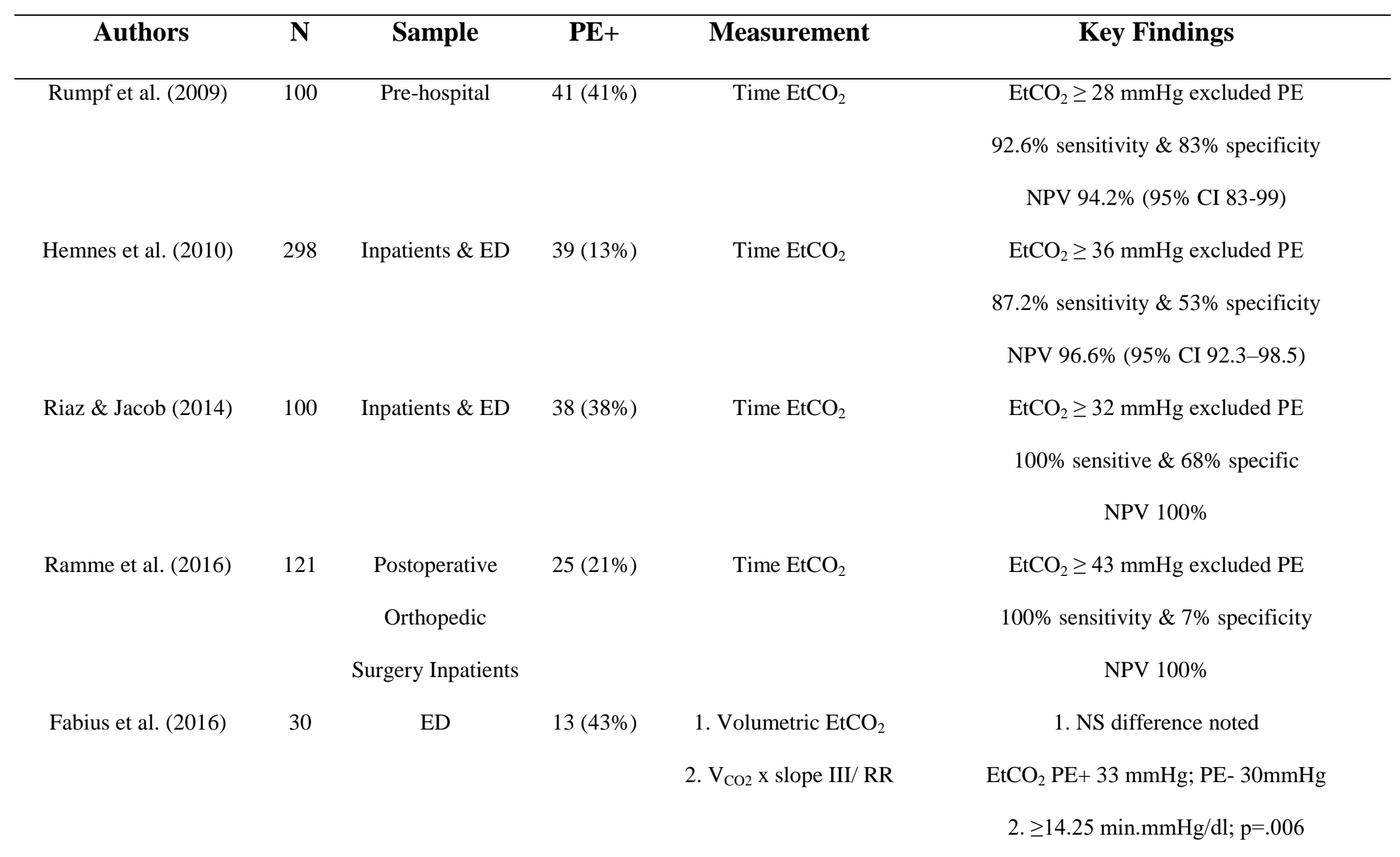

Pulmonary embolism (PE); PE Positive (PE+); PE Negative (PE-) Negative predictive value (NPV); end-tidal carbon dioxide $\left(\mathrm{EtCO}_{2}\right)$; Not Significant (NS); Emergency Department (ED); Minute (min); Millimeters of Mercury (mmHg); Deciliter (dl); Confidence Interval (CI) 
Figure 1.1. Conceptual Model

Patients with suspected

PE

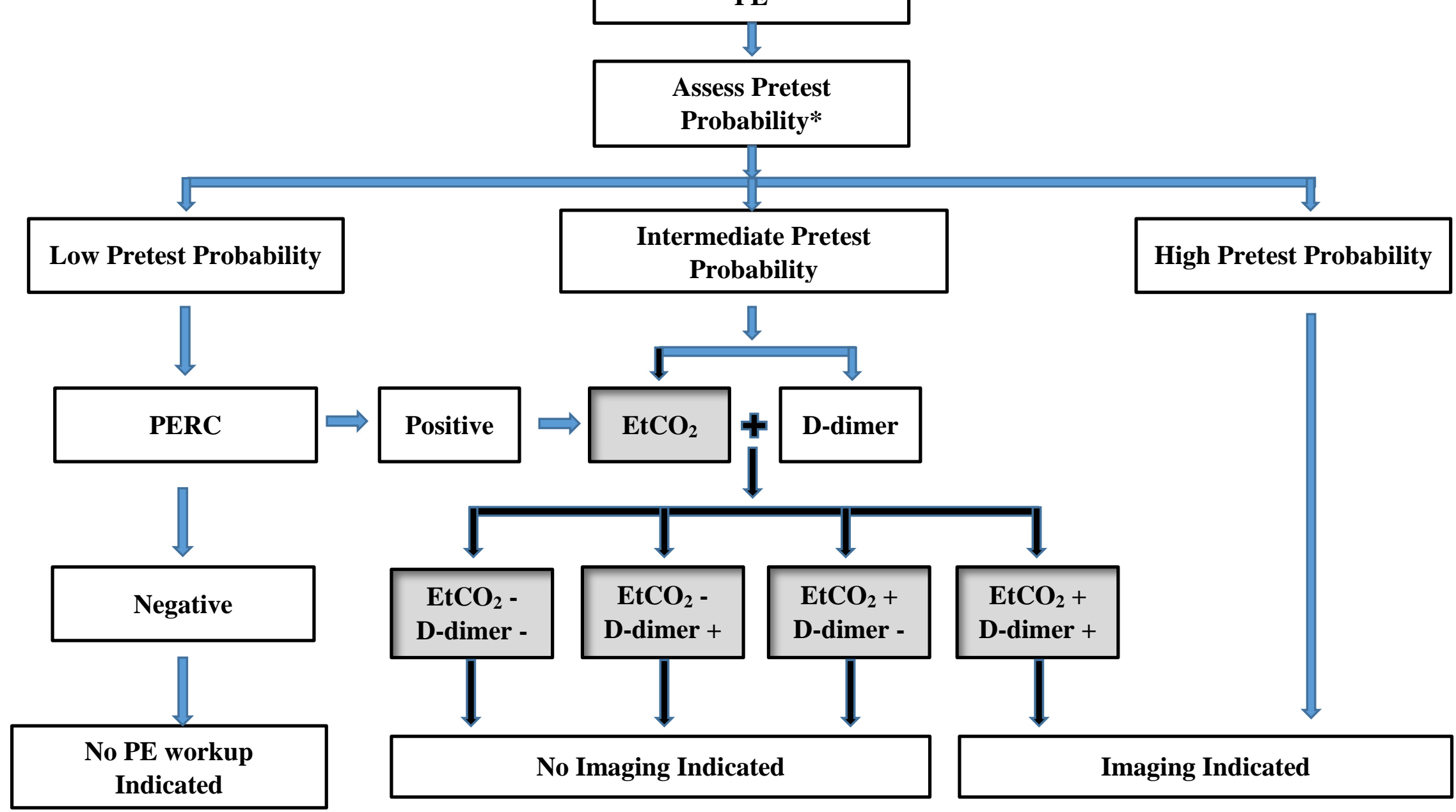

$\mathrm{PE}=$ Pulmonary Embolism; $\mathrm{EtCO}_{2}$ = End-tidal Carbon Dioxide; PERC = Pulmonary Embolism Rule-Out Criteria

*Using either a clinical decision rule or clinician gestalt; adapted with permission from the American College of Physicians clinical guideline (Raja et al, 2015) 


\section{CHAPTER TWO:}

\section{DIAGNOSIS OF PULMONARY EMBOLISM: FOLLOWING THE EVIDENCE FROM SUSPICION TO CERTAINTY}

Prentice, D., \& Wipke-Tevis, D. D. (Accepted for Publication 10/01/2018). Diagnosis of pulmonary embolism: Following the evidence from suspicion to certainty. Journal of Vascular Nursing (Appendix E and F). 


\begin{abstract}
Accurately, timely and cost effective identification of pulmonary embolism (PE) remains a diagnostic challenge. This article reviews the PE diagnostic process with a focus on the best practice advice from the American College of Physicians. Benefits and risks of each diagnostic step are discussed. Emerging diagnostic tools, not included in the algorithm, are briefly reviewed.
\end{abstract}

KEY WORDS: Pulmonary embolism (PE), probability determination, diagnostic process, best practice advice

\title{
HIGHLIGHTS:
}

- Current clinical guidelines recommend using a validated clinical decision rule

- Radiologic imaging provides the most accurate and reliable diagnosis of PE

- CTPA carries significant risks related to radiation and IV contrast exposure

Emerging diagnostic tools may minimize CTPA overuse in the future 


\section{Diagnosis of Pulmonary Embolism: Following the Evidence from Suspicion to Certainty}

Pulmonary embolism (PE) is the third most common cardiovascular disease (LaMori, Shoheiber, Mody, \& Bookhart, 2015) with an estimated annual incidence between 60 and 79 cases per 1000,000 persons (Smith, Geske, McNamara, Waterer, \& Wunderink, 2013). Since up to a third of individuals with a PE do not have any symptoms, these numbers likely underestimate the problem (Stein, Matta, Musani, \& Diaczok, 2010). For example, one recent study found the prevalence of silent PE in patients with calf vein deep vein thrombosis (DVT) to be $13.1 \%$ (Hughes, Stein, \& Matta, 2014). Regardless of biological sex, DVT and PE incidence increase dramatically with age and obesity (Puurunen et al., 2016). Moreover, the longitudinal community based Framingham study found a venous thromboembolism (VTE) hazard ratio of 1.69 per every increase in age by 10 years and a VTE hazard ratio of 1.88 for those with a body mass index greater than $30 \mathrm{~kg} / \mathrm{m}^{2}$ (Puurunen et al., 2016). Given the obesity epidemic and growing aging population, PE prevalence is projected to increase (Puurunen et al., 2016; Smith et al., 2016).

Survival following PE may lead to chronic health problems. Residual pulmonary vascular occlusion due to unresolved thrombus occurs in $20 \%$ of PE survivors (Planquette et al., 2016). Individuals with unresolved thrombus are at risk of developing chronic thromboembolic pulmonary hypertension (CTEPH), which occurs in $1-4 \%$ of people who survive the initial PE (Kahn et al., 2014; Klok et al., 2016). Current guidelines (Konstantinides et al., 2014; Raja et al., 2015) do not address follow up 
screening for CTEPH; thus, the exact percentage of CTEPH is unknown (Klok et al., 2016). Prompt diagnosis and early initiation of evidence-based treatment is essential to help decrease PE related morbidity and mortality.

Given that patients with a PE can present with a wide variety of signs and symptoms - ranging from asymptomatic to non-specific to sudden death, a prompt, accurate diagnosis can be challenging (Aschermann \& Widimský, 2015; Konstantinides et al., 2014; Lavorini et al., 2013). Delay in treatment increases mortality, so timeliness is important (Kline \& Kabrhel, 2015; Lavorini et al., 2013; Raja et al., 2015). Unfortunately, the PE diagnostic process carries risks from radiation and contrast dye exposure (Brenner, 2012; Le Roux et al., 2014; McDonald et al., 2014; Traub et al., 2013). Also, once a PE is diagnosed, treatment creates bleeding risks from anticoagulation and/or thrombolytic therapy (Fesmire et al., 2011; Kearon et al., 2016; Levin et al., 2015). Indeed, untreated PE mortality can approach $25 \%$ whereas PE mortality with therapy is $8 \%$ (Kahn et al., 2014; Smith et al., 2016). Accordingly, the goal for all patients is a quick, accurate diagnosis while minimizing risks. Thus, this article will review the current best evidence (Figure 2.1) for the PE diagnostic process and discuss emerging PE diagnostic tools.

\section{Etiology of PE}

Both PE and DVT comprise the same disease process known as VTE. A PE is the blockage of one of more branches of the pulmonary artery resulting in lack of perfusion to the alveoli (West \& Luks, 2016). The risk of developing a thrombus is often described with Virchow's triad, which includes venous stasis, endothelial injury and hyper- 
coagulability of the blood (Stone et al., 2017). Venous stasis, pooling or slowing of venous blood flow, occurs with immobility, calf muscle weakness, and ankle/leg trauma. Endothelial injury arises from blood vessel injury, trauma, instrumentation, or the shear force associated with hypertension. Hyper-coagulability is associated with cigarette smoking, hormone therapy, certain genetic conditions, cancer, obesity, and pregnancy (Dybowska et al., 2015; Konstantinides et al., 2014; Lippi, Danese, Favaloro, Montagnana, \& Franchini, 2015). Venous thrombi usually attach to the vessel at a site of injury and/or stasis and extend in the direction of blood flow.

When a thrombus dislodges, it embolizes to another location until it cannot pass any further causing vessel occlusion. Most PE arise from leg DVT (Lavorini et al., 2013; Marshall, Mathews, \& Siegel, 2011). Embolization risk is greater with proximal, above the knee, DVT versus distal, below the knee, DVT (Kline \& Kabrhel, 2015; Piovella \& Iosub, 2016). Non-thrombotic PE is rare and results from embolization of amniotic fluid, tumor or fat particles, gas or foreign material (Montagnana, Cervellin, Franchini, \& Lippi, 2011). Regardless of etiology, a PE impairs perfusion of the pulmonary vascular bed (West \& Luks, 2016). As a result, alveolar dead space (AVDS), an area of the lung ventilated without perfusion, increases (Figure 2.2).

\section{Clinical Presentation of PE}

PE diagnosis is a step wise process that begins with the healthcare provider's evaluation of the clinical presentation. Patients with PE may present with a variety of nonspecific signs and symptoms which overlap with multiple other conditions and range from very mild to devastating severe shock states (Kline \& Kabrhel, 2015; 
Konstantinides et al., 2014; Raja et al., 2015). The most common PE symptoms include pleuric and nonpleuric chest pain, sudden onset of dyspnea, leg pain, fatigue, anxiety and hemoptysis (Kline \& Kabrhel, 2015; Konstantinides et al., 2014; Raja et al., 2015). Unfortunately, these symptoms are not unique to PE. For example, in a recent observational study of emergency department patients (ED), Kelly and colleagues (2017) found that although dyspnea is observed in $5.2 \%$ of all ED patients; only $1.2 \%$ of those patients were definitively diagnosed with a PE.

Clinical signs of PE may include electrocardiographic changes, leg swelling, low end tidal $\mathrm{CO}_{2}$, new onset atrial arrhythmias, hypotension, severe right heart failure (sudden shortness of breath, pink sputum, atrial fibrillation, severe weakness/fatigue, tachycardia, low cardiac output), syncope, and/or tachypnea (Kline \& Kabrhel, 2015; Konstantinides et al., 2014; Omar et al., 2017). Likewise, these signs are not exclusive to PE. For instance, although syncope is associated with acute submassive PE (Omar et al., 2017; Oqab, Ganshorn, \& Sheldon, 2017), syncope is only observed in about $12.3 \%$ of acute PE patients (Omar et al., 2017; Oqab et al., 2017). Indeed, no PE sign or symptom viewed in isolation has the ability to diagnose or exclude PE (Kline \& Kabrhel, 2015; Konstantinides et al., 2014; Raja et al., 2015). Nonetheless, clinical presentation, while nonspecific, is the foundation for determining the need for further diagnostic testing.

\section{PE Probability Determination}

Current clinical guidelines (Konstantinides et al., 2014; Raja et al., 2015) recommend using a validated clinical decision rule (CDR) to assist with the $\mathrm{PE}$ diagnostic process. Without a PE CDR to assess probability, the clinician would use their 
gestalt, or best guess based on prior experience, to determine PE likelihood. While accuracy of an experienced clinician's gestalt may be similar to that of a CDR (Kline \& Kabrhel, 2015; Lucassen et al., 2011), a CDR provides a standardized approach for less experienced clinicians or those who infrequently evaluate patients for suspected PE (Raja et al., 2015). Specifically, a PE CDR quantifies signs and symptoms to determine PE probability and need for further testing (Raja et al., 2015; Sherk \& Stojanovska, 2017). Ultimately, the PE CDR provides a numerical score that categorizes the patient's PE likelihood. A patient with an intermediate or high probability CDR score will require further diagnostic testing (Konstantinides et al., 2014; Raja et al., 2015).

Multiple PE CDRs have been developed; some have been revised, modified and/or simplified; and some are referred to by more than one name. Clinicians should note that Wells created two CDRs-- a DVT CDR (Wells et al., 1997) and a PE CDR (Wells, 1998). For this article, we only address PE CDRs. Table 2.1 provides an in-depth comparison of the indicators, scoring, and probability interpretation of the most commonly utilized PE CDRs.

Both the Wells and Geneva PE CDRs are considered valid tools for determining pretest probability (Raja et al., 2015); however, neither is considered "better" at PE risk stratification (Douma et al., 2011; Lucassen et al., 2011). Indeed, Douma and colleagues (2011) determined that when the PE CDR results were combined with a normal highly sensitive d-dimer test, accuracy indexes (sensitivity [all were 99.5\%], specificity [range 29-31\%] and negative predictive values [range 99.4-99.5\%]) were quite similar. 
Furthermore, simplified versions of the Wells and Geneva improved usage as clinicians could easily calculate the simplified CDRs from memory (Douma et al., 2011).

The Pulmonary Embolism Rule-Out Criteria (PERC), the newest CDR, is unique in that it was purposefully created to assist with identifying patients at very low PE risk and excluding them from a PE diagnosis (Kline, Mitchell, Kabrhel, Richman, \& Courtney, 2004). A patient is determined to have a low clinical probability of PE if all nine of the PERC criteria (Table 2.1) are absent; thus, a PE diagnosis is excluded and further diagnostic testing is avoided (Kline \& Kabrhel, 2015; Kline, Peterson, \& Steuerwald, 2010). A meta-analysis of 12 studies involving 14,844 patients from seven countries confirmed the safety of the PERC for use in a clinically low risk population, showing the risk of missed PE was only $0.3 \%$ with a pooled sensitivity of $97 \%$ and a pooled specificity of 22\% (Singh, Mommer, Erwin, Mascarenhas, \& Parsaik, 2013). Of note, the PERC has not been validated in hospitalized patients (Sherk \& Stojanovska, 2017) and should only be used for patients considered very low risk as determined by clinician gestalt or a validated CDR (Konstantinides et al., 2014; Raja et al., 2015).

To provide an accurate and reliable assessment, a CDR must be completed correctly. For example, one study evaluated the interrater reliability of the PERC and Wells PE CDR and found clinician disagreement occurred with the following PE indicators: unexplained hypoxia, immobilization, and alternative diagnosis less likely than PE (Nordenholz et al., 2007). While the CDR provides a valuable first step in the diagnosis of PE, CDR use adherence has not been methodically evaluated (Sherk \& Stojanovska, 2017). Factors leading to CDR nonadherence may be lack of education 
about CDRs, resistance to "cookbook" approaches to differential diagnosis, and lack of electronic medical record (EMR) support (Sherk \& Stojanovska, 2017). To facilitate CDR adherence, healthcare agencies need to select a single CDR to be utilized, provide education to the medical and nursing staff on correct usage and interpretation of the CDR, and incorporate the CDR into the EMR (Raja et al., 2015; Sherk \& Stojanovska, 2017).

While the CDRs provide a valuable first step in the PE diagnostic process, they also have limitations. Validity testing is limited within certain populations. Specifically, CDRs need more validation in elderly, hospitalized and pregnant patient populations (Konstantinides et al., 2014; Tromeur, van der Pol, Klok, Couturaud, \& Huisman, 2017).

\section{D-Dimer}

Current clinical guidelines (Konstantinides et al., 2014; Raja et al., 2015) recommend using D-dimer testing in patients who do not meet all of the PERC exclusion criteria and those with an intermediate PE probability score from a Wells or Geneva CDR. D-dimer is a fibrin degradation product, a fragment released into the circulation during fibrinolysis. The quantitative method for measuring d-dimer is more accurate than a qualitative laboratory analysis (Riley, Gilbert, Dalton, Pai, \& McPherson, 2016). The quantitative analysis D-dimer will be positive if the value is $\geq 500$ nanograms per milliliter (ng/ml) and negative if the value is $\leq 499 \mathrm{ng} / \mathrm{ml}$ (Riley et al., 2016).

D-dimer is elevated (i.e. positive D-dimer) in many normal and pathologic conditions where a thrombus is present such as acute myocardial infarction, acute thromboembolic stroke, disseminated intravascular coagulation, DVT, PE, post-surgery, 
and post trauma (Kline et al., 2012; Riley et al., 2016). Additional non-thrombotic conditions that can lead to a positive d-dimer include acute kidney injury, advanced age (> 50 years old), cancer, heart failure, hemodialysis, high rheumatoid factor, inflammation, pregnancy and post-partum, sepsis, and severe liver disease (Chopra, Doddamreddy, Grewal, \& Kumar, 2012). Due to the large number of false positive results, d-dimer utility is limited to patients who do not have a clinical condition that will stimulate the fibrinolytic system (Chopra et al., 2012; Konstantinides et al., 2014; Raja et al., 2015; Riley et al., 2016). While a positive d-dimer can be useful in the PE diagnostic process, it is not sufficient in and of itself to diagnosis a PE. Consequently, patients with an intermediate CDR score and a positive d-dimer will require further testing to definitively determine PE presence or absence (Konstantinides et al., 2014; Raja et al., 2015).

In contrast, a negative d-dimer is very sensitive for absence of a blood clot (Riley et al., 2016). A false negative d-dimer occurs in $<1 \%$ of patients but may be seen if the blood sample is drawn too soon after a clot has formed ( $<12$ hours), too long following clot formation (> 1 week), after the initiation of anticoagulation or in patients with chronic PE (Kline \& Kabrhel, 2015; Lippi et al., 2015; Youssf, Ismail, ElGhamry, \& Reyad, 2014). A Cochrane review, several investigative teams, and the current clinical guidelines have all concluded that a low PE probability score on a validated CDR when used in combination with a negative, high sensitivity d-dimer test can safely exclude PE with a negative predictive value (NPV) of 95\% (Crawford et al., 2016; Douma et al., 2011; Kline et al., 2012; Lapner \& Kearon, 2013; Lucassen et al., 2011; Raja et al., 2015; Riley et al., 2016). That said, recent research suggests that clinicians often order 
expensive and unnecessary radiologic tests such as computed tomography pulmonary angiography (CTPA) even in presence of a negative d-dimer (Chandra, Sarkar, Chandra, Ginsberg, \& Cohen, 2013; Parikh et al., 2015; Perelas et al., 2015; Yin et al., 2012).

Limitations of d-dimer testing need to be acknowledged. D-dimer requires a venous blood sample from the patient and laboratory analysis which both add time to the diagnostic process. In addition, 24 hour laboratory testing may not be available at all locations or with all pre-hospital evaluations. Recent technological advances have made d-dimer point of care (POC) testing available. The recent development of point of care testing may assist with increasing d-dimer testing availability as well as decreasing the time to obtain results (Riley et al., 2016). Since d-dimer levels increase with age, normal value ranges must be adjusted according to age ( $\geq 50$ years old). Age-adjusting d-dimer improves the number of older adults that can be safely excluded as having a PE; however, many false positives still remain, especially among those over the age of 65 years (Crawford et al., 2016; Konstantinides et al., 2014; Righini et al., 2014; Schouten et al., 2014; Woller et al., 2014). Furthermore, d-dimer utility has not been evaluated in pregnant or immediate post-partum patient populations (Solomon \& Greer, 2015).

\section{Radiologic Imaging}

When indicated, radiologic imaging provides the most accurate and reliable PE diagnosis. According to the American College of Physicians (ACP) and European guidelines, radiologic imaging is indicated for patients who do not meet all the PERC criteria, have an intermediate PE clinical probability plus a positive d-dimer, or have a high PE clinical probability (Konstantinides et al., 2014; Raja et al., 2015) (see Figure 
2.1). Radiologic imaging for definitive PE diagnosis can include computed tomography pulmonary angiography (CTPA), pulmonary angiography and/or ventilation to perfusion (V/Q) scanning.

\section{Computed Tomography Pulmonary Angiography (CTPA)}

The CTPA has now replaced the pulmonary angiogram as the accepted gold standard for PE diagnosis (Dogan, de Roos, Geleijins, Huisman, \& Kroft, 2015; Konstantinides et al., 2014; Ma et al., 2017; Raja et al., 2015). CTPA is fast, reliable and easily accessibility in most hospital settings. The PIOPED II study determined the CTPA to be $83 \%$ sensitive and $96 \%$ specific with a $96 \%$ PPV in patients with a high or low clinical probability and 92\% PPV in patients with an intermediate probability Wells score (Stein et al., 2006). Improved technology has made the multi-detector CTPA both sensitive and specific for PE diagnosis and when used in conjunction with other clinical predictors (CDR \& d-dimer), only 5\% of CTPAs are inconclusive (Parikh et al., 2015; van der Hulle, Dronkers, Klok, \& Huisman, 2016). Thus, the clinician can be confident in both the negative and positive CPTA result. It should be noted, however, that obesity and severe tachypnea increase the rate of inconclusive exams to $10 \%$ (Kline \& Kabrhel, 2015). Another useful aspect of this imaging study is that CTPA can provide the clinician with an alternative diagnosis when the scan is negative for PE. For example, in a study by van Es (2013), the CTPA provided an alternative diagnosis for the presenting symptoms in roughly $50 \%$ of the PE negative patients.

CTPA Overuse. The downside to CTPA accessibility is overuse of the technology (Konstantinides et al., 2014; Raja et al., 2015).CTPA overuse leads to unnecessary exposure to imaging related risks as well as increased health care costs. 
Nationally, only about $10 \%$ of CTPA scans are positive for PE, indicating a large number of negative results and potential to eliminate unnecessary exams (Parikh et al., 2015). For example, Parikh and colleagues (2015) found that CTPA was being used on patients with a negative d-dimer which is contrary to current PE guidelines. CTPA overuse also has been noted in settings where clinicians do not routinely utilize a PE CDR or when errors are made in the calculation of the CDR related to education or infrequent use (Newnham, Stone, Summerfield, \& Mustfa, 2013). Indeed, in one single center study where d-dimer and PE CDRs were not regularly utilized, $55 \%$ of patients received an unnecessary CTPA (Perera, Aggarwal, Scott, \& Cocks, 2017). Moreover, CTPA also is being overused in older adult and female patients being evaluated for PE (Bickley et al., 2015; Chen, Gray, Bandiera, MacKinnon, \& Deva, 2015). Adherence to PE practice guidelines has been shown to increase the diagnostic yield of CTPA (Wang et al., 2016). For example, use of a computerized diagnostic decision support system resulted in a $25 \%$ decrease in CTPA testing indicating it may be an effective strategy to help eliminate CTPA overuse (Jiménez et al., 2015).

CTPA Risks. While CTPA provides a definitive diagnosis, it also carries significant risks including radiation exposure, allergic reaction to intravenous (IV) contrast and contrast induced nephrotoxicity (CIN). A CTPA exposes the patient to a significant amount of ionizing radiation that typically ranges between 10 to 20 millisieverts (mSv) with some reports as high as 70mSv (Kline \& Kabrhel, 2015; Lapner $\&$ Kearon, 2013). The current industry standard for occupational radiation exposure is 50mSv/year (Rohner et al., 2013). The proposed 2018 International Commission on Radiologic Protection guideline suggests lowering occupational exposure to 
$<10 \mathrm{mSv} / y e a r$ with an attempt to keep the yearly dose to $2-4 \mathrm{mSv}$ or less. Exposure to $100 \mathrm{mSv}$ has a $1 \%$ increased risk of developing cancer in a lifetime (Rohner et al., 2013). Medical imaging is the primary source of radiation exposure in the United States (Dainiak, 2013; Rohner et al., 2013). Exposure to $>10 \mathrm{mSv}$ is thought to be carcinogenic, leading to an increased risk of leukemia, thyroid and breast cancer (Huppmann, Johnson, \& Javitt, 2010). The risk of radiation exposure varies by tissue and organs. Breast tissue is one of the more sensitive with only $13 \mathrm{mSv}$ of radiation needed to increase the risk of breast cancer (Alkhorayef, Babikir, Alrushoud, Al-Mohammed, \& Sulieman, 2017). Compared to a 2 view mammography, the CTPA exposes women to a 10-20 fold increase in radiation (Alkhorayef et al., 2017; Freeman, 2007). The younger the age at exposure, along with genetic factors, may increase the risk of radiation injury (Huppmann et al., 2010; Schembri, Miller, \& Smart, 2010). Critically ill patients have an even greater risk of exposure as they often undergo repeated CT scans (Rohner et al., 2013). Avoiding unnecessary CTPA is an important strategy to decreased radiation risks.

Contrast is given to enhance the quality of the imaging. Life threatening reactions to IV contrast occur in $0.1 \%$ of the population with an unexpected death rate from the allergic reaction being 1 in 75,000 (Jung et al., 2012). The incidence and severity of allergic reaction in emergency department patients is low $(0.2 \%)$ with $70 \%$ described as mild and $30 \%$ as moderate. The most common allergic reactions are skin or mucosal (92\%) followed by respiratory (22\%) with gastrointestinal being the lowest (4\%). Allergic symptoms typically occur within 5 minutes of contrast injection and include urticaria (61\%), sneezing/rhinorrhea (32\%) and shortness of breath (22\%) (Gottumukkala et al., 2017). Patients with a history of allergic reactions can be pretreated with steroids 
and antihistamines to mitigate development of a reaction; however, pre-treatment usually occurs over several hours. In emergency situations or when allergy history is unknown, clinicians need to be prepared to treat allergic reactions (Gottumukkala et al., 2017).

Contrast induced nephropathy (CIN) is defined as an increase in serum creatinine by $25 \%$ over baseline that occurs 48 to 72 hours following contrast administration. Risk factors for CIN include diabetes mellitus, chronic kidney injury, heart failure, and nephrotoxic medications (Huisman \& Klok, 2015). Administration of normal saline prior to the IV contrast appears to decrease CIN risk in patients with one or more CIN risk factors (Traub et al., 2013). Recent studies suggest that the CIN risk may have been over estimated and is actually lower than previously thought (Kooiman et al., 2012; McDonald et al., 2014). Depending on the baseline glomerular filtration rate, the incidence of CIN ranges from a low of $1 \%$ to a high of $14 \%$ (Alhassan, Sayf, Arsene, \& Krayem, 2016; Kooiman et al., 2012; McDonald et al., 2014). Fortunately, research suggests that only $0.06 \%$ of patients require renal replacement therapy after a CT examination. Although the incidence of CIN is low, unnecessary exposure to IV contrast should, nonetheless, be avoided.

\section{Ventilation to Perfusion Scan (V/Q Scan)}

The V/Q scan has largely been replaced by the more accurate CTPA, however, there remains a role for V/Q scans in patients at increased risk for radiation exposure (pregnancy), contrast allergy, and contrast exposure (renal injury) (Freeman, 2007; Hunsaker, Lu, Goldhaber, \& Rybicki, 2010; Konstantinides et al., 2014; Piovella \& Iosub, 2016; Raja et al., 2015; Solomon \& Greer, 2015). Although the patient is exposed to radiation with the V/Q scan (range 0.28 to $0.9 \mathrm{mSv}$ ), the dose is considerably lower 
than that received from a CTPA (Dogan et al., 2015; Freeman, 2007). Another advantage of the V/Q scan is that it can be performed as the bedside if a patient is too hemodynamically unstable to travel to the diagnostic imaging suite. The V/Q scan requires the patient to breathe radioactive gas followed by the IV injection of a radioactive material which allows the comparison of the areas in the lung that are ventilated to the areas in the lung that have perfusion. A normal V/Q scan can rule out a PE (NPV near 100\%) and a high probability V/Q scan can diagnosis a PE (97\% specific) (Fesmire et al., 2011; Hunsaker et al., 2010; Moores, 2011; Worsley \& Alavi, 1995). Unfortunately, nearly $50 \%$ of the V/Q scans will fall in the grey area of low and intermediate probability scans which are less useful to the clinician (van der Hulle et al., 2016; Worsley \& Alavi, 1995). The low probability V/Q scan has less than $20 \%$ likelihood of the patient having a PE and the intermediate probability V/Q has 20 to $80 \%$ likelihood (Worsley \& Alavi, 1995).

Limitations of the V/Q scan include availability, length of the test, imprecision of the test and exposure to radiation and IV contrast. The V/Q scan can take up to 90 minutes to complete; thus, slowing the diagnosis of PE. Lack of availability of 24/7 radiology staff that can perform and interpret the examination limits the available at some healthcare facilities. The clinician may have to supplement the less precise (low and intermediate probability) V/Q scan results with the other clinical information (e.g. transthoracic echocardiogram, Compression ultra sound) to help determine PE likelihood. Additionally, the V/Q scan is not available in the pre-hospital setting.

\section{Pulmonary Angiogram}


Pulmonary angiogram was long considered the gold standard for PE diagnosis; however, it also has been replaced by CTPA. While excellent for PE diagnosis, the pulmonary angiogram is an invasive procedure that carries significant risks such as central venous cannulation (vessel injury, infection, bleeding) and IV contrast exposure (allergic reactions, nephrotoxicity) (Dogan et al., 2015; Kline \& Kabrhel, 2015). The pulmonary angiogram can be difficult to obtain after business hours as it requires a specialist trained in performing the procedure. Additionally, although the fluoroscopy system adjusts the radiation dose to body size, on average, the radiation exposure is approximately 6 mSv (Zanzonico, Rothenberg, \& Strauss, 2006).

The pulmonary angiogram may be used if treatment such as catheter directed thrombolysis or thrombectomy is planned (Konstantinides et al., 2014). Catheter directed thrombolysis increases the risk of bleeding complications associated with the procedure (Konstantinides et al., 2014), however, there are lower bleeding risks than systemic thrombolysis in a high risk population (Kearon et al., 2016). Pulmonary thromboembolectomy is mainly reserved for the patient with CTEPH versus the acute PE (Kearon et al., 2016). Many health care facilities will not have the skilled team required for catheter directed procedures and therapies (Kearon et al., 2016).

\section{Additional Diagnostic Studies}

\section{Chest X-Ray (CXR)}

The CXR is neither sensitive nor specific for PE but can be useful when excluding other causes of chest pain and dyspnea with findings such as pneumonia or pneumothorax (Sverzellati et al., 2014; Worsley \& Alavi, 1995). The optimum CXR 
study for the PE diagnostic process includes posterior and lateral chest views (Sverzellati et al., 2014). In about $10 \%$ of PE positive patients, the CXR will have a wedge shaped opacity (Hampton Hump) indicative of a pulmonary infarction (Lankeit et al., 2012; Lapner \& Kearon, 2013). Additional CXR findings which might suggest PE presence include atelectasis, cardiomegaly, elevated hemidiaphragm, enlarged major pulmonary artery (Fleischner sign), focal hypovolemia with collapse of vessels (Westermark sign), pleural effusion, and/or pulmonary infiltrates (Sverzellati et al., 2014). Research suggests that senior ( $\geq 10$ years' experience) and junior radiologist's ( $\leq 4$ years' experience) only have fair inter-rater agreement $(\mathrm{k}=0.24$; CI: 0.19-0.29) for determining PE presence or absence based on CXR (Sverzellati et al., 2014). Advantages of the CXR are that it can be performed at the bedside, can determine cause of pulmonary symptoms, and direct further radiologic testing. Limitations of the CXR include its low specificity for PE diagnosis and lack of availability in all healthcare settings, particularly in the pre-hospital arena. Additionally, CXR does expose the patient to a low dose of radiation $(\sim 0.01 \mathrm{mSv})$ (Schembri et al., 2010).

\section{Electrocardiogram}

Since the electrocardiogram (ECG) cannot definitively diagnose PE, an ECG may be ordered in an effort to evaluate nonspecific symptoms such as chest pain or dyspnea. For example, the ECG is often used to rule out acute cardiac ischemia as the cause of dyspnea and/or chest pain (Kline \& Kabrhel, 2015). Advantages of the ECG is that it is noninvasive, easy to perform, easy to interpret and readily available in multiple healthcare settings. 
In most cases of PE, the ECG will be completely normal, especially in patients $<60$ years of age (Co, Elbert, \& Chiganos, 2017). The ECG of a person with a PE may show nonspecific changes such as sinus tachycardia, atrial arrhythmias, right axis deviation and a right bundle branch block (Co et al., 2017). Although classic ECG signs

of RV strain such as S-wave in lead I, Q-wave in lead III, and an inverted T-wave in lead III (S1Q3 T3) can be seen (Hunt \& Bull, 2011), research suggests that only 3\% of PE positive patients have the classic S1Q3T3 findings (Co et al., 2017). Indeed, the most common ECG abnormalities were T wave inversion (34.4\%), T wave flattening (29.5), and sinus tachycardia (27.3\%) (Co et al., 2017). The increased availability of the ECG stored in the EMR allows the clinician to compare current and prior ECGs and more easily identify new ECG changes. That said, the ECG cannot be a primary diagnostic tool for PE due to the nonspecific nature of any ECG changes.

\section{Compression Ultrasonography (CUS)}

Since a proximal DVT carries the greatest risk of PE (Salaun, 2011), a CUS can be useful to identify the presence and location of a DVT (Konstantinides et al., 2014). When a DVT is present, the vein will not compress at the location of the thrombus. CUS has a sensitivity of $90 \%$ and a specificity of about $95 \%$ for symptomatic DVT (Konstantinides et al., 2014). If a patient has had a DVT in the last 12 months, remaining thrombus remnants may limit CUS accuracy in identifying new thrombus (Ageno, Squizzato, Wells, Büller, \& Johnson, 2013; Huisman \& Klok, 2015). If prior CUS images are available, the accuracy of identifying new thrombus is improved (Huisman \& Klok, 2015). Advantages of CUS are that it is an inexpensive, noninvasive option that does not 
involve exposure to IV contrast or radiation for DVT diagnosis; however, it is not currently available in the pre-hospital setting.

Although DVT presence is most certainly a risk factor for PE, it is not diagnostic for a PE. Salaun et al. (2011) found that $50 \%$ of the patients positive for a proximal DVT were also positive for PE. That said, presence of a proximal DVT can guide the clinician to order further testing. For example, CUS can be used in conjunction with CTPA. If the CTPA is negative for PE and the CUS is positive for DVT, patients would qualify for anticoagulation which may prevent a future PE (Beckman, Hooper, Critchley, \& Ortel, 2010; Kearon et al., 2016; Konstantinides et al., 2014). It should be noted, however, that absence of a DVT cannot completely eliminate the possibility of a PE.

\section{Transthoracic Echocardiogram}

PE will rarely be diagnosed by a transthoracic echocardiogram (TTE) (Nazerian et al., 2014). Similar to the ECG, the usefulness of the TTE is in the identification of right ventricular (RV) strain that may be the result of a PE (Konstantinides et al., 2014). TTE can assess for the classic McConnell's Sign, regional RV dysfunction with akinesis of the mid-free wall with normal apical wall motion (McConnell et al., 1996; Weekes et al., 2017). The original study found that the McConnell's sign was $77 \%$ sensitive and a $94 \%$ specific for the diagnosis of acute PE, with a positive predictive value of $71 \%$ and a negative predictive value of $96 \%$ (McConnell et al., 1996). Caution must be exercised, however, since clinical conditions other than PE may cause a McConnell's sign such as intracardiac shunts, pulmonary regurgitation, tricuspid regurgitation and or tricuspid stenosis (Walsh \& Moore, 2015; Weekes et al., 2017). 
A systematic review and meta-analysis of 24 studies determined that signs of right heart strain yielded a positive likelihood ratio of 3.12 compared to a lack of signs for right heart strain having a negative likelihood ratio of 0.57 (Fields et al., 2017). The meta-analysis pooled signs used for right heart stain including 10 discrete assessments (60/60 sign, right ventricular hypokinesis, McConnell's sign, pulmonary arterial hypertension, visualization of an right heart thrombus, right ventricular to left ventricle ratio, right ventricular end-diastolic diameter, abnormal or paradoxical septal motion, tricuspid regurgitation, tricuspid annular plane systolic excursion, and RV systolic pressure) (Fields et al., 2017). Individual analysis of the signs indicated that right heart thrombus was not statistically significant for sensitivity or specificity while both the 60/60 sign and the tricuspid annular plane systolic excursion were only significant for specificity (Fields et al., 2017). While TEE can be useful to further evaluate a clinician's suspicion of PE, clinicians cannot exclude PE based on a normal TTE since not all PE will cause RV strain (Fields et al., 2017; Ma, Yan, Zhou, \& Yuan, 2016; Reissig, Copetti, \& Kroegel, 2011). Furthermore, in the situation of a PE, the TTE can help the clinician to quantify the hemodynamic consequences of a PE, to classify the severity of PE and guide therapy (Weekes et al., 2017). For example, a patient with right ventricular strain and also has a systolic $\mathrm{BP}<90 \mathrm{mmHg}$ is considered to have a massive $\mathrm{PE}$ and meets criteria treatment with thrombolytic therapy (Kearon et al., 2016; Konstantinides et al., 2014; Lapner \& Kearon, 2013; Levin et al., 2015). The TTE also can be used in conjunction with CUS in the critically ill patient who is too unstable to transfer for further diagnostic testing. 


\section{Emerging Diagnostic Tools}

\section{Point of Care Ultrasound (POCUS)}

Point of care ultrasound (POCUS) is becoming more available. Nazerian and colleagues (2017) performed an observational study using a POCUS of the legs and lungs to modify the Wells CDR for either presence/absence of DVT and whether an alternative diagnosis if less likely than PE (Nazerian et al., 2017). The results showed that integrating the POCUS for determining the Wells score improved the sensitivity to $69.6 \%$ and specificity to $88.2 \%$ from $57.6 \%$ and $68.2 \%$, respectively, without the POCUS (Nazerian et al., 2017). Both the presence of DVT and alternative diagnosis aspects of the Wells are subjective. The POCUS added an objective measure, thus, improving the Wells score (Nazerian et al., 2017).

Assessment of multiple organs with POCUS improves the utility over a single organ assessment (Nazerian et al., 2014; Zhu \& Ma, 2017). Skilled clinicians can assess lung, heart and extremity veins to evaluate multiple physiologic effects that may be expected from a PE (Nazerian et al., 2014; Zhu \& Ma, 2017). A study of emergency department patients who met criteria based on CDR and d-dimer for intermediate probability for PE were evaluated with multiple organ POCUS. Seventy four percent of patients that were positive for PE were correctly identified with POCUS; however, 5\% of patients with PE were missed (Nazerian et al., 2014). Of note, the accuracy of residents (80\%; 95\% CI: 74.2 to $85.8 \%)$ was lower compared to senior physicians $(85 \%, 95 \%$ CI: 80.7 to $89.3 \%$ ). The use of goal orientated multi-organ POCUS in conjunction with CDR may increase the pre-test probability of PE and improve the utilization of CTPA to further eliminate unnecessary testing (Zhu \& Ma, 2017). The goal directed multi-organ 
POCUS that does not show any abnormality has a negative predictive value of $95 \%$ for excluding PE (Zhu \& Ma, 2017). An additional role for goal directed multi-organ POCUS can be the hemodynamically unstable patient who is too ill to transport for further diagnostic testing (Zhu \& Ma, 2017). Limitation of POCUS is related to availability of equipment and trained personnel. While availability of POCUS is improving, it may not be available in all healthcare settings.

\section{End-tidal carbon dioxide $\left[\mathrm{EtCO}_{2}\right](\mathrm{Capnography}$}

Another non-invasive tool that has been investigated for its utility in the exclusion of a PE diagnosis is a measurement of end-tidal (exhaled) carbon dioxide, also known as capnography. As discussed earlier, the primary physiologic effect of PE is an increase in AVDS. Increased AVDS impairs $\mathrm{CO}_{2}$ elimination (Figure 2.2). As AVDS increases, alveolar gas from non-perfused alveoli $\left(\mathrm{CO}_{2}\right.$ free gas $)$ mixes with alveolar gas from perfused alveoli (normal concentration of $\mathrm{CO}_{2}$ gas), thus, lowering $\mathrm{EtCO}_{2}$. In the case of $\mathrm{PE}, \mathrm{EtCO}_{2}$ is disproportionally low as compared to arterial $\mathrm{CO}_{2}\left(\mathrm{PaCO}_{2}\right) . \mathrm{EtCO}_{2}$ measurements are useful to assess AVDS changes. Based on the pathophysiology of PE, patients with normal AVDS can be excluded from a PE diagnosis (West \& Luks, 2016). A meta-analysis was completed on $\mathrm{EtCO}_{2}$ studies published between 1990 through 2010. All methods of AVDS assessments with capnography were included but outcomes were not differentiated by AVDS assessment type (Manara, D’hoore, \& Thys, 2013). The 14 pooled studies indicated the sensitivity of an $\mathrm{EtCO}_{2}$ assessment for $\mathrm{PE}$ was $80 \%$ (95\% confidence interval (CI) 76 to $83 \%$ ), specificity of $49 \%$ (95\% CI 45 to $51 \%$ ), a negative likelihood ratio (NLR) .32 (95\% CI .23 to .45) and a positive likelihood ratio (PLR) of 2.43 (95\% CI 1.70-3.46). The NLR in this study shows a stronger likelihood of 
identifying patients without PE compared to the PLR that would identify patients with PE (Sedighi, 2013). The meta-analysis suggests that $\mathrm{EtCO}_{2}$ may be a good additional assessment to use in the PE diagnostic process to help identify low risk patients (pre-test probability less than 10\%) that do not have PE (Manara et al., 2013).

Of the methods for estimating AVDS with $\mathrm{EtCO}_{2}$, the absolute $\mathrm{EtCO}_{2}$ value is the easiest, fastest, and only completely noninvasive method. Five recent studies have utilized absolute $\mathrm{EtCO}_{2}$ measurements in the PE diagnostic process (Fabius, Eijsvogel, van der Lee, Brusse-Keizer, \& de Jongh, 2016; Hemnes et al., 2010; Ramme et al., 2016; Riaz \& Jacob, 2014; Rumpf, Krizmaric, Grmec, \& others, 2009). Four of the five studies found a statistically significant difference between the absolute $\mathrm{EtCO}_{2}$ of patients with and without PE (Hemnes et al., 2010; Ramme et al., 2016; Riaz \& Jacob, 2014; Rumpf et al., 2009). Specifically, patients without $\mathrm{PE}$ had a significantly higher $\mathrm{EtCO}_{2}$ than patients with PE. Unfortunately, to date there is no agreed upon $\mathrm{EtCO}_{2}$ cutoff value that can be used to exclude a patient from the diagnosis of PE. Until additional research has established a consistent cutoff score for $\mathrm{EtCO}_{2}$, it is unlikely to be implemented widely.

\section{Conclusion}

Patients with PE may present with a variety of nonspecific signs and symptoms which overlap with multiple other conditions. PE signs and symptoms range from very mild to devastating severe shock states (Kline \& Kabrhel, 2015). Additionally, on occasion, the patient may be asymptomatic or present with sudden death (Konstantinides et al., 2014). Unfortunately, no individual sign or symptom viewed in isolation has the ability to diagnose or exclude PE (Konstantinides et al., 2014). Thus, making a prompt 
accurate diagnosis of PE often is a clinical challenge. For hemodynamically unstable patients suspected of PE, the diagnostic plan is straightforward; they should forgo any other testing and proceed directly to CTPA, if available (Konstantinides et al., 2014; Raja et al., 2015). For all others, the American College of Physicians (Raja et al., 2015) and the European Society of Cardiology (Konstantinides et al., 2014) provide guidelines and suggest best practices for a step wise approach in the diagnosis of PE. Specifically, the step wise approach for the hemodynamically stable patient includes: probability determination; D-dimer testing; and imaging with CTPA (Konstantinides et al., 2014; Raja et al., 2015). It is the responsibility of the clinician to shepherd patients appropriately along the diagnostic algorithm weighing the risk and benefits of each diagnostic test. Limited research indicates a lack of adherence to the PE diagnostic guidelines. Even with adherence to clinical guidelines, there is still a preponderance of negative CTPAs. Additional research is needed to help identify other diagnostic strategies that can be incorporated into the current PE diagnostic guidelines to further eliminate the need for unnecessary, costly radiology imaging (Table 2.2). 


\section{References}

Ageno, W., Squizzato, A., Wells, P. S., Büller, H. R., \& Johnson, G. (2013). The diagnosis of symptomatic recurrent pulmonary embolism and deep vein thrombosis: guidance from the SSC of the ISTH. Journal of Thrombosis and Haemostasis, 11(8), 1597-1602. https://doi.org/10.1111/jth.12301

Alhassan, S., Sayf, A. A., Arsene, C., \& Krayem, H. (2016). Suboptimal implementation of diagnostic algorithms and overuse of computed tomography-pulmonary angiography in patients with suspected pulmonary embolism. Annals of Thoracic Medicine, 11(4), 254260. https://doi.org/10.4103/1817-1737.191875

Alkhorayef, M., Babikir, E., Alrushoud, A., Al-Mohammed, H., \& Sulieman, A. (2017). Patient radiation biological risk in computed tomography angiography procedure. Saudi Journal of Biological Sciences, 24(2), 235-240. https://doi.org/10.1016/j.sjbs.2016.01.011

Aschermann, M., \& Widimský, J. (2015). Comparison of ESC Guidelines 2008 and 2014 Diagnostic and treatment of acute pulmonary embolism. Cor et Vasa, 57(4), e270-e274. https://doi.org/10.1016/j.crvasa.2015.05.013

Beckman, M. G., Hooper, W. C., Critchley, S. E., \& Ortel, T. L. (2010). Venous thromboembolism: a public health concern. American Journal of Preventive Medicine, 38(4 Suppl), S495-501. https://doi.org/10.1016/j.amepre.2009.12.017

Bickley, D., Lindsey, J., Saint-Hilaire, R., Hussain, A., Vu, M., MacMahon, H., Walter, J. (2015). 678: an analysis of the use of CT pulmonary angiography in young women in an urban emergency department. Critical Care Medicine, 43, 171.

https://doi.org/10.1097/01.ccm.0000474506.44733.8f 
Brenner, D. J. (2012). Radiation and chest CT scans. Chest, 142(3), 549-550. https://doi.org/10.1378/chest.12-0490

Chandra, S., Sarkar, P. K., Chandra, D., Ginsberg, N. E., \& Cohen, R. I. (2013). Finding an alternative diagnosis does not justify increased use of CT-pulmonary angiography. $B M C$ Pulmonary Medicine, 13(1), 1-8. https://doi.org/doi:10.1186/1471-2466-13-9

Chen, Y. A., Gray, B. G., Bandiera, G., MacKinnon, D., \& Deva, D. P. (2015). Variation in the utilization and positivity rates of CT pulmonary angiography among emergency physicians at a tertiary academic emergency department. Emergency Radiology, 22(3), 221-229. https://doi.org/10.1007/s10140-014-1265-6

Chopra, N., Doddamreddy, P., Grewal, \& Kumar. (2012). an elevated D-dimer value: a burden on our patients and hospitals. International Journal of General Medicine, 87-92. https://doi.org/10.2147/IJGM.S25027

Co, I., Eilbert, W., \& Chiganos, T. (2017). New Electrocardiographic Changes in Patients Diagnosed with Pulmonary Embolism. The Journal of Emergency Medicine, 52(3), 280285. https://doi.org/10.1016/j.jemermed.2016.09.009

Crawford, F., Andras, A., Welch, K., Sheares, K., Keeling, D., \& Chappell, F. M. (2016). Ddimer test for excluding the diagnosis of pulmonary embolism. Cochrane Database of Systematic Reviews. https://doi.org/10.1002/14651858.CD010864.pub2

Dainiak, N. (2013). Radiation dose and stochastic risk from exposure to medical imaging. CHEST Journal, 144(5), 1431-1433. https://doi.org/10.1378/chest.13-1064 
Dogan, H., de Roos, A., Geleijins, J., Huisman, M., \& Kroft, L. (2015). The role of computed tomography in the diagnosis of acute and chronic pulmonary embolism. Diagnostic and Interventional Radiology, 21(4), 307-316. https://doi.org/10.5152/dir.2015.14403

Douma, R. A., Mos, I. C., Erkens, P. M., Nizet, T. A., Durian, M. F., Hovens, M. M., ... Huisman, M. V. (2011). Performance of 4 clinical decision rules in the diagnostic management of acute pulmonary embolism: a prospective cohort study. Annals of Internal Medicine, 154(11), 709-718. https://doi.org/10.7326/0003-4819-154-11201106070-00002

Dybowska, M., Tomkowski, W. Z., Kuca, P., Ubysz, R., Jóźwik, A., \& Chmielewski, D. (2015). Analysis of the accuracy of the Wells scale in assessing the probability of lower limb deep vein thrombosis in primary care patients practice. Thrombosis Journal, 13(1), 1-5. https://doi.org/10.1186/s12959-015-0050-4

Fabius, T. M., Eijsvogel, M. M., van der Lee, I., Brusse-Keizer, M. G. J., \& de Jongh, F. H. (2016). Volumetric capnography in the exclusion of pulmonary embolism at the emergency department: a pilot study. Journal of Breath Research, 10(4), 046016. https://doi.org/10.1088/1752-7163/10/4/046016

Fesmire, F. M., Brown, M. D., Espinosa, J. A., Shih, R. D., Silvers, S. M., Wolf, S. J., \& Decker, W. W. (2011). Critical issues in the evaluation and management of adult patients presenting to the emergency department with suspected pulmonary embolism. Annals of Emergency Medicine, 57(6), 628-652.e75.

https://doi.org/10.1016/j.annemergmed.2011.01.020 
Fields, J. M., Davis, J., Girson, L., Au, A., Potts, J., Morgan, C. J., ... Riesenberg, L. A. (2017). Transthoracic echocardiography for diagnosing pulmonary embolism: a systematic review and meta-analysis. Journal of the American Society of Echocardiography, 30(7), 714-723.e4. https://doi.org/10.1016/j.echo.2017.03.004

Freeman, L. M. (2007). Don’t bury the v/q scan: it's as good as multidetector CT angiograms with a lot less radiation exposure. Journal of Nuclear Medicine, 49(1), 5-8. https://doi.org/10.2967/jnumed.107.048066

Gibson NS, Sohne M, Kruip MJ, Tick LW, Gerdes VE, Bossuyt PM, Wells PS, \& Buller HR. (2008). Further validation and simplification of the Wells clinical decision rule in pulmonary embolism. Thrombosis Haemostasis. 99(1):229-34. doi: 10.1160/TH07-050321.

Gottumukkala, R. V., Glover, M., Yun, B. J., Sonis, J. D., Kalra, M. K., Otrakji, A., ... Prabhakar, A. M. (2017). Allergic-like contrast reactions in the ED: Incidence, management, and impact on patient disposition. The American Journal of Emergency Medicine. https://doi.org/10.1016/j.ajem.2017.10.032

Hemnes, A. R., Newman, A. L., Rosenbaum, B., Barrett, T. W., Zhou, C., Rice, T. W., \& Newman, J. H. (2010). Bedside end-tidal CO2 tension as a screening tool to exclude pulmonary embolism. European Respiratory Journal, 35(4), 735-741. https://doi.org/10.1183/09031936.00084709 
Hughes, M. J., Stein, P. D., \& Matta, F. (2014). Silent pulmonary embolism in patients with distal deep venous thrombosis: Systematic review. Thrombosis Research, 134(6), 11821185. https://doi.org/10.1016/j.thromres.2014.09.036

Huisman, M. V., \& Klok, F. A. (2015). Current challenges in diagnostic imaging of venous thromboembolism. Blood, 126(21), 2376-2382. https://doi.org/10.1182/blood-2015-05640979

Hunsaker, A. R., Lu, M. T., Goldhaber, S. Z., \& Rybicki, F. J. (2010). Imaging in acute pulmonary embolism with special clinical scenarios. Circulation: Cardiovascular Imaging, 3(4), 491-500. https://doi.org/10.1161/CIRCIMAGING.109.855981

Hunt, J. M., \& Bull, T. M. (2011). Clinical review of pulmonary embolism: diagnosis, prognosis, and treatment. Medical Clinics of North America, 95(6), 1203-1222. https://doi.org/10.1016/j.mcna.2011.08.003

Huppmann, M. V., Johnson, W. B., \& Javitt, M. C. (2010). Radiation risks from exposure to chest computed tomography. Seminars in Ultrasound, CT and MRI, 3I(1), 14-28. https://doi.org/10.1053/j.sult.2009.09.003

Jiménez, D., Resano, S., Otero, R., Jurkojc, C., Portillo, A. K., Ruiz-Artacho, P., ... Yusen, R. D. (2015). Computerized clinical decision support for suspected PE: Table 1. Thorax, 70(9), 909-911. https://doi.org/10.1136/thoraxjnl-2014-206689

Jung, J.-W., Kang, H.-R., Kim, M.-H., Lee, W., Min, K.-U., Han, M.-H., \& Cho, S.-H. (2012). Immediate hypersensitivity reaction to gadolinium-based MRI contrast media. Radiology, 264(2), 414-422. https://doi.org/10.1148/radiol.12112025 
Kahn, S. R., Houweling, A. H., Granton, J., Rudski, L., Dennie, C., \& Hirsch, A. (2014). Longterm outcomes after pulmonary embolism: current knowledge and future research. Blood Coagulation \& Fibrinolysis, 25(5), 407-415. https://doi.org/10.1097/MBC.0000000000000070

Kelly, A.M., Keijers, G., Klim, S., Graham, C., Craig, S., Kuan, W.S. ... Laribi, S. (2017). An observational study of dyspnea in emergency departments: The Asia, Australia, and New Zealand dyspnea in emergency department study (AANZDEM). Academic Emergency Medicine, 24, 328-336. doi: 10.1111/acem.13118.

Kearon, C., Akl, E. A., Ornelas, J., Blaivas, A., Jimenez, D., Bounameaux, H., .. Moores, C. L. K. (2016). Antithrombotic therapy for VTE disease. Chest, 149(2), 315-352. https://doi.org/10.1016/j.chest.2015.11.026

Kline, J. A., Hogg, M. M., Courtney, D. M., Miller, C. D., Jones, A. E., \& Smithline, H. A. (2012). D-dimer threshold increase with pretest probability unlikely for pulmonary embolism to decrease unnecessary computerized tomographic pulmonary angiography: Elevated D-dimer threshold. Journal of Thrombosis and Haemostasis, 10(4), 572-581. https://doi.org/10.1111/j.1538-7836.2012.04647.x

Kline, J. A., \& Kabrhel, C. (2015). Emergency evaluation for pulmonary embolism, part 2: diagnostic approach. The Journal of Emergency Medicine, 49(1), 104-117. https://doi.org/10.1016/j.jemermed.2014.12.041 
Kline, J. A., Mitchell, A. M., Kabrhel, C., Richman, P. B., \& Courtney, D. M. (2004). Clinical criteria to prevent unnecessary diagnostic testing in emergency department patients with suspected pulmonary embolism. Journal of Thrombosis and Haemostasis, 2(8), 12471255. https://doi.org/10.1111/j.1538-7836.2004.00790.x

Kline, J. A., Peterson, C. E., \& Steuerwald, M. T. (2010). Prospective evaluation of real-time use of the pulmonary embolism rule-out criteria in an academic emergency department: perc. Academic Emergency Medicine, 17(9), 1016-1019. https://doi.org/10.1111/j.15532712.2010.00826.x

Klok, F. A., Dzikowska-Diduch, O., Kostrubiec, M., Vliegen, H. W., Pruszczyk, P., Hasenfuß, G., ... Lankeit, M. (2016). Derivation of a clinical prediction score for chronic thromboembolic pulmonary hypertension after acute pulmonary embolism. Journal of Thrombosis and Haemostasis, 14(1), 121-128. https://doi.org/10.1111/jth.13175

Klok, F.A., Mos, I.C.M. , Nijkeuter, M., Righini, M., Perrier, A., Le Gal, G., \& Huisman, M.V., (2008). Simplification of the revised geneva score for assessing clinical probability of pulmonary embolism.Archives of Internal Medicine, 168(19):2131-2136

Konstantinides, S. V., Torbicki, A., Agnelli, G., Danchin, N., Fitzmaurice, D., Galiè, N., ... Spyropoulos, A. C. (2014). 2014 ESC Guidelines on the diagnosis and management of acute pulmonary embolism. European Heart Journal, 35(43), 3033-3080. https://doi.org/10.1093/eurheartj/ehu283

Kooiman, J., Pasha, S. M., Zondag, W., Sijpkens, Y. W. J., van der Molen, A. J., Huisman, M. V., \& Dekkers, O. M. (2012). Meta-analysis: Serum creatinine changes following 
contrast enhanced CT imaging. European Journal of Radiology, 81(10), 2554-2561. https://doi.org/10.1016/j.ejrad.2011.11.020

Le Gal, G., Righini, M., Roy, P.M., Sanchez, O., Aujesky, D., Bounameaux, H.,\& Perrier, A. (2006). Prediction of pulmonary embolism in the emergency department: The revised geneva score. Annals of Internal Medicine, ;144:165-171.

LaMori, J. C., Shoheiber, O., Mody, S. H., \& Bookhart, B. K. (2015). Inpatient resource use and cost burden of deep vein thrombosis and pulmonary embolism in the United States. Clinical Therapeutics, 37(1), 62-70. https://doi.org/10.1016/j.clinthera.2014.10.024

Lankeit, M., GÃṛmez, V., Wagner, C., Aujesky, D., Recio, Mã., Briongos, S., ... JimÃ Šnez, D. (2012). A strategy combining imaging and laboratory biomarkers in comparison with a simplified clinical score for risk stratification of patients with acute pulmonary embolism. CHEST Journal, 141(4), 916-922.

Lapner, S. T., \& Kearon, C. (2013). Diagnosis and management of pulmonary embolism. BMJ, 346, f757. https://doi.org/10.1136/bmj.f757

Lavorini, F., Di Bello, V., De Rimini, M. L., Lucignani, G., Marconi, L., Palareti, G., ... Pistolesi, M. (2013). Diagnosis and treatment of pulmonary embolism: a multidisciplinary approach. Multidisciplinary Respiratory Medicine, 8(1), 1. https://doi.org/10.1186/2049-6958-8-75

Le Roux, P.-Y., Palard, X., Robin, P., Delluc, A., Abgral, R., Querellou, S., ... Salaun, P.-Y. (2014). Safety of ventilation/perfusion single photon emission computed tomography for 
pulmonary embolism diagnosis. European Journal of Nuclear Medicine and Molecular Imaging, 41(10), 1957-1964. https://doi.org/10.1007/s00259-014-2763-1

Levin, D., Seo, J. B., Kiely, D. G., Hatabu, H., Gefter, W., van Beek, E. J. R., \& Schiebler, M. L. (2015). Triage for suspected acute pulmonary embolism: think before opening Pandora's Box. European Journal of Radiology, 84(6), 1202-1211. https://doi.org/10.1016/j.ejrad.2015.03.023

Lippi, G., Danese, E., Favaloro, E., Montagnana, M., \& Franchini, M. (2015). Diagnostics in venous thromboembolism: from origin to future prospects. Seminars in Thrombosis and Hemostasis, 41(04), 374-381. https://doi.org/10.1055/s-0034-1544003

Lucassen, W., Geersing, G.-J., Erkens, P. M., Reitsma, J. B., Moons, K. G., Büller, H., \& van Weert, H. C. (2011). Clinical decision rules for excluding pulmonary embolism: a metaanalysis. Annals of Internal Medicine, 155(7), 448-460.

Ma, Y., Wang, Y., Liu, D., Ning, Z., An, M., Wu, Q., \& Lin, Y. (2017). A safe strategy to rule out pulmonary embolism: The combination of the Wells score and D-dimer test: One prospective study. Thrombosis Research, 156, 160-162. https://doi.org/10.1016/j.thromres.2017.06.018

Ma, Y., Yan, S., Zhou, L., \& Yuan, D.-T. (2016). Competitive assessments of pulmonary embolism: Noninvasiveness versus the golden standard. Vascular, 24(2), 217-224. https://doi.org/10.1177/1708538115589893 
Manara, A., D’hoore, W., \& Thys, F. (2013). Capnography as a diagnostic tool for pulmonary embolism: a meta-analysis. Annals of Emergency Medicine, 62(6), 584-591. https://doi.org/10.1016/j.annemergmed.2013.04.010

Marshall, P. S., Mathews, K. S., \& Siegel, M. D. (2011). Diagnosis and management of lifethreatening pulmonary embolism. Journal of Intensive Care Medicine, 26(5), 275-294. https://doi.org/10.1177/0885066610392658

McConnell, M. V., Solomon, S. D., Rayan, M., Come, P., Goldhaber, S., \& Lee, R. (1996). Regional right ventricular dysfunction detected by echocardiography in acute pulmonary embolism. American Journal of Cardiology, 78, 469-473. https://doi.org/10.1016/S0002$9149(96) 00339-6$

McDonald, J. S., McDonald, R. J., Carter, R. E., Katzberg, R. W., Kallmes, D. F., \& Williamson, E. E. (2014). Risk of intravenous contrast material-mediated acute kidney injury: a propensity score-matched study stratified by baseline-estimated glomerular filtration rate. Radiology, 271(1), 65-73. https://doi.org/10.1148/radiol.13130775

Montagnana, M., Cervellin, G., Franchini, M., \& Lippi, G. (2011). Pathophysiology, clinics and diagnostics of non-thrombotic pulmonary embolism. Journal of Thrombosis and Thrombolysis, 31(4), 436-444. https://doi.org/10.1007/s11239-010-0519-8

Moores, L. K. (2011). Current approach to the diagnosis of acute nonmassive pulmonary embolism. CHEST Journal, 140(2), 509. https://doi.org/10.1378/chest.10-2468

Nazerian, P., Vanni, S., Volpicelli, G., Gigli, C., Zanobetti, M., Bartolucci, M., ... Grifoni, S. (2014). Accuracy of point-of-care multiorgan ultrasonography for the diagnosis of 
pulmonary embolism. CHEST Journal, 145(5), 950-957. https://doi.org/10.1378/chest.13-1087

Nazerian, P., Volpicelli, G., Gigli, C., Becattini, C., Sferrazza Papa, G. F., Grifoni, S., ... the Ultrasound Wells Study Group. (2017). Diagnostic performance of wells score combined with point-of-care lung and venous ultrasound in suspected pulmonary embolism. Academic Emergency Medicine, 24(3), 270-280. https://doi.org/10.1111/acem.13130

Newnham, M., Stone, H., Summerfield, R., \& Mustfa, N. (2013). Performance of algorithms and pre-test probability scores is often overlooked in the diagnosis of pulmonary embolism. British Medical Journal, 346(mar19 2), f1557-f1557. https://doi.org/10.1136/bmj.f1557

Nordenholz, K. E., Naviaux, N. W., Stegelmeier, K., Haukoos, J. S., Wolf, S. J., McCubbin, T., \& Heard, K. (2007). Pulmonary embolism risk assessment screening tools: the interrater reliability of their criteria. The American Journal of Emergency Medicine, 25(3), 285290. https://doi.org/10.1016/j.ajem.2006.08.016

Omar, H. R., Mirsaeidi, M., Weinstock, M. B., Enten, G., Mangar, D., \& Camporesi, E. M. (2017). Syncope on presentation is a surrogate for submassive and massive acute pulmonary embolism. The American Journal of Emergency Medicine. https://doi.org/10.1016/j.ajem.2017.11.014

Oqab, Z., Ganshorn, H., \& Sheldon, R. (2017). Prevalence of pulmonary embolism in patients presenting with syncope. A systematic review and meta-analysis. The American Journal of Emergency Medicine. https://doi.org/10.1016/j.ajem.2017.09.015 
Parikh, N., Morris, E., Babb, J., Wickstrom, M., McMenamy, J., Sharma, R., ... Kim, D. (2015). MDCT diagnosis of acute pulmonary embolism in the emergent setting. Emergency Radiology, 22(4), 379-384. https://doi.org/10.1007/s10140-014-1290-5

Perelas, A., Dimou, A., Saenz, A., Rhee, J. H., Teerapuncharoen, K., Rowden, A., \& Eiger, G. (2015). CT pulmonary angiography utilization in the emergency department: diagnostic yield and adherence to current guidelines. American Journal of Medical Quality, 30(6), 571-577. https://doi.org/10.1177/1062860614543302

Perera, M., Aggarwal, L., Scott, I. A., \& Cocks, N. (2017). Underuse of risk assessment and overuse of CTPA in patients with suspected pulmonary thromboembolism: Overuse of CTPA in suspected PTE. Internal Medicine Journal. https://doi.org/10.1111/imj.13524

Piovella, F., \& Iosub, D. I. (2016). Acute pulmonary embolism: risk assessment, risk stratification and treatment options: Acute pulmonary embolism. The Clinical Respiratory Journal, 10(5), 545-554. https://doi.org/10.1111/crj.12264

Planquette, B., Ferré, A., Peron, J., Vial-Dupuy, A., Pastre, J., Mourin, G., ... Sanchez, O. (2016). Residual pulmonary vascular obstruction and recurrence after acute pulmonary embolism. A single center cohort study. Thrombosis Research, 148, 70-75. https://doi.org/10.1016/j.thromres.2016.10.030

Puurunen, M. K., Gona, P. N., Larson, M. G., Murabito, J. M., Magnani, J. W., \& O’Donnell, C. J. (2016). Epidemiology of venous thromboembolism in the Framingham Heart Study. Thrombosis Research, 145, 27-33. https://doi.org/10.1016/j.thromres.2016.06.033 
Raja, A. S., Greenberg, J. O., Qaseem, A., Denberg, T. D., Fitterman, N., \& Schuur, J. D. (2015). Evaluation of patients with suspected acute pulmonary embolism: best practice advice from the clinical guidelines committee of the American college of physicians. Annals of Internal Medicine, 163(9), 701. https://doi.org/10.7326/M14-1772

Ramme, A. J., Iturrate, E., Dweck, E., Steiger, D. J., Hutzler, L. H., Fang, Y., ... Sigmund, A. E. (2016). End tidal carbon dioxide as a screening tool for computed tomography angiogram in postoperative orthopaedic patients suspected of pulmonary embolism. The Journal of Arthroplasty, 31(10), 2348-2352. https://doi.org/10.1016/j.arth.2016.03.033

Reissig, A., Copetti, R., \& Kroegel, C. (2011). Current role of emergency ultrasound of the chest. Critical Care Medicine, 39(4), 839-845. https://doi.org/10.1097/CCM.0b013e318206d6b8

Riaz, I., \& Jacob, B. (2014). Pulmonary embolism in Bradford, UK: role of end-tidal CO2 as a screening tool. Clinical Medicine, 14(2), 128-133. https://doi.org/10.7861/clinmedicine.14-2-128

Righini, M., Van Es, J., Den Exter, P. L., Roy, P.-M., Verschuren, F., Ghuysen, A., ... Le Gal, G. (2014). Age-adjusted d-dimer cutoff levels to rule out pulmonary embolism: the adjust-pe study. JAMA, 311(11), 1117. https://doi.org/10.1001/jama.2014.2135

Riley, R. S., Gilbert, A. R., Dalton, J. B., Pai, S., \& McPherson, R. A. (2016). Widely used types and clinical applications of d-dimer assay. Laboratory Medicine, 47(2), 90-102. https://doi.org/10.1093/labmed/lmw001 
Rohner, D. J., Bennett, S., Samaratunga, C., Jewell, E. S., Smith, J. P., Gaskill-Shipley, M., \& Lisco, S. J. (2013). Cumulative total effective whole-body radiation dose in critically ill patients. Chest, 144(5), 1481-1486. https://doi.org/10.1378/chest.12-2222

Rumpf, T. H., Krizmaric, M., Grmec, S., \& others. (2009). Capnometry in suspected pulmonary embolism with positive D-dimer in the field. Critical Care, 13(6), 1-9. https://doi.org/10.1186/cc8197

Salaun, P.-Y. (2011). Noninvasive diagnosis of pulmonary embolism. CHEST Journal, 139(6), 1294. https://doi.org/10.1378/chest.10-1209

Schembri, G. P., Miller, A. E., \& Smart, R. (2010). Radiation dosimetry and safety issues in the investigation of pulmonary embolism. Seminars in Nuclear Medicine, 40(6), 442-454. https://doi.org/10.1053/j.semnuclmed.2010.07.007

Schouten, H. J., Geersing, G.-J., Oudega, R., van Delden, J. J. M., Moons, K. G. M., \& Koek, H. L. (2014). Accuracy of the wells clinical prediction rule for pulmonary embolism in older ambulatory adults. Journal of the American Geriatrics Society, 62(11), 2136-2141. https://doi.org/10.1111/jgs.13080

Sedighi, I. (2013). Interpretation of diagnostic tests: Likelihood ratio vs predictive value. Iranian Journal of Pediatrics, 23(6), 717.

Sherk, W. M., \& Stojanovska, J. (2017). Role of clinical decision tools in the diagnosis of pulmonary embolism. American Journal of Roentgenology, 208(3), W60-W70. https://doi.org/10.2214/AJR.16.17206 
Singh, B., Mommer, S. K., Erwin, P. J., Mascarenhas, S. S., \& Parsaik, A. K. (2013). Pulmonary embolism rule-out criteria (PERC) in pulmonary embolism—revisited: A systematic review and meta-analysis. Emergency Medicine Journal, 30(9), 701-706. https://doi.org/10.1136/emermed-2012-201730

Smith, S., Geske, J. B., Kathuria, P., Cuttica, M., Schimmel, D. R., Courtney, D. M., ... Wunderink, R. G. (2016). Analysis of national trends in admissions for pulmonary embolism. Chest, 150, 35-45. https://doi.org/10.1016/j.chest.2016.02.638

Smith, S., Geske, J., McNamara, D., Waterer, G., \& Wunderink, R. (2013). Pulmonary embolism trends from 1996 to 2010. Chest, 144(4), 850A. https://doi.org/10.1378/chest.1704692

Solomon, C. G., \& Greer, I. A. (2015). Pregnancy complicated by venous thrombosis. New England Journal of Medicine, 373(6), 540-547. https://doi.org/10.1056/NEJMcp1407434

Stein, P. D., Fowler, S. E., Goodman, L. R., Gottschalk, A., Hales, C. A., Hull, R. D., ... Woodard, P. K. (2006). Multidetector computed tomography for acute pulmonary embolism. New England Journal of Medicine, 354(22), 2317-2327. https://doi.org/10.1056/NEJMoa052367

Stein, P. D., Matta, F., Musani, M. H., \& Diaczok, B. (2010). Silent pulmonary embolism in patients with deep venous thrombosis: a systematic review. The American Journal of Medicine, 123(5), 426-431. https://doi.org/10.1016/j.amjmed.2009.09.037

Stone, J., Hangge, P., Albadawi, H., Wallace, A., Shamoun, F., Knuttien, M. G., ... Oklu, R. (2017). Deep vein thrombosis: pathogenesis, diagnosis, and medical management. 
Cardiovascular Diagnosis and Therapy, 7(S3), S276-S284.

https://doi.org/10.21037/cdt.2017.09.01

Sverzellati, N., De Filippo, M., Quintavalla, M., Randi, G., Franco, F., Cobelli, R., ...

Zompatori, M. (2014). Interobserver reliability of the chest radiograph in pulmonary embolism. Clinical and Applied Thrombosis/Hemostasis, 20(2), 147-151. https://doi.org/10.1177/1076029612458969

Traub, S. J., Mitchell, A. M., Jones, A. E., Tang, A., O’Connor, J., Nelson, T., ... Shapiro, N. I. (2013). N-acetylcysteine plus intravenous fluids versus intravenous fluids alone to prevent contrast-induced nephropathy in emergency computed tomography. Annals of Emergency Medicine, 62(5), 511-520.e25.

https://doi.org/10.1016/j.annemergmed.2013.04.012

Tromeur, C., van der Pol, L. M., Klok, F. A., Couturaud, F., \& Huisman, M. V. (2017). Pitfalls in the diagnostic management of pulmonary embolism in pregnancy. Thrombosis Research, 151, S86-S91. https://doi.org/10.1016/S0049-3848 (17)30075-0

van der Hulle, T., Dronkers, C. E. A., Klok, F. A., \& Huisman, M. V. (2016). Recent developments in the diagnosis and treatment of pulmonary embolism. Journal of Internal Medicine, 279(1), 16-29. https://doi.org/10.1111/joim.12404

van Es, J., Douma, R. A., Schreuder, S. M., Middeldorp, S., Kamphuisen, P. W., Gerdes, V. E., \& Beenen, L. F. (2013). Clinical impact of findings supporting an alternative diagnosis on CT pulmonary angiography in patients with suspected pulmonary embolism. CHEST Journal, 144(6), 1893-1899. 
Walsh, B. M., \& Moore, C. L. (2015). McConnell's sign is not specific for pulmonary embolism: case report and review of the literature. The Journal of Emergency Medicine, 49(3), 301304. https://doi.org/10.1016/j.jemermed.2014.12.089

Wang, R. C., Bent, S., Weber, E., Neilson, J., Smith-Bindman, R., \& Fahimi, J. (2016). The impact of clinical decision rules on computed tomography use and yield for pulmonary embolism: a systematic review and meta-analysis. Annals of Emergency Medicine, 67(6), 693-701.e3. https://doi.org/10.1016/j.annemergmed.2015.11.005

Weekes, A. J., Johnson, A. K., Troha, D., Thacker, G., Chanler-Berat, J., \& Runyon, M. (2017). Prognostic value of right ventricular dysfunction markers for serious adverse events in acute normotensive pulmonary embolism. The Journal of Emergency Medicine, 52(2), 137-150. https://doi.org/10.1016/j.jemermed.2016.09.002

Wells, P. (1998). Use of a clinical model for safe management of patients with suspected pulmonary embolism. Annals of Internal Medicine, 129(12), 997. https://doi.org/10.7326/0003-4819-129-12-199812150-00002

Wells, P., Anderson, D., Bormanis, J., Guy, F., Mitchell, M., Gray, L., ... Lewandowski, B. (1997). Value of assessment of pretest probability of deep-vein thrombosis in clinical management. The Lancet, 350(9094), 1795-1798. https://doi.org/10.1016/S0140$\underline{6736(97) 08140-3}$

Wells, P., Anderson, D., Rodger, M., Ginsberg, J., Kearon, C., Gent, M., ... Hirsh, J. (2000). Derivation of a simple clinical model to categorize patients' probability of pulmonary 
embolism: Increasing the models utility with the simplified d-dimer. Thrombosis and Haemostasis, 83(03), 416-420. doi:10.1055/s-0037-1613830

West, J. B., \& Luks, A. (2016). West's Repiratory Physiology: The Essentials (10th ed.). Philadelphia: Wolters Kluwer.

Wicki, J., Perneger, T., Junod, A., Bounameaux, H., \& Perrier, A. (2000). Assessing clinical probability of pulmonary embolism in the emergency ward: A simple score. Journal of General Internal Medicine, 15(s2), 10-10. doi:10.1046/j.1525-1497.2000.15200-42.x

Woller, S. C., Stevens, S. M., Adams, D. M., Evans, R. S., Lloyd, J. F., Snow, G. L., ... Elliott, C. G. (2014). Assessment of the safety and efficiency of using an age-adjusted d-dimer threshold to exclude suspected pulmonary embolism. Chest, 146(6), 1444-1451. https://doi.org/10.1378/chest.13-2386

Worsley, D. F., \& Alavi, A. (1995). Comprehensive analysis of the results of the pioped study. Journal of Nuclear Medicine, 36(12), 2380-2387.

Yin, F., Wilson, T., Della Fave, A., Larsen, M., Yoon, J., Nugusie, B., ... Chow, R. D. (2012). Inappropriate use of D-dimer assay and pulmonary CT angiography in the evaluation of suspected acute pulmonary embolism. American Journal of Medical Quality, 27(1), 7479. https://doi.org/10.1177/1062860611407907

Youssf, A. R. I., Ismail, M. F. M., ElGhamry, R., \& Reyad, M. R. (2014). Diagnostic accuracy of D-dimer assay in suspected pulmonary embolism patients. Egyptian Journal of Chest Diseases and Tuberculosis, 63(2), 411-417. https://doi.org/10.1016/j.ejcdt.2013.12.015 
Zanzonico, P., Rothenberg, L. N., \& Strauss, H. W. (2006). Radiation exposure of computed tomography and direct intracoronary angiography. Journal of the American College of Cardiology, 47(9), 1846-1849. https://doi.org/10.1016/j.jacc.2005.10.075

Zhu, R., \& Ma, X.-C. (2017). Clinical value of ultrasonography in diagnosis of pulmonary embolism in critically ill patients. Journal of Translational Internal Medicine, 5(4). https://doi.org/10.1515/jtim-2017-0034. 


\section{Figure 2.1. American College of Physicians Best Practice Advice}

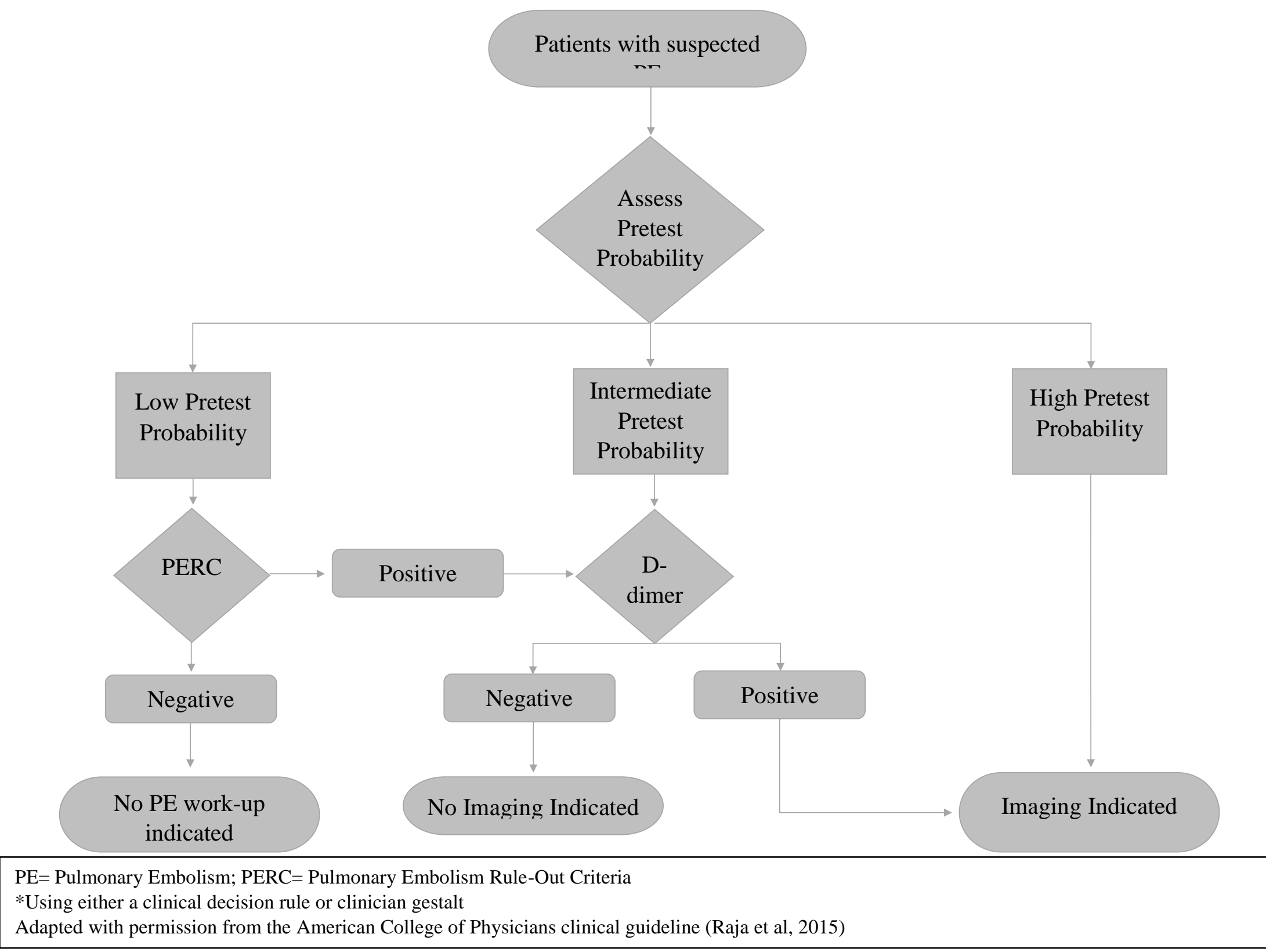


Figure 2.2 Pulmonary Embolism a Iters Alveolar Dead Space and End-Tidal Carbon Dioxide

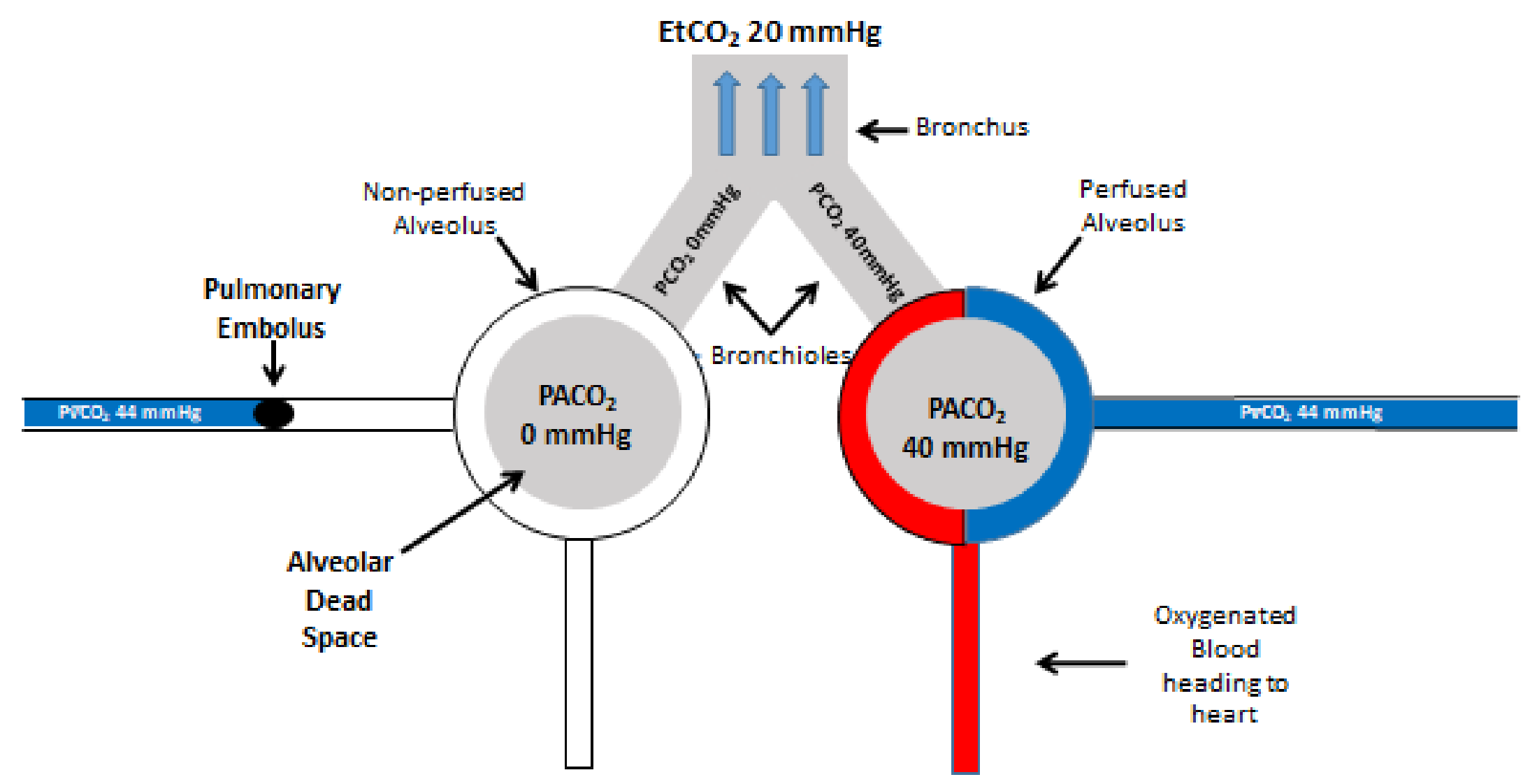

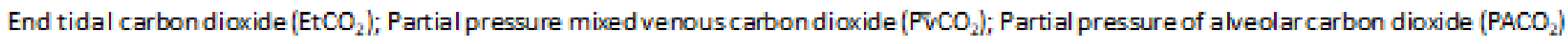




\begin{tabular}{|c|c|c|c|c|c|c|c|}
\hline $\begin{array}{l}\text { Positive } \\
\text { Indicators }\end{array}$ & $\begin{array}{c}\text { Wells/Canadian* } \\
\text { (Point Value) }\end{array}$ & $\begin{array}{l}\text { Modified Wells** } \\
\text { (Point Value) }\end{array}$ & $\begin{array}{l}\text { Simplified Wells** } \\
\text { (Point Value) }\end{array}$ & $\begin{array}{c}\text { Geneva\# } \\
\text { (Point Value) }\end{array}$ & $\begin{array}{l}\text { Revised Geneva\#\# } \\
\text { (Point Value) }\end{array}$ & $\begin{array}{c}\text { Simplified } \\
\text { Revised Genera\#\# } \\
\text { (Point Value) }\end{array}$ & $\begin{array}{l}\text { PERC/Charlotte+ } \\
\text { (Indicators) }\end{array}$ \\
\hline VIE History & $\begin{array}{l}\text { DVT or PE } \\
\text { (1.5 points) }\end{array}$ & $\begin{array}{l}\text { DVT or PE } \\
\text { (1 point) }\end{array}$ & $\begin{array}{l}\text { DVT or PE } \\
\text { (1 point) }\end{array}$ & $\begin{array}{l}\text { DVT or PE } \\
\text { (2 points) }\end{array}$ & $\begin{array}{l}\text { DVT or PE } \\
\text { (3 points) }\end{array}$ & $\begin{array}{l}\text { DVT or PE } \\
\text { (1 point) }\end{array}$ & $\begin{array}{l}\text { DVT or PE } \\
\text { (Presence) }\end{array}$ \\
\hline $\begin{array}{c}\text { Recent } \\
\text { Immobilization, } \\
\text { Surgery, or Trauma }\end{array}$ & $\begin{array}{l}\text { Immobilization } \\
\geq 3 \text { days or } \\
\text { Surgery } \leq 4 \text { weeks } \\
\text { (1.5 points) }\end{array}$ & $\begin{array}{l}\text { Immobilization } \\
\geq 3 \text { days or } \\
\text { Surgery } \leq 4 \text { weelks } \\
\text { (1 point) }\end{array}$ & $\begin{array}{l}\text { Immobilization } \\
\geq 3 \text { dizs or } \\
\text { Surgery } \leq 4 \text { weeks } \\
\text { (1 point) }\end{array}$ & $\begin{array}{l}\text { Surgery } \leq 4 \text { weeks } \\
\text { (3 points) }\end{array}$ & $\begin{array}{l}\text { Surgery or LE Fracture } \\
\leq 1 \text { month } \\
\text { (2 points) }\end{array}$ & $\begin{array}{l}\text { Surgery or LE Fracture } \\
\leq 1 \text { month } \\
\text { (1 point) }\end{array}$ & $\begin{array}{c}\text { Recent surgery or Trauma } \leq 1 \\
\text { month } \\
\text { (Presence) }\end{array}$ \\
\hline Heart Rate & $\begin{array}{l}>100 \\
(1.5 \text { points })\end{array}$ & $\begin{array}{r}>100 \\
(1 \text { point })\end{array}$ & $\begin{array}{r}>100 \\
\text { (1 point) }\end{array}$ & $\begin{array}{c}>100 \\
(1 \text { point })\end{array}$ & $\begin{array}{l}75-94 \text { (3 points) } \\
\geq 95(5 \text { points })\end{array}$ & $\begin{array}{l}75-94 \text { (1 point) } \\
\geq 95 \text { (1 point) }\end{array}$ & $\begin{array}{c}>100 \\
\text { (Presencos) }\end{array}$ \\
\hline $\begin{array}{c}\text { DVT } \\
\text { Clinical Signs \& } \\
\text { Symptoms }\end{array}$ & $\begin{array}{c}\text { Minimum of leg swelling \& pain } \\
\text { with palpation of deepveins } \\
\text { ( } 3 \text { points) }\end{array}$ & $\begin{array}{l}\text { Minimum of leg swelling \& pain with } \\
\text { palpation ofdeep veins } \\
\text { (2 points) }\end{array}$ & $\begin{array}{l}\text { Minimum of leg swelling \& pain } \\
\text { with palpation of deepveirs } \\
\text { (1 } \text { point) }\end{array}$ & Not assessed & $\begin{array}{l}\text { Unilateral LE pain } \\
\text { (3 points) } \\
\text { Pain on limb palpation- } \\
\text { unilateral edema } \\
\text { (4 points) }\end{array}$ & $\begin{array}{c}\text { Unilateral LE pain } \\
\text { (1 point) } \\
\text { Pain on limb palpation- } \\
\text { unilateral edema } \\
\text { (1 point) }\end{array}$ & $\begin{array}{l}\text { Unilateral leg swelling } \\
\text { (Presence) }\end{array}$ \\
\hline Age in Years & Not asseased & Not assessed & Not assessed & $\begin{array}{l}60-79 \text { (1 point) } \\
\geq 80 \text { ( } 2 \text { points) }\end{array}$ & $\begin{array}{l}265 \\
\text { (1 point) }\end{array}$ & $\begin{array}{l}\geq 65 \\
\text { (1 point) }\end{array}$ & $\begin{array}{c}\geq 50 \\
\text { (Presence) }\end{array}$ \\
\hline Hemoptysis & $\begin{array}{c}\text { Hemoptysis } \\
\text { (1 point) }\end{array}$ & $\begin{array}{l}\text { Hemoptysis } \\
\text { (1 point) }\end{array}$ & $\begin{array}{c}\text { Hemoptyis } \\
\text { (1 point) }\end{array}$ & Not assessed & $\begin{array}{l}\text { Hemoptysis } \\
\text { (2 points) }\end{array}$ & $\begin{array}{c}\text { Hemoptysis } \\
\text { (1 point) }\end{array}$ & $\begin{array}{l}\text { Hemoptysis } \\
\text { (Presences) }\end{array}$ \\
\hline Malignancy & $\begin{array}{l}\text { Treated Malifnanncy } \\
\leq 6 \text { months or Palliative } \\
\text { (1 point) }\end{array}$ & $\begin{array}{l}\text { Treated Malignancy } \\
\leq 6 \text { months or } P \text { alliative } \\
\text { (1 point) }\end{array}$ & $\begin{array}{l}\text { Treated Malignancy } \\
\leq 6 \text { months oo Palliative } \\
\text { (1 point) }\end{array}$ & Not assessed & $\begin{array}{l}\text { Active malignancy or } \\
\text { cured } 1 \text { year } \\
\text { (2 points) }\end{array}$ & $\begin{array}{l}\text { Active maliennancy or } \\
\text { cured } 1 \text { year } \\
\text { (1 point) }\end{array}$ & Not asseased \\
\hline Oxygenation & Not assessed & Not assessed & Not assessed & $\begin{array}{c}\mathrm{PaO}_{2} \text { by RA } \mathrm{RABG} \\
<49 \text { (4 points) } \\
49-59 \text { (3 points) } \\
60-71 \text { (2 points) } \\
72-82 \text { (1 point) }\end{array}$ & Not assessed & Not assessed & $\begin{array}{l}\mathrm{SaO}_{2}<95 \% \\
\text { (on Room Air) } \\
\text { (Presence) }\end{array}$ \\
\hline Ventilation & Not assessed & Not assessed & Not assessed & $\begin{array}{c}\mathrm{P}_{2} \mathrm{CO} \text {, by RA AABG } \\
<36(2 \text { points) } \\
36-39 \text { (1 point) }\end{array}$ & Not assessed & Not assessed & Not assessed \\
\hline $\begin{array}{l}\text { PE Clinical } \\
\text { Suspicion }\end{array}$ & $\begin{array}{l}\text { Alternative diagnosis } \\
\text { less likely than PE } \\
\text { (3 points) }\end{array}$ & $\begin{array}{l}\text { Alternative diagnosis less likely than PE } \\
\qquad(2 \text { points) }\end{array}$ & $\begin{array}{l}\text { Alternative diagnosis less likely } \\
\text { than } \mathrm{PE} \\
\text { (1 point) }\end{array}$ & Not assessed & Not asseased & Not assessed & Not assessed \\
\hline Diagnostic Testing & Not asseased & Not asseased & Not assessed & $\begin{array}{c}\text { Chest } x-\text { ray } \\
\text { Platelikike atalactatisis } \\
\text { (1 point) } \\
\text { Elevated hemidiaghragm } \\
\text { (1 point) }\end{array}$ & Not asseased & Not asseased & Not assessed \\
\hline Medications & Not asseased & Not asseased & Not asseased & Not asseased & Not asseased & Not assessed & $\begin{array}{c}\text { Hormone use } \\
\text { (Presences) }\end{array}$ \\
\hline $\begin{array}{c}\text { Range of Scores } \\
\text { \& } \\
\text { Interpretation of } \\
\text { Clinical Probability }\end{array}$ & $\begin{array}{c}\text { Range } 0-12.5 \\
\text { 3. Level (Original)Scoring } \\
>6 \text { High } \\
\text { 2-6 Moderate } \\
\text { 2Low } \\
\text { 2Level (Well144) Scoring } \\
>4 \text { PE Likely } \\
\text { S4 PE Unlikely }\end{array}$ & $\begin{array}{l}\text { Range } 0-9 \\
>2 \text { PE Likely } \\
\leq 2 \text { PE Unilikely }\end{array}$ & $\begin{array}{l}\text { Range } 0-7 \\
>1 \text { PE Likely } \\
\leq 1 \text { PE Untikely }\end{array}$ & $\begin{array}{c}\text { Range 0-16 } \\
29 \text { High } \\
\text { 5-8 Intermediate } \\
0-4 \text { Low }\end{array}$ & $\begin{array}{c}\text { Range } 0-22 \\
\geq 11 \text { High } \\
4-10 \text { Intermediate } \\
0-3 \text { Low }\end{array}$ & $\begin{array}{c}\text { Range } 0-9 \\
\frac{3 \text { Level Scoring }}{5 \geq \text { High }} \\
\text { 2-4 Intermediate } \\
0-1 \text { Low } \\
\frac{2 \text { Level scoring }}{3 \geq \text { PELikely }} \\
0-2 \text { PE Unlikely }\end{array}$ & $\begin{array}{c}\text { Absence of all indicators rules } \\
\text { out PE in a low probability } \\
\text { group }\end{array}$ \\
\hline
\end{tabular}




\begin{tabular}{|c|c|c|}
\hline $\begin{array}{c}\text { Tests } \\
\end{array}$ & Advantages & Disadvantages \\
\hline \multicolumn{3}{|c|}{$\begin{array}{l}\text { Preliminary Testing: Used early in diagnostic process to determine if further testing } \\
\text { is necessary }\end{array}$} \\
\hline $\begin{array}{l}\text { PE Clinical Diagnostic Rules } \\
\text { (CDR) }\end{array}$ & $\begin{array}{l}\text { Guide clinician as to low, } \\
\text { moderate or high probability } \\
\text { for PE } \\
\text { Easy } \\
\text { Quick } \\
\text { Can eliminate unnecessary } \\
\text { tests } \\
\text { Requires minimal education } \\
\text { Best results obtained with } \\
\text { CDR standardization \& } \\
\text { integration into EMR }\end{array}$ & $\begin{array}{l}\text { Pisa CDR requires dedicated web site } \\
\text { for calculation and interpretation } \\
\text { Wells DVT CDR may be confused } \\
\text { with Wells PE CDR } \\
\text { PERC not validated for inpatient use } \\
\text { Inconsistent use leads to less reliable } \\
\text { results }\end{array}$ \\
\hline D-dimer & $\begin{array}{l}\text { Highly sensitive for presence } \\
\text { of clot } \\
\text { Negative result can help rule } \\
\text { out PE } \\
\text { Widely available in hospitals } \\
\text { May be available as a point of } \\
\text { care test (POCT) } \\
\text { Use with CDR may help } \\
\text { determine need for further } \\
\text { testing }\end{array}$ & $\begin{array}{l}\text { Requires venipuncture } \\
\text { Time delay for results, if not POCT } \\
\text { May not be available in all pre- } \\
\text { hospital settings } \\
\text { Low specificity } \\
\text { Positive result not specific for PE } \\
\text { May not be useful in hospitalized } \\
\text { patient population }\end{array}$ \\
\hline
\end{tabular}

\begin{tabular}{|c|c|c|}
\hline Tests & Advantages & Disadvantages \\
\hline \multicolumn{3}{|c|}{ Definitive Testing: Used for final diagnosis } \\
\hline $\begin{array}{l}\text { Computed Tomography } \\
\text { Pulmonary Angiogram (CTPA) }\end{array}$ & $\begin{array}{l}\text { Preferred test for definitive PE } \\
\text { diagnosis } \\
\text { Widely available in hospital } \\
\text { setting } \\
\text { Quick results }(\sim 10 \text { minutes for } \\
\text { to complete test }) \\
\text { May identify other causes of } \\
\text { patient signs and symptoms } \\
\text { Lower fetal radiation exposure }\end{array}$ & $\begin{array}{l}\text { Requires clinician skilled in } \\
\text { performing \& interpreting results } \\
5 \% \text { of results are indeterminate } \\
\text { Not available in pre hospital settings } \\
\text { Not available as a bedside procedure } \\
\text { Requires IV access able to withstand } \\
\text { a power injection }(5-10 \mathrm{ml} / \mathrm{second}) \\
\text { Moderate Radiation }(10-20 \\
\text { millisieverts }\{\mathrm{mSv}\}) \\
\text { High breast radiation exposure } \\
\text { Potential for renal injury and/or } \\
\text { allergic reaction from IV contrast } \\
\text { exposure }\end{array}$ \\
\hline
\end{tabular}

Ventilation to Perfusion (V/Q) Scan
Able to determine presence of PE (high probability scan) or absence of PE (normal scan) Good option if CPTA is contraindicated Widely available in hospital
Requires a trained clinician to perform procedure $\&$ interpret results Not available in pre hospital settings Requires IV access Longer time to complete testing ( 90 minutes) 
setting

Able to be completed at the bedside

No contrast risk

Lower radiation exposure to breast tissue
Definitive diagnosis Can provide therapeutic intervention (catheter directed thrombolysis or thrombectomy) along with diagnostic testing
Minimal Radiation exposure (.28-0.9 mSv)

Higher fetal radiation exposure than CTPA

Harder to interpret results with underlying lung pathology Most scans categorized as low or intermediate probability preventing definitive diagnosis

Requires a physician and team skilled in performing test

Not available at all hospitals

Not available as a bedside procedure Not available in prehospital settings

Delay in obtaining procedure, especially during off hour shifts Invasive procedure

Risk of infection, bleeding, nerve and blood vessel damage Moderated radiation $(6 \mathrm{mSv})$

Potential for renal injury and/or allergic reaction from IV contrast exposure

\begin{tabular}{|c|c|c|}
\hline Tests & Advantages & Disadvantages \\
\hline \multicolumn{3}{|c|}{$\begin{array}{l}\text { Adjunct Testing: Adds information to help interpret results of other tests and further refines ris } \\
\text { assessment }\end{array}$} \\
\hline Electrocardiogram (ECG) & $\begin{array}{l}\text { Easy to obtain } \\
\text { Noninvasive } \\
\text { Can be completed at the } \\
\text { bedside } \\
\text { Immediate results } \\
\text { Widely available including } \\
\text { pre- hospital settings } \\
\text { Can detect right ventricular } \\
\text { (RV) strain } \\
\text { May provide alternative } \\
\text { explanation for signs and } \\
\text { symptoms }\end{array}$ & Not specific for PE \\
\hline $\begin{array}{l}\text { Transthoracic Echocardiogram } \\
\text { (TTE) }\end{array}$ & $\begin{array}{l}\text { Noninvasive } \\
\text { Can be completed at the } \\
\text { bedside } \\
\text { Immediate results } \\
\text { May be available in pre } \\
\text { hospital settings } \\
\text { Can determine presence and } \\
\text { severity of RV strain } \\
\text { May provide alternative } \\
\text { explanation for signs and } \\
\text { symptoms }\end{array}$ & $\begin{array}{l}\text { Requires a trained clinician to } \\
\text { perform \& interpret results } \\
\text { Imaging capability limited in } \\
\text { morbidly obese individuals } \\
\text { Not specific for PE }\end{array}$ \\
\hline
\end{tabular}


Chest X-ray (CXR)

Compression ultrasonography (CUS)
Noninvasive

Can be completed at the bedside

Results immediately available

Widely available including pre hospital settings

Can determine if pulmonary

infarct is present

May provide alternative

explanation for signs and symptoms

Noninvasive

Can be completed at the bedside

Results immediately available

Widely available in hospital

setting and some pre hospital

settings

Able to determine presence and location of venous thrombus, if present
Requires a trained clinician to perform \& interpret results May not be available in some pre hospital settings

Exposure to minimum radiation (0.1 $\mathrm{mSv}$ )

Potential delay related to interpretation by radiologist Not specific for PE

Requires a trained clinician to perform \& interpret results Delay in interpretation May not be available in pre hospital settings

Imaging capability limited in morbidly obese individuals Not specific for PE

May not be able to determine new from pre-existing thrombus

\begin{tabular}{|c|c|c|}
\hline Tests & Advantages & Disadvantages \\
\hline \multicolumn{3}{|l|}{ Emerging Diagnostic Tools } \\
\hline $\begin{array}{l}\text { End-tidal Carbon Dioxide } \\
\left(\mathrm{EtCO}_{2}\right)(\text { Capnography) }\end{array}$ & $\begin{array}{l}\text { Easy to preform } \\
\text { Immediate results - for } \\
\text { absolute EtCO } \\
\text { Widely available in hospital } \\
\text { and pre hospital settings } \\
\text { Can determine presence or } \\
\text { absence of alveolar dead space } \\
\text { (AVDS) } \\
\text { May be used with other } \\
\text { assessments to guide further } \\
\text { testing (positive D-dimer) } \\
\text { Potential to eliminate } \\
\text { unnecessary CTPA in some } \\
\text { patients }\end{array}$ & $\begin{array}{l}\text { Increased AVDS not specific for PE } \\
\text { Some capnography methods require } \\
\text { an ABG \& mathematical calculations } \\
\text { (Alveolar dead space calculation and } \\
\text { EtCO }_{2} \text { Gradient) } \\
\text { Delay in results if ABG is needed } \\
\text { Lack of established cut off value for } \\
\text { PE exclusion }\end{array}$ \\
\hline $\begin{array}{l}\text { Goal directed Point of Care } \\
\text { Ultrasound (POCUS) }\end{array}$ & $\begin{array}{l}\text { Improves accuracy of CDR by } \\
\text { providing objective } \\
\text { measurements of DVT and/or } \\
\text { other diagnosis likely } \\
\text { Potentially available in all } \\
\text { settings including pre hospital } \\
\text { Multiple organ testing } \\
\text { increases accuracy }\end{array}$ & $\begin{array}{l}\text { Lack of trained clinicians } \\
\text { Lack of specific protocols } \\
\text { May not be specific for PE }\end{array}$ \\
\hline
\end{tabular}

Electronic Medical Record (EMR); Intravenous (IV); Deep Venous Thrombosis (DVT) 


\section{CHAPTER THREE:}

\section{METHODS}

\section{Design}

This study used a single center prospective, descriptive, comparative design comparing the end-tidal carbon dioxide $\left(\mathrm{EtCO}_{2}\right)$ value to the results of a computed tomography pulmonary angiogram (CTPA) in hospitalized patients undergoing evaluation for pulmonary embolism (PE). The dependent variable was presence or absence of PE as determined by CTPA. The predictor variable was the absolute $\mathrm{EtCO}_{2}$ value measured in millimeters of mercury $(\mathrm{mmHg})$. The decision for CTPA was determined independently by the participant's clinician, not by the principal investigator (PI).

\section{Setting and Sample}

The study was conducted in a large urban Midwestern, tertiary care, academic medical center with 17,000 CTPAs performed in 2015 (Green, A., personal communication, July 11, 2016). Participants were a convenience sample of hospitalized English-speaking adults $\geq 18$ years old who were legally competent to provide consent or who had a legal representative available for consent, current use of $\mathrm{EtCO}_{2}$ with mechanical ventilation, able to tolerate the nasal cannula $\mathrm{EtCO}_{2}$, and undergoing a CTPA for the possibility of having an acute PE. Potential participants with the following conditions or treatments were excluded: hemodynamic instability (systolic blood pressure $(\mathrm{BP})<90 \mathrm{mmHg}$ ), patients on high flow Optiflow oxygen, tracheotomy nonmechanically ventilated patients, pregnancy, and initiation of thrombolytic therapy (tissue 
plasminogen activator) prior to $\mathrm{EtCO}_{2}$ assessment (Hemnes et al., 2010; Raja et al., 2015) and prisoners.

Sample Size Determination. Sample size was based on recent publications reporting sensitivity and specificity of $\mathrm{EtCO}_{2}$ in the PE diagnostic process (Fabius, Eijsvogel, van der Lee, Brusse-Keizer, \& de Jongh, 2016; Hemnes et al., 2010; Ramme et al., 2016; Rumpf, Krizmaric, Grmec, \& others, 2009). A sample of 112 (with an estimated PE prevalence of $16 \%$ which would include 18 PE positive subjects) was determined to be sufficient to achieve $80 \%$ power to detect a change in sensitivity from 0.5 to 0.8 using a two-sided binomial test and $>99 \%$ power to detect a change in specificity from 0.5 to 0.9 using a two-sided binomial test. The significance level was preset at 0.05 .

\section{Instruments and Methods}

Clinical Decision Rule. A PE clinical decision rule (CDR) was not incorporated into the electronic medical record (EMR) as part of a clinical decision support system for PE diagnosis at the study facility. The study facility encourages providers in the Emergency Department to use of the Wells criteria CDR when ordering a CTPA; however, this is not part of the inpatient practice. If a CDR was documented in the EMR, the PI recorded the CDR score. For the purposes of this study, if no CDR was recorded, a Wells score, Wells4 score, and a simplified Wells score were calculated by the PI based on data available in the EMR. Information required to calculate any Wells score was: history of venous thromboembolism (VTE), recent surgery/trauma ( $\leq 4$ weeks), heart rate $(\mathrm{HR})>100$, suspected deep vein thrombosis (DVT), hemoptysis, treated malignancy $(<6$ months) or palliative care, immobilization (> 3 days), and alternative diagnosis less likely 
(Lucassen et al., 2011). The pulmonary embolism rule-out criteria (PERC) also was not used at the study facility. The PI evaluated the PERC from information available in the EMR. The information required for the PERC was: age, heart rate, pulse oximetry, unilateral leg swelling, hemoptysis, recent surgery or trauma ( $\leq 4$ weeks), prior DVT or PE, and hormone use (Kline \& Kabrhel, 2015; Raja et al., 2015).

CTPA. The $11 \mathrm{CT}$ scanners used for the diagnosis of PE were made by Siemens Medical (40 Liberty Boulevard, Malvern, PA 19355). The models were: Sensation 64 (n=3); Definition 128 AS (n=4); Definition 64 AS (n=1); FLASH (n=1); Sensation Open $(n=1)$; and Definition Edge $(n=1)$. The CTPA process was completed with a multi slice spiral scan. The participant was asked to hold their breath, there was an 8 to 10 second delay before the first timed bolus ( $100 \mathrm{ml}$ of Optiray IV contrast injected at $4 \mathrm{ml} / \mathrm{second})$ control scan which triggers when the Hounsfield Unit reached 100 (A. Klepner, personal communication, July 15, 2017). The CPTA results were read by a radiology fellow then confirmed by a radiology attending for the final results. The radiologist was blinded to the $\mathrm{EtCO}_{2}$ results. For this study, the final CTPA report was used for the diagnosis of PE. No additional review of the images was performed by the PI.

D-dimer. The D-dimer laboratory test required a blood sample to be obtained (blue-top (3.2\% sodium citrate) tube) from the participant and sent to the laboratory for analysis. No POC D-dimer testing was performed at the study facility. The quantitative D-dimer was performed by an agglutination method on an IL-Tops coagulation instrument made by Instrumentation Laboratory (180 Hartwell Rd, Bedford, MA 01730). The D-dimer value was abstracted from the EMR if available. The PI performed an age adjusted calculation for D-dimer for participants $\geq 50$ years old since the facility 
laboratory does not report and age adjusted value. A normal D-dimer was $<500$ nanograms/ml. No D-dimer testing was ordered by the PI.

EtCO$_{2}$. The Medtronic single patient use FilterLine ${ }^{\circledR}$ etCO $_{2}$ sampling line for non-intubated patients was placed on the patient unless already in place. The reading was obtained by attaching the microstream sampling line to the Capnostream ${ }^{\mathrm{TM}} 20 \mathrm{p}$ patient monitor (Medtronic, 710 Medtronic Parkway, Minneapolis, MN 55432). Accuracy, as published per the manufacturer, of the $\mathrm{EtCO}_{2}$ is $\pm 2 \mathrm{mmHg}$ for $\mathrm{EtCO}_{2}$ values between 0$38 \mathrm{mmHg}$, and $\pm 5 \%+0.08 \%$ for every $1 \mathrm{mmHg}$ above $38 \mathrm{mmHg}$ (Medtronic, 2017). All monitors were evaluated by the biomedical department of the hospital prior to use. Annual biomedical evaluation was completed per hospital and manufacture guidelines.

\section{Procedures and Protocol}

Recruitment and Informed Consent. Staff in the radiology department were instructed about the study purpose and requested to notify the PI of all adults who had an order for CT to rule out PE. The PI was notified of a new order for CTPA for diagnosis of PE though the ordering process in the radiology department. Potential participants or their legal representative were informed of the study, asked to participate, and face-toface or phone consent was obtained. A copy of the consent was given to the participant or legal representative (see Appendix C).

Participant Demographic and Clinical Variables. Additional demographic and clinical variables were obtained from the EMR and documented on an investigator developed data collection sheet (see Appendix D). Variables collected included: age, sex, race, medical co-morbidities, vital signs (BP, HR, respiratory rate (RR), pulse oximetry $\left(\mathrm{SpO}_{2}\right)$ ), payer status (Medicare, Medicaid, dual, self-pay, private ), PERC, PE 
CDR (type and value), D-dimer (age-adjusted value if pertinent ( $\geq 50$ years old) \& absolute), conditions that would have made D-dimer positive (recent ( $\leq 4$ weeks) surgery or trauma, invasive procedure, post-partum $\leq 4$ weeks, sepsis/inflammation, rheumatoid arthritis, seizures, African American race, age > 50, smoking, immobility, DVT, coronary artery disease), arterial blood gas and documented PE risk factors (cancer, post- surgery < 1 month, immobility, post- partum $<1$ month, prior DVT/PE, smoking status), serum creatinine pre CT scan and at 72 hours post CT scan. Data from the EMR was obtained for the documented reason the primary clinician ordered the PE protocol CT scan. The PI did not obtain any demographic or clinical variables data from participant nor clinician interview.

\section{Adherence to 2015 American College of Physicians Best Practice Advice for}

\section{the Evaluation of Patients with Suspected Acute Pulmonary Embolism. The}

American College of Physicians (ACP) established six best practice advice (BPA) for the diagnosis of PE (Raja et al., 2015). Compliance with best practice guidelines were evaluated as a whole as well as each separate best practice advice was detailed to allow for evaluation of where breakdown of best practice occurred. The best practice advice is as follows:

1. Clinicians should use validated clinical prediction rules to estimate pretest probability in patients in whom acute PE is being considered.

2. Clinicians should not obtain D-dimer measurements or imaging studies in patients with a low pretest probability of PE and who meet all Pulmonary Embolism Rule-Out Criteria.

3. Clinicians should obtain a high sensitivity D-dimer measurement as the initial diagnostic test in patients who have 
an intermediate pretest probability of PE or in patients with low pretest probability of PE who do not meet all Pulmonary Embolism Rule-Out Criteria. Clinicians should not use imaging studies as the initial test in patients who have a low or intermediate pretest probability of PE.

4. Clinicians should use age-adjusted D-dimer thresholds (age $\times 10$ $\mathrm{ng} / \mathrm{mL}$ rather than a generic $500 \mathrm{ng} / \mathrm{mL}$ ) in patients older than 50 years to determine whether imaging is warranted.

5. Clinicians should not obtain any imaging studies in patients with a D-dimer level below the age adjusted cutoff.

6. Clinicians should obtain imaging with CT pulmonary angiography (CTPA) in patients with high pretest probability of PE. Clinicians should reserve ventilation-perfusion scans for patients who have a contraindication to CTPA or if CTPA is not available. Clinicians should not obtain a D-dimer measurement in patients with a high pretest probability of $\mathrm{PE}$ (Raja et al., 2015, p 701).

EtCO $\mathrm{O}_{2}$ Measurement. Participants had an $\mathrm{EtCO}_{2}$ measured by a PI within 24 hours of the CTPA scan. $\mathrm{EtCO}_{2}$ data were collected in an inpatient hospital room. To minimized pre-analytic variability, participants were either in a seated or supine position. Spontaneously breathing participants had the nasal $\mathrm{EtCO}_{2}$ cannula placed by the PI. If the participant was on oxygen the $\mathrm{EtCO}_{2}$ cannula was set at the same liter flow. The cannula remained in place until a steady state had occurred (approximately 30 seconds to 2 minutes). The $\mathrm{EtCO}_{2}$ value and waveform was displayed on the Capnostream ${ }^{\mathrm{TM}} 20 \mathrm{p}$ patient monitor (Medtronic, Minneapolis, MN). In this setting, all mechanically ventilated participants had continuous $\mathrm{EtCO}_{2}$ with a Philips IntelliVue MP90 (Philips North America Corporation, Andover, MA) patient monitor microstream system as part 
of their routine care. The currently displayed $\mathrm{EtCO}_{2}$ value was recorded. The PI was blinded to the $\mathrm{CT}$ results prior to $\mathrm{EtCO}_{2}$ data collection.

Institutional Cost for Adding $\mathrm{EtCO}_{2}$ to the PE Diagnostic Process. The cost of the $\mathrm{EtCO}_{2}$ disposable nasal cannula was $\$ 13.20$. The cost was calculated for the study patients then extrapolated to institutional cost based on the number of acute PE patients diagnosed annually. Based on the 2015 data available at the study facility, there were 2,361 patients diagnosed with acute PE (Green, A., personal communication July 8, 2016) and 3,321 inpatient Chest CT with contrast were performed in 2017 (Lombardo, J., personal communication, October 10, 2018).

\section{Estimated healthcare cost savings (billable charge) for Avoiding Unnecessary}

CTPA. Charge for CTPA at study facility was $\$ 2,132$ (Lombardo, J., personal communication, October 10, 2018). The number of patients who met the $\mathrm{EtCO}_{2}$ criteria multiplied by the cost of the CTPA was used to calculate the healthcare cost savings. The cost of the $\mathrm{EtCO}_{2}$ disposable multiplied by the number of patients in the study determined the cost addition for adding the $\mathrm{EtCO}_{2}$ assessment.

\section{Data Management and Analysis}

\section{Data Management}

Confidentiality was protected through coding of responses. Individual coded data collection sheets were stored in locked filing cabinet in the PI's locked office, with access limited to members of the research team. Data were double entered into a password protected data base, using a Excel file on the PI's encrypted lap top (Hewlett Packard, HP Inc. Palo Alto, CA) and checked for accuracy prior to data analysis. The two data sets were compared to evaluate for missing or extreme values. In addition box and whisker 
plots were used to check for outliers meeting the \pm 1.5 times the interquartile range. In addition, the electronic data were backed up and stored on an external hard drive that was kept in a locked filing cabinet in the PI's locked office. Analysis was conducted on aggregate de-identified data using the data statistical package IBM SPSS version 24 (IBM Corporation, 1 New Orchard Road, Armonk, New York 10504-1722). Data files were stored in a secure, password protected Box account that was only accessible by the PI, statistician and chair of dissertation committee.

\section{Data Analysis}

Statistical analysis of the data was performed with the support of a statistician and statistical data package (SPSS v 24). General descriptive statistics were used to summarize the data. Continuous variables were reported as a mean and standard deviation and analyzed by an independent t-test. Categorical variables were reported as percentages and analyzed by Chi-squared test or Fisher's exact test in the case of small cell sizes. Statistical procedures used for each research question are described below. Level of significance was preset at $\mathrm{p}<0.05$.

Specific Aim 1: To compare absolute end-tidal carbon dioxide $\left(\mathrm{EtCO}_{2}\right)$ levels in the PE diagnostic process for hemodynamically stable hospitalized adult patients. RQ 1a: What is the level of $\mathrm{EtCO}_{2}$ that provides the best sensitivity and specificity for the exclusion of an acute PE in hemodynamically stable hospitalized adult patients? To address RQ1a, a Receiver Operator Curve (ROC), using the area under the curve data, was used to determine the optimal $\mathrm{EtCO}_{2}$ value to discriminate between patients with and without PE. 
RQ 1b: What is the negative predictive value of an $\mathrm{EtCO}_{2}$ level in hemodynamically stable hospitalized adult patients being evaluated for an acute PE? To address RQ1b, the negative predictive value was calculated using the optimal $\mathrm{EtCO}_{2}$ value that was determined in RQ1a.

Specific Aim 2: To examine the financial impact of the use of $\mathrm{EtCO}_{2}$ during the PE diagnostic process for hemodynamically stable hospitalized adult patients. RQ 2a: What is the estimated institutional cost for adding $\mathrm{EtCO}_{2}$ to the PE diagnostic process in hemodynamically stable hospitalized adult patients? To address RQ2a, the cost of the disposable $\mathrm{EtCO}_{2}$ equipment was calculated by multiplying the cost by the total number of patients included in the study. Annual cost was calculated based on the number of patients that historically receive a CTPA to rule out PE.

RQ 2b: What is the estimated cost savings (billable charge) for avoiding unnecessary computed tomography pulmonary angiogram for hemodynamically stable hospitalized adult patients without a PE? To address RQ2b, the estimated charge determined based on average billable charge for CTPA (\$2132) at the study institution. Annual estimated cost savings (billable charge) was calculated by multiplying the cost of CTPA by the number of patients that met criteria $\left(\mathrm{EtCO}_{2}\right.$ cut off value) minus the cost of the $\mathrm{EtCO}_{2}$ disposable $(\$ 13.20)$. We calculated the number of people that needed to be tested using $\mathrm{EtCO}_{2}$ to prevent someone who did need the CTPA from getting it.

Specific Aim 3: To examine adherence to the current best practice regarding the PE diagnostic process for hemodynamically stable hospitalized adult patients. 
RQ 3a: What is the overall rate of adherence to the 2015 American College of Physicians guidelines for the diagnosis of acute PE in hemodynamically in stable adult hospitalized patients? To address RQ3a, the overall percent of adherence was calculated as the number of yes answers divided by the total number of subjects. Each standard was evaluated separately to determine overall percentage of adherence.

RQ 3b: What is the rate of adherence to the 2015 American College of Physicians six individual best practice advice statements for the diagnosis of acute PE? To address RQ3b, The statements were individually examined and assigned a yes/no. The rate of adherence was calculated taking the sum of yeses divided by the total number of participants for each individual statement. Each individual statement was evaluated to see if there is one statement that is causing the overall adherence in RQ3a to be lower.

Specific Aim 4: To quantify the actual and potential harm associated with unnecessary use of CTPA in the PE diagnostic process for hemodynamically stable hospitalized adult patients.

RQ 4a: What is the frequency of allergic reactions for hemodynamically stable hospitalized adult patients undergoing unnecessary CTPA? To address RQ4a, the presence of allergic reaction was assigned a yes/no. The frequency of allergic reactions was determined by dividing the number of yeses divided by the total number of participants undergoing a CTPA.

RQ 4b: What is the frequency of contrast induced nephropathy (CIN) for hemodynamically stable hospitalized adult patients undergoing unnecessary CTPA? To address RQ4b, the presence of CIN was assigned a yes/no. The frequency of CIN will 
be determined by dividing the number of yeses by the total number of participants undergoing a CTPA.

RQ 4c: How much radiation exposure is avoided by eliminating unnecessary CTPA in hemodynamically stable hospitalized adult patients? To address RQ4c, the average $\mathrm{mSv}$ for CTPA, per individual records, for the participants enrolled in the study. The individual amount of $\mathrm{mSv}$ was added for each participant who was PE negative and divide by the total number of patients that are PE negative.

\section{Human Subjects Considerations}

The study was approved by the PI's PhD committee and the Health Sciences Institutional Review Board at the University of Missouri (see Appendix A). The BarnesJewish Hospital research committee conducted an administrative review. (see Appendix B). Additionally, a minor amendment also was approved to allow for additional clinical data abstraction from the EMR and to increase the number of participants (see Appendix A).

Risks

The participants included in this study were hospitalized patients who receiving a CT scan to rule out PE and who were not hemodynamically unstable (systolic BP $<90$ $\mathrm{mmHg}$ ). Inclusion criteria included: English speaking, $\geq 18$ years old, hemodynamic stable (BP> $90 \mathrm{mmHg}$ systolic), able to provide consent or have a legal authorized representative who can provide consent. Vulnerable populations that were excluded from the study were children (adult hospital), prisoners, and pregnant women. Pregnant women were excluded from the study because the ACP guidelines exclude pregnant women from the current best practice advice (Raja et al., 2015). 
This study involved minimal risk to the study participants. The $\mathrm{EtCO}_{2}$ measurement was non-invasive and painless. There were no known emotional, financial, legal, or social risks to subjects participating in this study. There was a small risk of loss of confidentiality. To minimize this risk each participant was assigned a study code number. The study code number was placed on all paper data collection sheets and also used in the electronic database. The PI accessed the minimum necessary information required to complete the study aims from the EMR in real time and record the information on the coded data collection sheet. The patient identifier (medical record) number was used to access the final CTPA results, but it was not included in the electronic data base. Electronic files were maintained only on a password protected computer and paper forms in a locked office in a locked cabinet.

\section{Adequacy of Protections against Risk}

Patients who were alert and oriented and meet inclusion criteria were approached by the PI to introduce the study and to obtain permission for the study. Discussion about the study was done privately in the patient's hospital room. Since some eligible patients who did not have capacity to consent the legally authorized representatives (LARs) were approached by the PI for enrollment as per IRB approval from University of Missouri and Washington University. The PI described the details of the study to the patient or LAR, including the potential risks and benefits and provided them with the consent form. All questions were answered prior to obtaining consent. The voluntary nature of the study and ability to withdraw at any time was discussed. There was no coercion or undue influence during the consent process. 
Oversight of the study was provided by the PI, Donna Prentice, members of her $\mathrm{PhD}$ dissertation committee and the biostatistician for the School of Nursing. Donna Prentice assured that informed consent was obtained prior to performing any research procedures, that all subjects meet eligibility criteria, and that the study was conducted according to the IRB-approved research plan. The PI reviewed all enrolled patients and any protocol deviations weekly. All patients in the study were hospitalized under the care of physicians and nurses. This was a measurement study only and not a treatment or intervention study, unforeseen complications were highly unlikely. The PI minimized the risk of breach of protected health information by minimizing the number of people who had access to patient identifiers. The PI stored all clinical data via encrypted computer. De-identified electronic data were also stored on a secure served in Box at the University of Missouri.

\section{Potential Benefits of Research to Human Subjects \& Others}

There were no direct benefits to the study participants. Being in this study may prevent unnecessary radiologic testing for patients suspected of PE in the future. Eliminating unnecessary CTPA can prevent exposure to radiation, IV contrast injury (allergic reactions and contract induced renal injury) and eliminate unnecessary health care cost. Society will benefit in the future if $\mathrm{EtCO}_{2}$ assessment can reduce the number of patients that undergo radiologic testing for PE.

\section{Importance of Knowledge to Be Gained}

The frequency of unnecessary CTPA is rising. The availability, quality and reliability along with fear of overlooking a potential life threatening PE are factors leading to the overuse of CTPA. The result of overuse can place an unnecessary financial 
burden on the healthcare system and unnecessary health risks to the patient. CTPA exposes the patient to the risk of radiation and intravenous contrast (allergic reaction and renal injury). Unnecessary imaging should be avoided while still providing a safe diagnostic process for $\mathrm{PE}$. While $\mathrm{EtCO}_{2}$ is adding an additional test to the diagnostic process of PE, it is lower in cost, non-invasive and provides quick results. Best Practice advice exists from the ACP (Raja et al., 2015) to guide clinicians through the diagnostic process. Evidence is surfacing that shows clinicians often overlook this advice. Understanding which of the advice statements is lacking adherence can provide a foundation to investigate the reasons for nonadherence.

\section{Inclusion of Women, Minorities, \& Children}

Minorities and non-pregnant women were included in the study sample. Pregnant women were excluded because the current best practice advice is not validated in pregnant women and CTPA may not be ordered due to the radiation risk to fetus. Children were not included since the research was conducted at an adult tertiary hospital. No sex/gender or ethnic exclusions exist for this study. 
CHAPTER FOUR:

RESULTS

END-TIDAL CARBON DIOXIDE LEVEL IN THE DIAGNOSIS OF ACUTE PULMONARY EMBOLISM IN HOSPITALIZED ADULT PATIENTS

(Prentice, D., Wipke-Tevis, D. D., De Roche, C. B.) 


\begin{abstract}
Background: Since PE symptoms are nonspecific, definitive diagnosis requires costly confirmatory testing with pulmonary computed tomography (CTPA). CTPA risks include allergic reactions, contrast-induced nephropathy $(\mathrm{CIN})$ and radiation exposure. A low cost, non-invasive diagnostic test, such as $\mathrm{EtCO}_{2}$, is needed to help exclude a PE diagnosis. The purpose of this study was to examine the accuracy, financial impact and harm avoidance of adding $\mathrm{EtCO}_{2}$ to the $\mathrm{PE}$ diagnostic process in hemodynamically stable hospitalized adult patients. Methods: A single center prospective, descriptive, correlational design was utilized to compare $\mathrm{EtCO}_{2}$ values to CTPA scan results. Both the radiologist and investigator were blinded to the $\mathrm{EtCO}_{2}$ or $\mathrm{CT}$ result, respectively.

Results: A total of 111 patients had definitive CT results. PE prevalence was $18.9 \%$ $(\mathrm{n}=21)$. Mean $\pm \mathrm{SD} \mathrm{EtCO}_{2}$ was lower $(28 \pm 7.8 \mathrm{mmHg})$ for $\mathrm{PE}$ positive versus $\mathrm{PE}$ negative $(33 \pm 8.1 \mathrm{mmHg})$ participants $(p=.01)$. The $\mathrm{EtCO}_{2}$ ROC curve had an area of .674 (CI .542 - .805). For PE exclusion, an $\mathrm{EtCO}_{2}$ cut off value of $42 \mathrm{mmHg}$ yielded a sensitivity of $100 \%$, specificity of $12.2 \%$ and a NPV of $100 \%$. This cut off could safely eliminate 11 patients (9.9\%) from receiving unnecessary CTPA scans. Conclusions: Adding an $\mathrm{EtCO}_{2}$ assessment is a low cost, non-invasive step to the PE diagnostic process that lowers the number of people who need to undergo PE protocol CTPA eliminating exposure to radiation and intravenous contrast. Approximately $10 \%$ of PE protocol CTPA scans could be eliminated with an $\mathrm{EtCO}_{2}$ value $\geq 42 \mathrm{mmHg}$. For every 6 patients assessed with $\mathrm{EtCO}_{2}$ we can save 1 from an unnecessary CTPA scan.
\end{abstract}




\section{End-Tidal Carbon Dioxide Level in the Diagnosis of Acute Pulmonary Embolism in Hospitalized Adult Patients}

Pulmonary embolism (PE), the third most frequent cardiovascular disease, carries significant morbidity and mortality (LaMori, Shoheiber, Mody, \& Bookhart, 2015). Given that PE manifestations overlap with several medical conditions, prompt diagnosis can be challenging (Kline \& Kabrhel, 2015). Further, the diagnostic process carries significant potential health risks from radiation and contrast dye exposure (Brenner, 2012; Dainiak, 2013; Kooiman et al., 2012; McDonald et al., 2014; Rohner et al., 2013). Moreover, healthcare costs for definitive PE diagnosis are substantial. For example, in 2010, the billable charge for computed tomography pulmonary angiogram (CTPA) was approximately $\$ 2,000$ (Hemnes et al., 2010). Despite guideline advice for a systematic PE diagnostic process (Raja et al., 2015) with judicious use of imaging, patients at low risk of PE still undergo unnecessary CTPA (Perelas et al., 2015a; Perera, Aggarwal, Scott, \& Cocks, 2017). Indeed, research suggests only $10 \%$ of CTPAs will be positive for PE (Parikh et al., 2015; Perelas et al., 2015b; Perera et al., 2017; van Es et al., 2013). If PE could be accurately, quickly, and safely ruled out with an inexpensive, noninvasive tool, such as end-tidal carbon dioxide, then PE negative patients could be prevented from enduring the additional time, risks, and costs associated with unnecessary CTPA.

Acute PE is one condition that causes a sudden increase in alveolar dead space (AVDS) ventilation, where the alveoli remain ventilated but without perfusion (West \& Luks, 2016). Although AVDS can fluctuate with different disease states, it is minimal in a healthy lung (West \& Luks, 2016). Despite the physiology of AVDS being well established, only recently has bedside clinical assessment of AVDS become practical. 
Specifically, the development of bedside end-tidal $\mathrm{CO}_{2}\left(\mathrm{EtCO}_{2}\right)$ monitoring (i.e. capnography), has made bedside assessment of AVDS possible. $\mathrm{EtCO}_{2}$ equipment is simple to use and easily available in most healthcare settings. The addition of an AVDS assessment with $\mathrm{EtCO}_{2}$ may meet the challenge of an easy, rapid, and low cost assessment to exclude additional patients from overuse of radiologic imaging.

Therefore, the purposes of this study were threefold: 1) to determine what level of $\mathrm{EtCO}_{2}$ provides the best sensitivity, specificity and negative predictive value for the exclusion of an acute PE in hemodynamically stable (systolic blood pressure $(\mathrm{BP}) \geq 90$ $\mathrm{mmHg}$ ) adult hospitalized patients, 2) to examine the financial impact of the use of $\mathrm{EtCO}_{2}$ during the PE diagnostic process, and 3) to quantify the actual and potential harm associated with unnecessary use of CTPA in the PE diagnostic process.

\section{Methods}

A single center, prospective, descriptive, comparative design was utilized to compare the $\mathrm{EtCO}_{2}$ value to the results of a CTPA in hospitalized adults undergoing evaluation for suspected PE. The outcome of interest was PE presence or absence as determined by CTPA. The predictor variable was absolute $\mathrm{EtCO}_{2} \mathrm{mmHg}$. The decision to order CTPA was determined independently by the participants' clinician. This study was conducted in accordance with the amended Declaration of Helsinki. The study protocol was approved by the University of Missouri Institutional Review Board (\#2009689) as well as the Research Review Committee at the study site with waiver of documentation of written consent. Informed consent was obtained from all study participants or their legal authorized representative. 


\section{Setting and Sample}

The study site was a large 1200 bed, tertiary care academic medical center in a major metropolitan area in the Midwest. In 2017, the study facility performed 3,321 annual inpatient Chest CT scans with contrast. The study site was unable to identify the number of inpatient Chest CT scans performed specifically for PE diagnosis (J.

Lombardo, personal communication, October 10, 2018).

Participants were English speaking, hospitalized adult patients, $\geq 18$ years old, who could tolerate an $\mathrm{EtCO}_{2}$ nasal cannula or were current users of $\mathrm{EtCO}_{2}$ on mechanical ventilation, able to provide consent or had a legal authorized representative willing to provide consent, and had a CTPA ordered to rule out PE between December 2017 and March 2018. Potential participants were excluded if they were prisoners or had any of the following treatments or conditions: administration of thrombolysis prior to $\mathrm{EtCO}_{2}$ assessment, hemodynamic instability (systolic BP $<90 \mathrm{mmHg}$ ), non-mechanically ventilated tracheostomy, Optiflow® nasal cannula, or pregnancy. Based on recent research reporting $\mathrm{EtCO}_{2}$ sensitivity and specificity for the PE diagnostic process (Fabius, Eijsvogel, van der Lee, Brusse-Keizer, \& de Jongh, 2016; Hemnes et al., 2010; Ramme et al., 2016; Rumpf, Krizmaric, \& Grmec, \& 2009), a sample of 112 participants (with an estimated PE prevalence of $16 \%$ ) was determined to be sufficient to achieve $80 \%$ power to detect a change in sensitivity from 0.5 to 0.8 using a two-sided binomial test with a significance level of $P<0.05$.

The PI was notified by the radiology ordering system of potential participants. Participants meeting eligibility criteria had the $\mathrm{EtCO}_{2}$ measured within 24 hours of the 
CTPA. To minimized variability, $\mathrm{EtCO}_{2}$ was collected by the PI while participants were either in a seated or supine position in an inpatient hospital room. Spontaneously breathing participants had the $\mathrm{EtCO}_{2}$ nasal cannula placed by the PI. If the participant was receiving oxygen therapy, the $\mathrm{EtCO}_{2}$ cannula was set at the same liter flow. The cannula remained in place until a steady state had occurred (approximately 30 seconds to 2 minutes). The reading was obtained by attaching the microstream sampling line to the Capnostream $^{\mathrm{TM}}$ 20p patient monitor (Medtronic, 710 Medtronic Parkway, Minneapolis, $\mathrm{MN}$ 55432). Per the manufacturer, $\mathrm{EtCO}_{2}$ accuracy is $\pm 2 \mathrm{mmHg}$ for $\mathrm{EtCO}_{2}$ values between $0-38 \mathrm{mmHg}$, and $\pm 5 \%+0.08 \%$ for every $1 \mathrm{mmHg}$ above $38 \mathrm{mmHg}$ (Medtronic, 2017). All monitors were evaluated by the hospital biomedical department prior to use. Annual biomedical evaluation was completed per hospital and manufacture guidelines. At the study site, all mechanically ventilated participants had continuous $\mathrm{EtCO}_{2}$ with a Philips IntelliVue MP90 (Philips North America Corporation, Andover, MA) patient monitor microstream system as part of their routine care. The currently displayed $\mathrm{EtCO}_{2}$ value was recorded. Demographic and clinical variables were obtained from the electronic medical record (EMR). The PI was blinded to the CTPA results until after $\mathrm{EtCO}_{2}$ data collection. The PI did not interview participants or clinicians.

\section{Computed Tomography Pulmonary Angiography}

The 11 CT scanners used for the diagnosis of PE were made by Siemens Medical (40 Liberty Boulevard, Malvern, PA 19355). The CTPA process was completed with a multi slice spiral scan. The participant was asked to hold their breath, there was an 8 to 10 second delay before the first timed bolus $(100 \mathrm{ml}$ of Optiray IV contrast injected at 4ml/second) control scan which triggers when the Hounsfield Unit reached 100 (A. 
Klepner, personal communication, July, 15, 2017). The CTPA results were read by a radiology fellow then confirmed by a radiology attending for the final results. The radiology fellow and attending were blinded to the $\mathrm{EtCO}_{2}$ results. The final CTPA report was used for the diagnosis of PE. No additional review of the images was performed by the PI.

\section{PE Clinical Decision Rule Determination}

The PI calculated a traditional Wells score for each participant based on EMR data. Information needed to calculate the Wells score included: alternative diagnosis less likely, heart rate $>100$, hemoptysis, history of venous thromboembolism (VTE), immobilization ( $>3$ days), recent surgery/trauma ( $\leq 4$ weeks), suspected DVT, and treated malignancy (<6 months) or palliative care (Lucassen et al., 2011; Wells et al., 2000). Given that all participants had to have a CTPA ordered to rule out PE to be eligible for study inclusion, the PI counted the "PE considered the likely diagnosis" for the Wells calculations unless specifically stated otherwise in the EMR.

\section{Data Analysis}

Data were checked for accuracy, missing and extreme values prior to data analysis. In addition box and whisker plots were used to check for outliers meeting the \pm 1.5 times the interquartile range. Analysis was conducted on aggregate de-identified data with the support of a statistician and the use the data statistical package IBM SPSS version 24 (IBM Corporation, 1 New Orchard Road, Armonk, New York 10504-1722). Continuous variables were reported as a mean and standard deviation (SD) and analyzed by an independent t-test. Categorical variables were reported as percentages and analyzed 
by Chi-squared test or Fisher's exact test in the case of small cell sizes. Level of significance was preset at $\mathrm{p}<0.05$. A Receiver Operating Characteristic Curve (i.e. ROC curve), using the area under the curve data, was used to determine the optimal $\mathrm{EtCO}_{2}$ value to discriminate between patients with and without PE. The NPV was calculated using the optimal $\mathrm{EtCO}_{2}$ value. Annual estimated cost savings (billable charge) was calculated by multiplying the cost of the CTPA scan by the number of patients that met criteria $\left(\mathrm{EtCO}_{2}\right.$ cut off value) minus the cost of the $\mathrm{EtCO}_{2}$ disposable $(\$ 13.20)$. The estimated charge determined based on average billable charge for chest $\mathrm{CT}$ with contrast (\$2132.00) at the study institution (Lombardo, J., personal communication, September 6, 2018). We calculated the number of people that needed to be tested using $\mathrm{EtCO}_{2}$ to prevent someone who did need the CT from getting it. The average, per individual records, radiation exposure (millisieverts $(\mathrm{mSv})$ ) was obtained for each CTPA scan for the participants enrolled in the study. The actual and potential harm was quantified by determining the frequency of allergic reactions and contrast induced nephropathy (CIN) in participants undergoing CTPA and the average amount of radiation exposure that is avoided by eliminating unnecessary CTPA for PE.

\section{Results}

A total of 114 hospitalized adults were consented. Three participants were excluded from the analysis: two did not receive a CTPA; one had an indeterminate CTPA result. Thus, data from 111 participants were analyzed. The typical participant was a 59 year old, Caucasian female from a general hospital division. PE prevalence was $18.9 \%$ (21/111). Overall, PE positive and negative participants were fairly similar in terms of demographics, comorbidities, and PE risk factors (Table 4.1). However, a greater 
proportion of PE negative than PE positive participants $(P<0.05)$ had documentation of the following comorbid conditions: chronic obstructive pulmonary disease and hypertension.

\section{Clinical Presentation During PE Diagnostic Process}

Clinical presentation, PE CDR scores, and documented clinician rationale for obtaining the CTPA can be found in Table 4.2. The clinical presentation of the typical participant being evaluated for PE was normotensive (128/74 $\mathrm{mmHg})$ with mild tachycardia (103 bpm), mild tachypnea (20/min), and mild oxygen desaturation (94\%) while receiving supplemental oxygen therapy. PE positive participants had a higher traditional Wells score $(P<0.001)$ and a greater proportion had a positive compression ultrasound (CUS) test of their lower extremity $(P<0.01)$ compared to PE negative participants. Correspondingly, a greater proportion of the PE negative participants had a negative CUS test $(P<0.05)$ compared to PE positive participants.

Clinicians documented a variety of reasons for ordering the CTPA; however, the most common reasons were dyspnea, increasing need oxygen, low oxygen saturation, and sinus tachycardia. When PE positive and negative participants were compared, a greater proportion of PE positive than PE negative participants had electrocardiogram (ECG) changes $(P<0.02)$, syncope $(P<0.01)$ or unilateral leg swelling $(P<0.01)$ as their documented rationale for ordering a CTPA. Although the average time between hospital admission and the CTPA completion was 5.3 days $( \pm 8.1), 50 \%$ of the participants had the CTPA scan completed $\leq 2$ days from the time they were admitted. Time of admission to CTPA completion was not different between PE positive (5.90 \pm 6.57$)$ and PE negative (5.21 \pm 8.47$)$ participants $(P=.726)$. 


\section{$\mathrm{EtCO}_{2}$ Sensitivity, Specificity and Negative Predictive Value}

$\mathrm{EtCO}_{2}$ was significantly lower for PE positive $(28.0 \pm 7.8 \mathrm{mmHg})$ than $\mathrm{PE}$ negative $(33.0 \pm 8.1 \mathrm{mmHg}, P=.01)$ participants. The average time between $\mathrm{EtCO}_{2}$ assessment and the CTPA completion was 3.8 \pm 4.3 hours (before or after the CTPA) with no significant difference noted between PE positive (3.9 \pm 3.5$)$ and PE negative (3.7 $\pm 4.5 ; P=.844)$ participants. The majority (94.6\%) of $\mathrm{EtCO}_{2}$ measurements occurred $\leq 12$ hours before or after CTPA completion. The longest time between the CTPA scan and $\mathrm{EtCO}_{2}$ measurement was 18.5 hours.

The $\mathrm{EtCO}_{2}$ ROC area under the curve (Figure 4.1) was 0.674 (95\% Confidence Interval: $0.542,0.805$ ). For $\mathrm{PE}$ exclusion, $\mathrm{EtCO}_{2}$ value $\geq 42 \mathrm{mmHg}$ yielded $100 \%$ sensitivity, $12.2 \%$ specificity, and $100 \%$ NPV (Table 4.3). This $\mathrm{EtCO}_{2}$ value could have safely eliminated $11(9.9 \%)$ unnecessary CTPAs. We calculated the number needed to treat and found that 6 patients undergoing evaluation for acute PE would need to be assessed with $\mathrm{EtCO}_{2}$ in order to save 1 patient from an unnecessary CTPA scan.

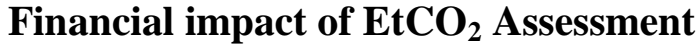

In this study, definitive CTPA scans to rule out PE were completed on 111 hemodynamically stable hospitalized patients during a 3 month time frame which would conservatively annualize to 444 CTPA scans. The use of $\mathrm{EtCO}_{2}$ could potentially eliminate 44 or $10 \%$ of the CTPA scans. Based on the billable charge for the chest CT with contrast at the study site, $\$ 2132$, elimination of $10 \%$ of the scans would save $\$ 93,808$. The cost of supplies to add $\mathrm{EtCO}_{2}$ measures would be about $\$ 5,861$ 
(\$13.20/nasal cannula/patient/assessment x 444 CTPAs). Thus, after deducting the cost of $\mathrm{EtCO}_{2}$ supplies, approximately $\$ 87,947 /$ year in healthcare waste could be avoided.

\section{Actual and Potential Harm Associated with CTPA}

The average radiation exposure for participants receiving a CTPA was $6.2 \pm 3.77$ $\mathrm{mSv}$ which is equivalent to 55 - 64 chest $\mathrm{X}$-rays (2 view chest $\mathrm{X}$-ray/0.1 mSv) (Huppmann, Johnson, \& Javitt, 2010). No significant difference in radiation exposure was found for PE positive $(5.5 \pm 2.5 \mathrm{mSv})$ and PE negative $(6.4 \pm 4.0 \mathrm{mSv})$ participants $(P=.32)$. The overall CIN frequency was $7.2 \%(8 / 92)$. It should be noted, however, that $12.6 \%(14 / 111)$ of participants did not have a follow up serum creatinine ordered at 48 to 72 hours post CTPA scan and could not be evaluated. No significant difference existed between the proportion of PE positive $(9.5 \%)$ and PE negative $(6.7 \%)(P=0.86)$ participants that developed CIN. No participants with CIN required dialysis. No participants had a documented allergic reaction to the CTPA contrast.

\section{Discussion}

This study identified four key findings. First, hemodynamically stable hospitalized adult patients being evaluated for suspected PE with a CTPA were fairly similar in terms of demographics, comorbid conditions, risk factors, and clinical presentation, regardless of actual PE diagnosis. Second, $\mathrm{EtCO}_{2}$ was significantly lower in hemodynamically stable, hospitalized adults who tested positive for PE than those who tested negative for PE. Third, $\mathrm{EtCO}_{2}$ can safely eliminate the diagnosis of PE in a subset of hemodynamically stable hospitalized adults. Specifically, $\mathrm{EtCO}_{2}$ testing could have safely eliminated approximately $10 \%$ of the CTPA scans in these study participants. 
Fourth, adding $\mathrm{EtCO}_{2}$ to the PE diagnostic process could eliminate actual and potential iatrogenic harm as well as healthcare waste associated with overuse of diagnostic imaging for a portion of hospitalized adults evaluated for PE.

\section{Clinical Presentation and the PE Diagnostic Process}

Consistent with the previous PE literature, the demographics, comorbidities, PE risk factor assessment and presenting vital signs did not support the presence of any single specific sign or symptom or even a typical patient profile consistent with a PE diagnosis (Aschermann \& Widimský, 2015; Kline \& Kabrhel, 2015). The lack of definitive signs and symptoms reinforces the need for a structured, evidence-based algorithm to help the guide the clinician in the PE diagnostic process. Clinical decision rules (CDR), such as the Wells score, are one tool to help the clinician coalesce physical signs and symptoms along with medical history to guide the pretest probability for PE (Sherk \& Stojanovska, 2017). Indeed, our data were consistent with prior research that PE positive participants have a significantly higher traditional Wells score than PE negative participants (Lucassen et al., 2011; Posadas-Martínez et al., 2014; Wells, 1998). Unfortunately, use and documentation of a PE CDR is not required at the study site prior to ordering a CTPA which likely contributes to overuse of radiologic imaging in patients being evaluated for PE (Prentice \& Wipke-Tevis, manuscript in preparation).

At this study site, clinicians are, however, required to provide a rationale for ordering the CTPA. Interestingly, the most commonly documented reasons to order the CTPA (dyspnea, increasing need for oxygen, low pulse oximetry and sinus tachycardia) were not significantly different between PE positive and negative participants. In 
contrast, the only clinician documented reasons to order the CTPA that were significantly more common in PE positive than PE negative participants (i.e. ECG changes, unilateral leg swelling, syncope) were cited in $<10 \%$ of participants obtaining a CTPA.

Collectively, these findings reinforce the concept that PE clinical signs and symptoms are vague, overlap with many other conditions and disease processes, and that clinician gestalt is not a sufficient pre-requisite to ordering a CTPA for PE diagnosis in hemodynamically stable adults (Raja et al., 2015). Moreover, given that patients at low risk of PE still undergo unnecessary CTPA even though evidence based PE guidelines exist (Perelas et al., 2015a; Perera, Aggarwal, Scott, \& Cocks, 2017), our findings also suggest that additional, sensitive and specific diagnostic tools, such as $\mathrm{EtCO}_{2}$, are needed to help exclude additional patients from unnecessary CTPA imaging.

\section{Absolute $\mathrm{EtCO}_{2}$, Sensitivity, Specificity, and NPV}

To date, four previous studies have evaluated the usefulness of the time method of absolute $\mathrm{EtCO}_{2}$ assessment in the PE diagnostic process (Hemnes et al., 2010; Ramme et al., 2016; Riaz \& Jacob, 2014; Rumpf, Krizmaric, Grmec et al., 2009). Our findings were consistent with these prior studies in that participants without PE had a significantly higher $\mathrm{EtCO}_{2}$ than participants with PE (Hemnes et al., 2010; Ramme et al., 2016; Riaz \& Jacob, 2014; Rumpf et al., 2009). In order to be a clinically useful diagnostic test for PE exclusion, however, a consistent cutoff value for $\mathrm{EtCO}_{2}$ needs to be established that provides an acceptable and safe sensitivity, specificity, and negative predictive value.

For PE exclusion, our study found that an $\mathrm{EtCO}_{2}$ cut off value of $\geq 42 \mathrm{mmHg}$ yielded a sensitivity of $100 \%$, specificity of $12.2 \%$ and a NPV of $100 \%$. Although the 
aforementioned $\mathrm{EtCO}_{2}$ studies did not find a consistent $\mathrm{EtCO}_{2}$ cutoff value to be utilized for the exclusion of $\mathrm{PE}$ (range $\mathrm{EtCO}_{2} 28-43 \mathrm{mmHg}$ ), our findings are within the range of the previously reported $\mathrm{EtCO}_{2}$ cutoff values (Hemnes et al., 2010; Ramme et al., 2016; Riaz \& Jacob, 2014; Rumpf et al., 2009). Of importance, the findings by Ramme and colleagues (2016), the only other study to examine a sample of exclusively hospitalized patients (postoperative orthopedic surgery patients), were similar to ours in terms of PE prevalence, ROC curve and $\mathrm{EtCO}_{2}$ cutoff value. Specifically, their $\mathrm{EtCO}_{2}$ cutoff value for excluding PE was $\geq 43 \mathrm{mmHg}$ with $100 \%$ sensitivity, $7 \%$ specificity, and NPV of 100\% (Ramme et al., 2016).

In contrast, three earlier studies (Hemnes et al., 2010; Riaz \& Jacob, 2014; Rumpf et al., 2009) found lower $\mathrm{EtCO}_{2}$ cutoff values $(36,32$, and $28 \mathrm{mmHg}$, respectively). We believe that these differences may be due to variations in the study design, sample, and setting. For example, the study by Rumpf and colleagues (2009) included a sample of subjects in the pre-hospital setting and utilized emergency medical services (EMS) personnel to collect the $\mathrm{EtCO}_{2}$. Differences in the $\mathrm{EtCO}_{2}$ cutoff value observed in the Rumpf et al. (2009) study may be related to their sample characteristics such as older mean age and more subjects with syncope episodes. In contrast, the studies by Hemnes et al. (2010) and Riaz and Jacob (2014) included a mixture of both ED and hospitalized patients. Differences in the $\mathrm{EtCO}_{2}$ cutoff value for these two studies may be related to the fact that their sample included patients with a lower frequency of comorbid conditions as well as a greater frequency of patients without any comorbid conditions. Future research with a larger sample size needs to investigate the impact of confounding 
variables such as age and comorbid conditions on the sensitivity, specificity, and NPV of $\mathrm{EtCO}_{2}$ for PE exclusion.

\section{Elimination of Unnecessary Imaging Studies}

Our data suggest an absolute $\mathrm{EtCO}_{2}$ cutoff value of $\geq 42 \mathrm{mmHg}$ could decrease the need for CT scans in up to $10 \%$ of hemodynamically stable hospitalized adult patients suspected of having a PE. In contrast, two of the previous $\mathrm{EtCO}_{2}$ studies (Hemnes et al., 2010; Riaz \& Jacob, 2014) found a much higher number of CT scans that could be eliminated, ranging from 41 to $49 \%$. Again, the findings by the only other inpatient study (Ramme et al., 2016) were similar to our study in that Ramme and colleagues estimated that $5 \%$ of their participants could have avoided a CTPA scan. Differences in the percentage of CTPAs that can be eliminated by using $\mathrm{EtCO}_{2}$ assessment may be related to sample characteristics such as the level of pretest probability determination (i.e. Wells score). Samples that have fewer PE risk factors, fewer comorbidities, and lower pretest probabilities scores, such as was observed in the study by Hemnes and colleagues (2010) and Riaz and Jacob (2014), may have a greater opportunity to avoid unnecessary CTPA.

Additionally, the risk of missing a PE also should be considered when choosing the $\mathrm{EtCO}_{2}$ cutoff score. Our study, in addition to the studies by Ramme et al. (2016) and Riaz and Jacob (2016) had an $\mathrm{EtCO}_{2}$ value with $100 \%$ NPV; meaning no PEs would be missed by use their cutoff value. In contrast, in the studies by Hemnes et al. (2010) and Rumpf et al. (2009) with lower NPVs (96.6\% and 94.2\%, respectively) would result in missing 2 or 3 individuals who actually had a PE. Missing a PE carries significant risk of mortality for the patient and should not be taken lightly. Further study needs to help 
understand the variability in saved CTPA scans to see if the PE risk probability alters the number of scans that will be saved.

\section{Actual and Potential Harm Avoidance}

Our overall CIN rate of $7.2 \%$ is consistent with prior published studies that found CIN impacts 2-7\% of the population that undergo CTPA scans (Alhassan, Sayf, Arsene, \& Krayem, 2016; Kooiman et al., 2012; McDonald et al., 2014; Traub et al., 2013). If $7.2 \%$ of all patients who get a CTPA scan are at risk of getting CIN, then by saving 44 CT scans a year (at this one hospital), you have essentially reduced the risk of 3-4 patients of having CIN (per year at that one hospital). However, by those 44 people not having the CTPA scan, their risk of CIN from a CTPA scan is zero. While most patients who experience $\mathrm{CIN}$ will only experience a brief increase in creatinine, others may experience acute renal injury (Alhassan et al., 2016; Kooiman et al., 2012; McDonald et al., 2014) which, in the worst case scenario, may result in the need for costly dialysis treatments. None of our study participants required dialysis. Nonetheless, given the continuing aging of our society, CIN prevalence associated with contrast is likely to increase since older adults have more risk factors associated with CIN (e.g. chronic kidney disease, dehydration, diabetes mellitus, heart failure, and hypertension). Thus, $\mathrm{EtCO}_{2}$ may be a particularly useful PE diagnostic tool in older adults as it is poses no risk of potential iatrogenic injury and will help eliminate unnecessary CTPA scans in a portion of patients being evaluated for acute PE.

Long-term health risks associated with CTPA scans are due to cumulative radiation exposure (Brenner, 2012; Dainiak, 2013; Le Roux et al., 2014; Parikh et al., 
2015; Rohner et al., 2013; Schembri, Miller, \& Smart, 2010). While the prior studies discussed to the risk of radiation injury and the need to avoid the risk, they did not quantify the amount of exposure (Hemnes et al., 2010; Ramme et al., 2016; Riaz \& Jacob, 2014; Rumpf et al., 2009). Thus, our study was the first $\mathrm{EtCO}_{2}$ study to quantitatively report radiation exposure of patients undergoing CTPA to rule out PE. On average, our study participants undergoing CTPA received a lower dose of radiation (6 $\mathrm{mSv}$ ) than typically reported in the literature (range 10-70 mSv) (Dainiak, 2013; Dogan et al, 2015). Nonetheless, damage to DNA from radiation exposure is cumulative and may result in biologic effects many years following the exposure increasing the risk of cancer (Schembri et al., 2010). Our sample of hemodynamically stable hospitalized adults being evaluated for PE had several comorbid conditions making it likely they may have already undergone previous radiologic testing prior to this hospitalization and likely will undergo future radiologic imaging studies during their lifetime. Avoiding any unnecessary radiologic imaging can lower future risk of developing cancer (Schembri, Miller, \& Smart, 2010).

\section{Financial Impact}

Sparing unnecessary CTPA not only saves the physiologic risks associated with the radiologic imaging but also minimizes healthcare waste. Overuse of testing exposes the patient to harm without benefit. As healthcare providers, we need to ensure the patient gets added value rather than added risk for their dollars. While cost should not be the driving force it, remains an undeniable benefit from avoiding unnecessary testing. We showed a potential of $\$ 2118.80$ per avoidable CTPA. 


\section{Limitations}

The study utilized a convenience sample of hospitalized adults from a single center study which limits the generalizability of the results. Additional multi-site research studies with a larger sample of hospitalized adults are needed to confirm the findings and increase the generalizability. By only using patients who had a CTPA ordered, we may have increased the pretest probability for PE in our sample. Moreover, we did not capture any patients being evaluated for PE that were excluded based on clinician gestalt, PE CDR, PERC, compression ultrasound and/or D-dimer. Future studies need to capture all patients being evaluated for PE to gain a more comprehensive understanding of the potential benefits of $\mathrm{EtCO}_{2}$ assessment in the PE diagnostic process. We relied on documentation in the EMR to determine demographic and clinical variables as well as clinician rationale for ordering the CTPA scan. Therefore, our findings are limited by the accuracy of the clinician documentation. We did not consider the personnel costs associated with implementing $\mathrm{EtCO}_{2}$ assessments on patients being evaluated for PE; thus, our findings may overestimate the financial impact of eliminating unnecessary CTPAs.

\section{Conclusion}

The vague and nonspecific signs and symptoms of patients presenting with PE create a challenge for prompt diagnosis and institution of appropriate PE treatment. Not recognizing and treating PE can lead to death, while unnecessary PE testing and treatment can lead to complications associated with radiologic imaging with intravenous contrast (allergic reaction, acute kidney injury, cumulative radiation exposure risks) and 
anticoagulation (bleeding) as well as healthcare waste. Current practice of pre-test probability assessment (CDR and/or clinician gestalt) with D-dimer testing excludes PE in about $28 \%$ of patients undergoing the PE diagnostic process (van Es, Kraaijpoel, Klok et al., 2017). Given that only 10\% of patients (Parikh et al., 2015; Perelas et al., 2015b; van Es et al., 2013) undergoing the PE diagnostic process test positive for PE using CTPA, an opportunity exists to exclude more patients prior to performing a CTPA.

To this end, we examined the accuracy and harm avoidance of adding $\mathrm{EtCO}_{2}$, an indicator of alveolar dead space, to the PE diagnostic process in a heterogeneous sample of hemodynamically stable hospitalized adult patients. Similar to previous studies using mixed samples of ED and hospitalized patients, we found $\mathrm{EtCO}_{2}$ was significantly lower in PE positive hospitalized adults than PE negative hospitalized adults. Additionally, we found a similar $\mathrm{EtCO}_{2}$ cutoff value to the only other study utilizing all hospitalized adults with a 100\% NPV (Ramme et al., 2016). Collectively, our data suggest that the $\mathrm{EtCO}_{2}$ cutoff value for inpatients may need to be different from $\mathrm{EtCO}_{2}$ cutoff value for ED patients and outpatients. We also found that $\mathrm{EtCO}_{2}$ testing could have safely eliminated approximately $10 \%$ of the CTPA scans in these study participants. Our findings support and extend prior work suggesting that $\mathrm{EtCO}_{2}$ may be a useful additional step in the PE diagnostic process to exclude additional low and/or moderate PE risk patients from a PE diagnosis and unnecessary and costly CTPA imaging. Further study is needed across multiple sites and with larger sample size to validate the $\mathrm{EtCO}_{2}$ cut off value across different patient populations and healthcare settings. 


\section{References}

Alhassan, S., Sayf, A. A., Arsene, C., \& Krayem, H. (2016). Suboptimal implementation of diagnostic algorithms and overuse of computed tomography-pulmonary angiography in patients with suspected pulmonary embolism. Annals of Thoracic Medicine, 11(4), 254-260. doi:10.4103/1817-1737.191875

Aschermann, M., \& Widimský, J. (2015). Comparison of ESC Guidelines 2008 and 2014 - Diagnostic and treatment of acute pulmonary embolism. Cor et Vasa, 57(4), e270-e274. doi:10.1016/j.crvasa.2015.05.013

Brenner, D. J. (2012). Radiation and chest CT scans. Chest, 142(3), 549-550. doi:10.1378/chest.12-0490

Dainiak, N. (2013). Radiation dose and stochastic risk from exposure to medical imaging. CHEST Journal, 144(5), 1431-1433. doi:10.1378/chest.13-1064

Fabius, T. M., Eijsvogel, M. M., van der Lee, I., Brusse-Keizer, M. G. J., \& de Jongh, F. H. (2016). Volumetric capnography in the exclusion of pulmonary embolism at the emergency department: A pilot study. Journal of Breath Research, 10(4), 046016. doi:10.1088/1752-7163/10/4/046016

Hemnes, A. R., Newman, A. L., Rosenbaum, B., Barrett, T. W., Zhou, C., Rice, T. W., \& Newman, J. H. (2010). Bedside end-tidal CO2 tension as a screening tool to exclude pulmonary embolism. European Respiratory Journal, 35(4), 735-741. doi:10.1183/09031936.00084709 
Huppmann, M. V., Johnson, W. B., \& Javitt, M. C. (2010). Radiation risks from exposure to chest computed tomography. Seminars in Ultrasound, CT and MRI, 31(1), 1428. doi:10.1053/j.sult.2009.09.003

Kline, J. A., \& Kabrhel, C. (2015). Emergency evaluation for pulmonary embolism, part 2: Diagnostic approach. The Journal of Emergency Medicine, 49(1), 104-117. doi:10.1016/j.jemermed.2014.12.041

Kooiman, J., Pasha, S. M., Zondag, W., Sijpkens, Y. W. J., van der Molen, A. J., Huisman, M. V., \& Dekkers, O. M. (2012). Meta-analysis: Serum creatinine changes following contrast enhanced CT imaging. European Journal of Radiology, 81(10), 2554-2561. doi:10.1016/j.ejrad.2011.11.020

LaMori, J. C., Shoheiber, O., Mody, S. H., \& Bookhart, B. K. (2015). Inpatient resource use and cost burden of deep vein thrombosis and pulmonary embolism in the United States. Clinical Therapeutics, 37(1), 62-70. doi:10.1016/j.clinthera.2014.10.024

Le Roux, P.-Y., Palard, X., Robin, P., Delluc, A., Abgral, R., Querellou, S., ... Salaun, P.-Y. (2014). Safety of ventilation/perfusion single photon emission computed tomography for pulmonary embolism diagnosis. European Journal of Nuclear Medicine and Molecular Imaging, 41(10), 1957-1964. doi:10.1007/s00259-0142763-1

Lucassen, W., Geersing, G.-J., Erkens, P. M., Reitsma, J. B., Moons, K. G., Büller, H., \& van Weert, H. C. (2011). Clinical decision rules for excluding pulmonary embolism: A meta-analysis. Annals of Internal Medicine, 155(7), 448-460. 
McDonald, J. S., McDonald, R. J., Carter, R. E., Katzberg, R. W., Kallmes, D. F., \& Williamson, E. E. (2014). Risk of intravenous contrast material-mediated acute kidney injury: A propensity score-matched study stratified by baseline-estimated glomerular filtration rate. Radiology, 271(1), 65-73. doi:10.1148/radiol.13130775

Parikh, N., Morris, E., Babb, J., Wickstrom, M., McMenamy, J., Sharma, R., ... Kim, D. (2015). MDCT diagnosis of acute pulmonary embolism in the emergent setting. Emergency Radiology, 22(4), 379-384. doi:10.1007/s10140-014-1290-5

Perelas, A., Dimou, A., Saenz, A., Rhee, J. H., Teerapuncharoen, K., Rowden, A., \& Eiger, G. (2015a). CT pulmonary angiography utilization in the emergency department: diagnostic yield and adherence to current guidelines. American Journal of Medical Quality, 30(6), 571-577. doi:10.1177/1062860614543302

Perelas, A., Dimou, A., Saenz, A., Rhee, J. H., Teerapuncharoen, K., Rowden, A., \& Eiger, G. (2015b). Incidental findings on computed tomography angiography in patients evaluated for pulmonary embolism. Annals of the American Thoracic Society, 12(5), 689-695. doi:10.1513/AnnalsATS.201404-144OC

Perera, M., Aggarwal, L., Scott, I. A., \& Cocks, N. (2017). Underuse of risk assessment and overuse of CTPA in patients with suspected pulmonary thromboembolism: Overuse of CTPA in suspected PTE. Internal Medicine Journal. doi:10.1111/imj.13524

Raja, A. S., Greenberg, J. O., Qaseem, A., Denberg, T. D., Fitterman, N., \& Schuur, J. D. (2015). Evaluation of patients with suspected acute pulmonary embolism: best 
practice advice from the clinical guidelines committee of the American college of physicians. Annals of Internal Medicine, 163(9), 701. doi:10.7326/M14-1772

Ramme, A. J., Iturrate, E., Dweck, E., Steiger, D. J., Hutzler, L. H., Fang, Y., ...

Sigmund, A. E. (2016). End tidal carbon dioxide as a screening tool for computed tomography angiogram in postoperative orthopaedic patients suspected of pulmonary embolism. The Journal of Arthroplasty, 31(10), 2348-2352. doi:10.1016/j.arth.2016.03.033

Riaz, I., \& Jacob, B. (2014). Pulmonary embolism in Bradford, UK: role of end-tidal CO2 as a screening tool. Clinical Medicine, 14(2), 128-133. doi:10.7861/clinmedicine.14-2-128

Rohner, D. J., Bennett, S., Samaratunga, C., Jewell, E. S., Smith, J. P., Gaskill-Shipley, M., \& Lisco, S. J. (2013). Cumulative total effective whole-body radiation dose in critically ill patients. Chest, 144(5), 1481-1486. doi:10.1378/chest.12-2222

Rumpf, T. H., Krizmaric, M., \& Grmec, S. (2009). Capnometry in suspected pulmonary embolism with positive D-dimer in the field. Critical Care, 13(6), 1-9. doi:10.1186/cc8197

Schembri, G. P., Miller, A. E., \& Smart, R. (2010). Radiation dosimetry and safety issues in the investigation of pulmonary embolism. Seminars in Nuclear Medicine, 40(6), 442-454. doi:10.1053/j.semnuclmed.2010.07.007 
Sherk, W. M., \& Stojanovska, J. (2017). Role of clinical decision tools in the diagnosis of pulmonary embolism. American Journal of Roentgenology, 208(3), W60-W70. doi:10.2214/AJR.16.17206

Traub, S. J., Mitchell, A. M., Jones, A. E., Tang, A., O’Connor, J., Nelson, T., ... Shapiro, N. I. (2013). N-acetylcysteine plus intravenous fluids versus intravenous fluids alone to prevent contrast-induced nephropathy in emergency computed tomography. Annals of Emergency Medicine, 62(5), 511-520.e25. doi:10.1016/j.annemergmed.2013.04.012

van Es, J., Douma, R. A., Schreuder, S. M., Middeldorp, S., Kamphuisen, P. W., Gerdes, V. E., \& Beenen, L. F. (2013). Clinical impact of findings supporting an alternative diagnosis on CT pulmonary angiography in patients with suspected pulmonary embolism. CHEST Journal, 144(6), 1893-1899.

Wells, P., Anderson, D., Rodger, M., Ginsberg, J., Kearon, C., Gent, M., ... Hirsh, J. (2000). Derivation of a simple clinical model to categorize patients' probability of pulmonary embolism: Increasing the models utility with the simplified d-dimer. Thrombosis and Haemostasis, 83(03), 416-420. doi:10.1055/s-0037-1613830

West, J. B., \& Luks, A. (2016). West's Repiratory Physiology: The Essentials (10th ed.). Philadelphia: Wolters Kluwer. 
Table 4.1. Participant Demographics, Comorbidities and PE Risk Factors.

\begin{tabular}{|c|c|c|c|c|}
\hline Variable & $\begin{array}{l}\text { Total Sample } \\
\quad(n=111)\end{array}$ & $\begin{array}{l}\text { PE Positive } \\
\quad(n=21)\end{array}$ & $\begin{array}{c}\text { PE } \\
\text { Negative } \\
(n=90)\end{array}$ & $P$ value \\
\hline Mean \pm SD Age $($ yrs $)$ & $59.0 \pm 15.9$ & $59.2 \pm 17.5$ & $59.0 \pm 15.6$ & .953 \\
\hline \multicolumn{5}{|l|}{ Gender (\%) } \\
\hline Male & 36.9 & 38.1 & 36.7 & .903 \\
\hline Female & 63.1 & 61.9 & 63.3 & \\
\hline \multicolumn{5}{|l|}{ Race $(\%)$} \\
\hline Caucasian & 72.1 & 76.2 & 71.1 & .640 \\
\hline African American & 27.9 & 23.8 & 29.9 & \\
\hline \multicolumn{5}{|l|}{ Hospital Unit Type (\%) } \\
\hline General Medical/Surgical & 79.3 & 71.4 & 81.1 & .324 \\
\hline Critical Care & 20.7 & 28.6 & 18.9 & \\
\hline $\begin{array}{l}\text { Mean } \pm \text { SD Hospital LOS } \\
\text { before CTPA }\end{array}$ & $5.3 \pm 8.1$ & $5.9 \pm 6.6$ & $5.2 \pm 8.5$ & .726 \\
\hline \multicolumn{5}{|l|}{ Comorbidities (\%) } \\
\hline None & 2.7 & 0 & 3.3 & .396 \\
\hline Cancer & 41.4 & 47.6 & 40.0 & .523 \\
\hline Cerebral Vascular Accident & 9.0 & 4.8 & 10.0 & .547 \\
\hline Chronic Kidney Disease & 7.2 & 14.3 & 5.6 & .164 \\
\hline COPD & 27.9 & $9.5^{*}$ & 32.2 & .037 \\
\hline Coronary Artery Disease & 16.2 & 14.3 & 16.7 & .790 \\
\hline Diabetes Mellitus & 27.0 & 23.8 & 27.8 & .712 \\
\hline Heart Failure & 14.4 & 19.0 & 13.3 & .502 \\
\hline Hypertension & 60.4 & $38.1 *$ & 65.6 & .037 \\
\hline Pulmonary Hypertension & 7.2 & 4.8 & 7.8 & .630 \\
\hline Sleep Apnea & 18.9 & 14.3 & 20.0 & .547 \\
\hline Other & 55.9 & 47.6 & 57.8 & .399 \\
\hline \multicolumn{5}{|l|}{ PE Risk Factors (\%) } \\
\hline None & 13.5 & 4.8 & 15.6 & .193 \\
\hline Active Cancer & 31.5 & 38.1 & 30.0 & .472 \\
\hline Current DVT & 31.5 & 47.6 & 27.8 & .078 \\
\hline Current HRT & 0 & 0 & 0 & $\mathrm{n} / \mathrm{a}$ \\
\hline Current Smoker & 18.9 & 9.5 & 21.1 & .222 \\
\hline Immobility & 17.3 & 25.0 & 15.6 & .312 \\
\hline Postoperative & 41.4 & 52.4 & 38.9 & .258 \\
\hline Postpartum & 2.7 & 4.8 & 2.2 & .518 \\
\hline
\end{tabular}


Table 4.2. PE Clinical Signs, PE Probability, and Rationale for CTPA.

\begin{tabular}{|c|c|c|c|c|}
\hline Variable & $\begin{array}{l}\text { Total Sample } \\
\quad(n=111)\end{array}$ & $\begin{array}{c}\text { PE } \\
\text { Positive } \\
(n=21)\end{array}$ & $\begin{array}{c}\text { PE } \\
\text { Negative } \\
(\mathbf{n}=90)\end{array}$ & $P$ value \\
\hline Mean \pm SD SBP $(\mathrm{mmHg})$ & $128.2 \pm 22.8$ & $127.5 \pm 23.8$ & $128.3 \pm 22.7$ & .879 \\
\hline Mean \pm SD DBP $(\mathrm{mmHg})$ & $73.9 \pm 15.7$ & $73.6 \pm 17.7$ & $74 \pm 15.3$ & .913 \\
\hline Mean \pm SD HR (bpm) & $103.0 \pm 19.8$ & $101.5 \pm 24.4$ & $103.3 \pm 18.7$ & .708 \\
\hline Mean \pm SD RR $(/ \mathrm{min})$ & $20.0 \pm 4.8$ & $20.0 \pm 4.3$ & $19.9 \pm 4.8$ & .951 \\
\hline $\begin{array}{l}\text { Mean } \pm \text { SD O } \mathrm{O}_{2} \text { Saturation } \\
(\%)\end{array}$ & $94.4 \pm 5.2$ & $92.7 \pm 6.8$ & $94.8 \pm 4.8$ & .114 \\
\hline Supplemental $\mathrm{O}_{2}(\%)$ & 67.6 & 66.7 & 67.8 & .922 \\
\hline $\begin{array}{l}\text { Positive Venous Doppler } \\
(\%)\end{array}$ & $\begin{array}{c}18.9 \\
1.8\end{array}$ & $\begin{array}{c}47.1 \\
0\end{array}$ & $\begin{array}{c}13.8 \\
2.2\end{array}$ & .001 \\
\hline $\begin{array}{l}\text { Upper Extremity } \\
\text { Lower Extremity }\end{array}$ & $\begin{array}{c}37.8 \\
7.2\end{array}$ & $\begin{array}{l}47.6 \\
23.8\end{array}$ & $\begin{array}{c}35.6 \\
3.3\end{array}$ & .003 \\
\hline Both & 53.2 & 28.6 & 58.9 & .012 \\
\hline $\begin{array}{l}\text { Negative Venous Doppler } \\
(\%) \\
\text { Mean } \pm \text { SD Wells Score }^{+}\end{array}$ & $5.47 \pm 1.5$ & $6.48 \pm 1.2 *$ & $5.23 \pm 1.5$ & $<.001$ \\
\hline Rationale for CTPA (\%) & & & & \\
\hline Chest Pain & 17.1 & 19.0 & 16.7 & .794 \\
\hline Cough & 9.0 & 4.8 & 10.0 & .450 \\
\hline Dyspnea & 68.5 & 66.7 & 68.9 & .844 \\
\hline ECG Changes & 1.8 & $9.5^{*}$ & 0 & .003 \\
\hline Hemoptysis & 3.6 & 4.8 & 3.3 & .752 \\
\hline Increasing need for $\mathrm{O}_{2}$ & 46.8 & 47.6 & 46.7 & .937 \\
\hline Low $\mathrm{O}_{2}$ Saturation & 44.1 & 42.9 & 44.4 & .895 \\
\hline New dysrhythmia & 13.5 & 19.0 & 12.2 & .410 \\
\hline Pleuritic Chest Pain & 7.2 & 9.5 & 6.7 & .648 \\
\hline Sinus Tachycardia & 36.0 & 22.5 & 34.4 & .470 \\
\hline Syncope & 1.8 & $9.5^{*}$ & 0 & .003 \\
\hline Unilateral leg swelling & 4.5 & $14.3 *$ & 2.2 & .016 \\
\hline Other & 21.0 & 23.8 & 27.8 & .712 \\
\hline
\end{tabular}

Pulmonary Embolism (PE); Computed tomography pulmonary angiogram (CTPA); Standard Deviation (SD); Systolic Blood Pressure (SBP); Diastolic Blood Pressure (DBP); Heart Rate (HR); Respiratory Rate (RR); Oxygen $\left(\mathrm{O}_{2}\right)$; Electrocardiogram (ECG); * statistical significance 
Table 4.3. Sensitivity/Specificity PPV/NPV for Variable $\mathrm{EtCO}_{2} \mathrm{Cut}$ off

\begin{tabular}{|c|c|c|c|c|c|}
\hline & $\begin{array}{c}\text { Sensitivity } \\
\text { (95\% CI) }\end{array}$ & $\begin{array}{c}\text { Specificity } \\
(95 \% \text { CI })\end{array}$ & $\begin{array}{c}\text { PPV } \\
(95 \% \mathrm{CI})\end{array}$ & $\begin{array}{c}\text { NPV } \\
(95 \% \mathrm{CI})\end{array}$ & $\begin{array}{c}\text { PE missed } \\
(\%)\end{array}$ \\
\hline \multirow[t]{2}{*}{$\mathrm{EtCO}_{2} 28 \mathrm{mmHg}$} & 47.62 & 76.7 & 32.26 & 86.3 & $11(9.9)$ \\
\hline & $(25.71-70.22)$ & $(6.57-84.94)$ & $(20.98-46.07)$ & $(80.42-90.55)$ & \\
\hline \multirow[t]{2}{*}{$\mathrm{EtCO}_{2} 32 \mathrm{mmHg}$} & 66.7 & 60 & 28 & 88.5 & $7(6.3)$ \\
\hline & $(43-85.41)$ & $(49.13-70.19)$ & $(20.77-36.55)$ & $(80.46-93.53)$ & \\
\hline \multirow[t]{2}{*}{$\mathrm{EtCO}_{2} 36 \mathrm{mmHg}$} & 85.7 & 31.1 & 22.5 & 90.32 & $3(2.7)$ \\
\hline & $(63.66-96.95)$ & $(21.77-41.74)$ & $(18.85-26.63)$ & $(75.8-96.53)$ & \\
\hline \multirow[t]{2}{*}{$\mathrm{EtCO}_{2} 41 \mathrm{mmHg}$} & 90.5 & 14.4 & 19.8 & 86.7 & $2(1.8)$ \\
\hline & $(69.62-98.83)$ & $(7.92-23.43)$ & $(17.34-22.5)$ & $(61.32-96.38)$ & \\
\hline \multirow[t]{2}{*}{$\mathrm{EtCO}_{2} 42 \mathrm{mmHg}$} & 100 & 12.2 & 21 & 100 & $0(0)$ \\
\hline & $(83.89-100)$ & $(6.26-20.82)$ & $(19.75-22.31)$ & & \\
\hline \multirow[t]{2}{*}{$\mathrm{EtCO}_{2} 43 \mathrm{mmHg}$} & 100 & 11.1 & 20.8 & 100 & $0(0)$ \\
\hline & $(83.9-100)$ & $(5.46-19.49)$ & $(19.61-22.02)$ & & \\
\hline
\end{tabular}

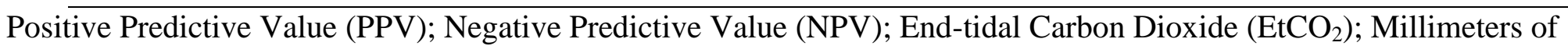
Mercury (mmHg); Confidence Interval (CI) 
Figure 4.1. ROC Curve for End-tidal Carbon Dioxide

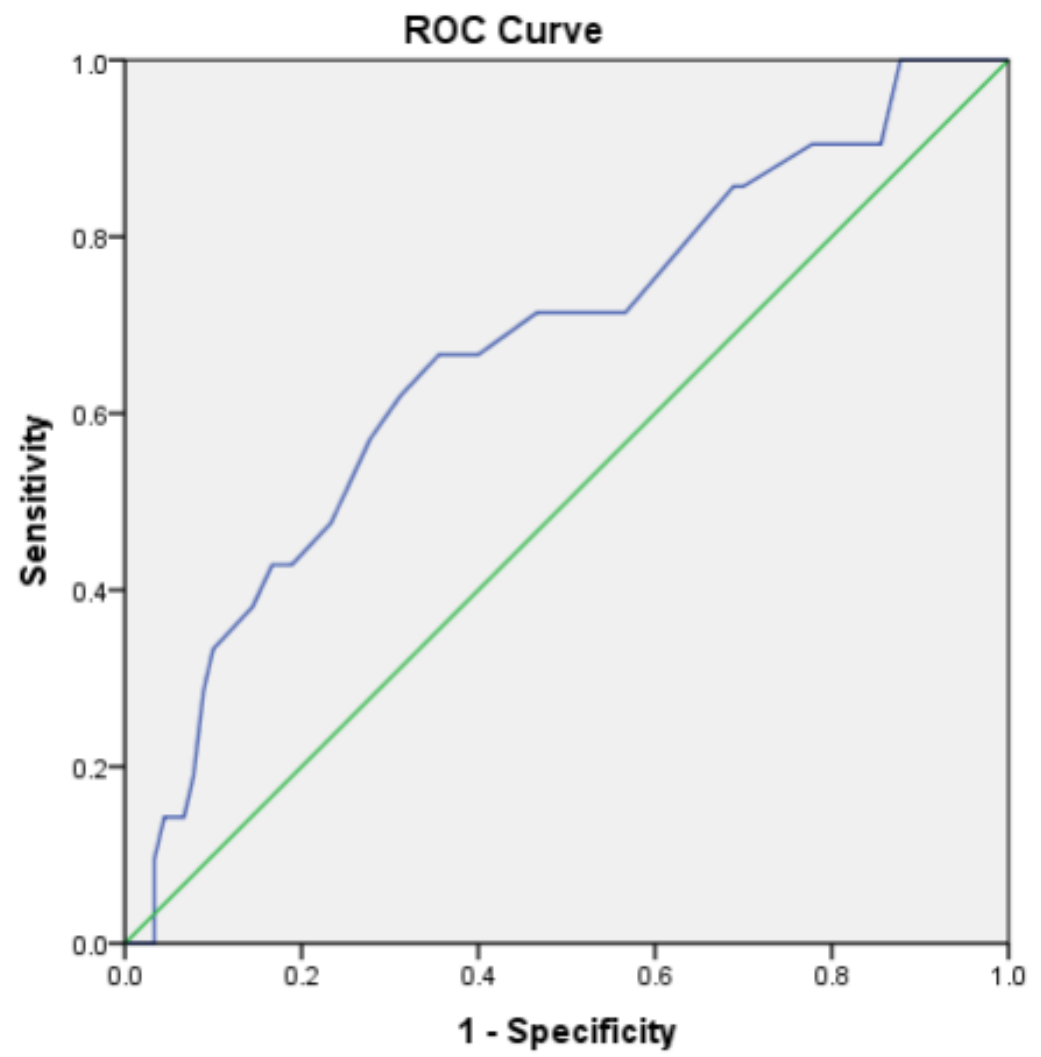

Area under the curve 0.674 Confidence Interval: 0.542, 0.805 


\section{CHAPTER FIVE:}

\section{RESULTS}

Adherence with American Collage of Physician's Best Practice Advice for Diagnosis of Pulmonary Embolism

(Prentice, D. and Wipke-Tevis, D.D.) 


\begin{abstract}
Purpose: As part of a study examining the accuracy and harm avoidance of adding endtidal carbon dioxide measurement to the pulmonary embolism (PE) diagnostic process, we evaluated adherence to the American College of Physicians best practice advice for diagnosis of pulmonary embolism (BPA). Design: The study used a prospective, single center, descriptive design. Methods: A heterogeneous sample of 111 hemodynamically stable hospitalized adult inpatients with a computed tomography pulmonary angiogram (CTPA) ordered was consented. Electronic medical records were reviewed to abstract demographic and clinical variables to determine adherence. The six individual BPA statements and the overall BPA adherence were evaluated by taking the sum of yes answers divided by the sample size. Results: Overall BPA adherence was 0\%. Partial adherence was observed with clinician recorded clinical decisions rules in $3.6 \%(4 / 111)$ of participants. Pulmonary Embolism Rule-Out Criteria (PERC) adherence was 0\%. Ddimer was obtained in $10.2 \%(9 / 88)$ of low/intermediate and $0 \%$ of high probability scorers. All D-dimers were positive; however, age-adjustment was not recorded.

Conclusion: CTPA appears to be the initial PE diagnostic test in up to $90 \%$ of hemodynamically stable, hospitalized adults. Clinical utility of PERC and D-dimer testing in hospitalized adults needs further evaluation. Lack of a standardized PE CDR, laboratory reporting of age-adjusted D-dimer values, and information technology support likely contributed to the poor adherence.
\end{abstract}




\section{Adherence with American Collage of Physician's Best Practice Advice for Diagnosis of Pulmonary Embolism}

Making a prompt accurate diagnosis of pulmonary embolism (PE) often is a clinical challenge. For hemodynamically unstable patients suspected of PE, the diagnostic plan is straightforward; they should proceed directly to computed tomography pulmonary angiography (CTPA), if available (Raja, Greenberg, et al., 2015). For all others, the American College of Physicians (Raja, Greenberg, et al., 2015) provide guidelines and best practices for a standardized approach for PE diagnosis. Specifically, for hemodynamically stable (blood pressure $\geq 90 \mathrm{mmHg}$ ) patients, the PE diagnostic steps include: probability determination using a validated PE clinical decision rule (CDR); Pulmonary Embolism Rule-Out Criteria (PERC) evaluation; D-dimer testing with appropriate age-adjustment; and CTPA imaging (Raja, Greenberg, et al., 2015).

A PE CDR is an objective tool that provides a standardized approach to help clinicians determine the likelihood of a PE diagnosis and whether more definitive diagnostic testing is needed (Raja, Greenberg, et al., 2015). Although PE CDRs have existed for nearly 20 years and usage is recommended in current PE clinical guidelines (Konstantinides et al., 2014; Raja, Greenberg, et al., 2015), PE CDR adherence has not been well-documented in the literature. A recent meta-analysis by Bass and colleagues (2017) found the Wells4 score was more sensitive but less specific when utilized in hospitalized patients compared to ED patients. Thus, the authors concluded that although the Wells4 score is safe to use in hospitalized patients, the Wells4 score may eliminate a 
smaller percentage of hospitalized patients from additional testing (Bass et al., 2017). Similarly, the PERC is a set of clinical criteria recommended for use in patients who fall in the low PE probability based on a validated PE CDR. If all of the PERC criteria are met (i.e. negative PERC) (Age $<50$ years, heart rate $<100 \mathrm{bpm}, \mathrm{SaO}_{2}>94$ on room air, no unilateral leg swelling, no hemoptysis, no surgery/trauma $\leq 4$ weeks, no prior PE of deep vein thrombosis (DVT), no estrogen use), PE is unlikely and no further testing is needed (Kline, Mitchell, Kabrhel, Richman, \& Courtney, 2004; Singh, Mommer, Erwin, Mascarenhas, \& Parsaik, 2013). While the PERC is a useful tool and has been validated in ED patient population, it has not been evaluated for use in the hospitalized patient (J. Kline, personal communication July 10, 2018).

Further diagnostic assessment using a D-dimer test is recommended in patients with a low or intermediate PE probability based on the PE CDR or who were not "ruled out" by the PERC (Kline \& Kabrhel, 2015; Konstantinides et al., 2014; Raja, Greenberg, et al., 2015). D-dimer, a fibrin-derived fragment released into the circulation when fibrin is broken down, is elevated in conditions where any recent blood clotting has occurred. The combination of a low PE probability score from a CDR with a negative D-dimer has a negative predictive value (NPV) of 95\% and can safely exclude PE (Kline et al., 2012; Konstantinides et al., 2014; Long \& Koyfman, 2016; Raja, Greenberg, et al., 2015). Patients testing positive for D-dimer need to undergo a CTPA for definitive diagnosis (Raja, Greenberg, et al., 2015). 
The CTPA, a multi-detector scan, has replaced the catheter pulmonary angiogram and ventilation to perfusion (V/Q) scan as the accepted gold standard test for PE diagnosis (Konstantinides et al., 2014; Long \& Koyfman, 2016; Raja, Greenberg, et al., 2015). CTPA is fast, reliable and easily accessible in most clinical settings. The CTPA obtains high resolution images of the pulmonary vasculature while a bolus of intravenous (IV) contrast material travels through the pulmonary vasculature allowing for detection of a filling defect or lack of blood flow. Improved technology has made the CTPA both sensitive (95\%) and specific (96\%) for PE diagnosis (Meinel et al., 2015; Parikh et al., 2015).

In the last five years, researchers have examined clinician adherence to PE diagnostic guidelines originating from a variety of organizations including 2006 PIOPED II, 2008 European Society of Cardiology, 2011 American College of Emergency Physicians, 2014 European Society of Cardiology, as well as an institutional algorithm (Alhassan et al., 2016; Chandra et al., 2013; Perelas et al., 2015a; Perera, Aggarwal, Scott, \& Cocks, 2017; Yan et al., 2017; Yin et al., 2012). Collectively, these studies reveal that CTPA is being done on patients with low clinical probability based on a PE CDR, in patients without D-dimer testing and/or in patients with a negative D-dimer (Alhassan et al., 2016; Perera et al., 2017; Yan et al., 2017). Clinicians may override guidelines and order a CTPA in hopes of finding alternative causes for the patient's signs and symptoms; however, current research does not suggest this is an effective strategy (Chandra et al., 2013; van Es et al., 2013). To our knowledge, no prior research has 
evaluated adherence to the recent 2015 American College of Physicians guidelines for the diagnosis of acute PE in hospitalized patients (Raja, Greenberg, et al., 2015). Therefore, as part of a study examining the accuracy and harm avoidance of adding end tidal carbon dioxide $\left(\mathrm{EtCO}_{2}\right)$ measurement to the PE diagnostic process (Prentice et al., in preparation), we evaluated clinician adherence to the 2015 American College of Physicians Evaluation of Patients with Suspected Acute Pulmonary Embolism Best Practice Advice (BPA) (Raja, Greenberg, et al., 2015). We had two research questions: 1) What is the overall rate of adherence to the 2015 American College of Physicians guidelines for the diagnosis of acute PE?, and 2) What is the rate of adherence to the 2015 American College of Physicians six individual best practice advice statements for the diagnosis of acute PE?

\section{Methods}

\section{Study Procedures}

This single center, prospective study was performed at a large, Midwestern academic medical center in a major metropolitan area. The study was approved for waiver of documentation of consent by the University of Missouri Institutional Review Board (IRB; Appendix A) as well as the Research Review Committee at the study site (Appendix B). All English speaking, hospitalized patients 18 years of age or older who were hemodynamically stable (systolic blood pressure $\geq 90 \mathrm{mmHg}$ ) and had a CTPA ordered to rule out PE during the study period were eligible for enrollment. Prisoners and patients with the following treatments or conditions were excluded: non-mechanically 
ventilated tracheostomy, Optiflow ${ }^{\circledR}$ nasal cannula, and pregnancy. Eligible patients were referred to the research team by the radiology department. Participants or their legally authorized representative were approached by the principal investigator and informed consent was obtained. Relevant clinical and demographic variables necessary to answer to study questions were extracted from the electronic medical record (EMR).

PE Clinical Decision Rule Determination. For participants with a PE CDR documented in the EMR, the PI recorded the CDR score and/or interpretation. For participants without a documented PE CDR, the PI calculated a traditional Wells score based on EMR data. Information needed to calculate the Wells score included: alternative diagnosis less likely, heart rate $>100$, hemoptysis, history of venous thromboembolism (VTE), immobilization ( $>3$ days), recent surgery/trauma ( $\leq 4$ weeks), suspected DVT, and treated malignancy ( $<6$ months) or palliative care (Lucassen et al., 2011; Wells et al., 2000). Given that all participants had to have a CTPA ordered to rule out PE to be eligible for study inclusion, the PI counted the "PE considered the likely diagnosis" for the Wells calculations unless specifically stated otherwise in the EMR.

Pulmonary Embolism Rule-Out Criteria (PERC) Evaluation. Since the PERC was not utilized at the study site, the PI also evaluated each participant using the PERC criteria based upon EMR data. Data extracted to evaluate the PERC criteria included: age, heart rate, hemoptysis, hormone use, prior DVT or PE, pulse oximetry, recent surgery or trauma ( $\leq 4$ weeks), and unilateral leg swelling (Kline \& Kabrhel, 2015; Kline, 
Mitchell, et al., 2004; Raja, Greenberg, et al., 2015). Physiologic data (i.e. heart rate; pulse oximetry) was recorded at the time of CTPA order.

D-dimer. For participants whose clinician ordered a D-dimer laboratory test, a blood sample was obtained (blue-top (3.2\% sodium citrate) tube) and a quantitative Ddimer was performed using an agglutination method on an IL-Tops coagulation instrument (Instrumentation Laboratory, Bedford, MA) according to the hospital's standard operating procedures. If ordered and available in the EMR, the PI abstracted the D-dimer test results. For participants $\geq 50$ years old, the PI performed an age-adjusted Ddimer calculation (age x 10ng/ml) (Woller et al., 2014). At the study site, the normal laboratory range for D-dimer was $<500$ nanograms $(\mathrm{ng}) / \mathrm{ml}$. The PI did not draw or order D-dimer testing for any study participants.

CTPA. All study participants had a CTPA completed with a multi slice spiral scan according to the standard operating procedures of the study site. The $11 \mathrm{CT}$ scanners used for the diagnosis of PE were made by Siemens Medical (40 Liberty Boulevard, Malvern, PA 19355). The PI extracted the CTPA results from the final CTPA report in the EMR.

\section{Data Management and Analysis}

De-identified data were analyzed with the assistance of the study statistician using IBM SPSS v24 (IBM Corporation, Armonk, New York) statistical software. General descriptive statistics were used to summarize the data. Continuous variables are reported 
as a mean \pm standard deviation and analyzed by an independent t-test; categorical variables are reported as percentages and analyzed by Chi-squared test or Fisher's exact test in the case of small cell sizes. To determine the overall rate of adherence to the 2015 American College of Physicians guidelines for the diagnosis of acute PE, the overall percent of adherence was calculated as the number of yes answers divided by the total number of subjects. Each standard was evaluated separately to determine overall percentage of adherence. To determine the rate of adherence to the six individual best practice advice statements for the diagnosis of acute PE, the statements were individually examined and assigned a yes/no. The rate of adherence was calculated taking the sum of yes answers divided by the total number of participants for each individual statement. Each individual statement was evaluated to see if there was one statement that was causing the overall adherence to be lower.

\section{Results}

A convenience sample of 114 participants was consented; however, 2 did not have a CTPA scans completed and 1 had an indeterminate CTPA scan result. Thus, the total number of participants included for analysis was 111 (41 male; 70 female). The mean $( \pm \mathrm{SD})$ age of the sample was $59( \pm 15.9)$ years old. All hospital services were represented in the sample with the most frequent being medical $43.2 \%(48 / 111)$, surgical $33.3 \%$ (37/111) and oncology 20.7\% (23/111). The majority of participants were located on the general medical-surgical hospital divisions $80 \%(89 / 111)$ rather than the intensive care units (ICU) 20\% (22/111); however, the proportion of participants located on the 
general medical-surgical units and intensive care units were not different in PE positive (general division 71.4\% (15/21); ICU 28.6\% (6/21)) and PE negative participants (general division 81.1\% (73/91); ICU 18.9\% (17/90); $P=.324)$.

Best Practice Advice Statement 1. Clinicians should use validated clinical prediction rules to estimate pretest probability in patients in whom acute PE is being considered (Raja, Greenberg, et al., 2015, p. 701).

Only 4 of 111 participants had a PE CDR documented in the EMR. All documented PE CDRs were the traditional Wells score. Two participants were categorized as low PE probability with scores of 1.5; two participants were categorized intermediate PE probability with scores of 4 and 4.5. Thus, adherence to Best Practice Advice Statement 1 was $3.6 \%$. Of note, none of these participants with a clinician documented low/intermediate PE CDR score were PE positive by CTPA. PI calculated traditional Wells scores for all study participants are displayed in Table 5.1. The majority of study participants $(77.5 \% ; 86 / 111)$ fell into the intermediate probability category.

\section{Best Practice Advice Statement 2. Clinicians should not obtain D-dimer measurements or imaging studies in patients with a low pre-test probability of PE and who meet all Pulmonary Embolism Rule-Out Criteria (PERC) (Raja, Greenberg,} et al., 2015, p. 701).

Although two participants had clinician documented traditional Wells scores categorizing them as low PE probability, none had a PERC evaluation documented in the 
EMR. Additionally, all participants had a CTPA. Thus, adherence to Best Practice Advice Statement 2 was $0 \%$.

PI calculated PERC evaluations are summarized in Figure 5.1. None of the study participants met the PERC exclusion criteria - suggesting that none of the study participants should be ruled out for PE at this point in the diagnostic algorithm. The most common positive PERC criteria, for both PE positive and negative participants, were age $\geq 50$ years old, oxygen saturation $<94 \%$ on room air, heart rate $>100$ beats per minute and recent surgery/trauma $\leq 4$ weeks. Unilateral leg swelling was the only positive PERC criteria that was more common in PE positive than PE negative participants (Figure 5.1; $P=.021)$.

Best Practice Advice Statement 3. Clinicians should obtain a high sensitivity Ddimer measurement as the initial diagnostic test in patients who have an intermediate pretest probability of $\mathrm{PE}$ or in patients with low pretest probability of PE who do not meet all Pulmonary Embolism Rule-Out Criteria. Clinicians should not use imaging studies as the initial test in patients who have a low or intermediate pretest probability of PE (Raja, Greenberg, et al., 2015, p.701).

Based solely on the clinician documented intermediate PE probability, two study participants met the criteria to have a high sensitivity D-dimer test. Only one of these two participants had a D-dimer obtained while the other participant had CTPA as their initial diagnostic test. Thus, adherence to the first and third criteria of Best Practice Advice Statement 3 was achieved in only 1 participant for a raw adherence of 50\%. However, 
when the PI calculated the traditional Wells score, 86 study participants fell into the intermediate PE probability; thus, actual adherence fell to $1.2 \%$ (1/86). Additionally, one of the low PE probability participants had a D-dimer tested without documentation of the PERC evaluation; demonstrating nonadherence to the second criteria of Best Practice Advice Statement 3 in this participant.

Only nine participants had a D-dimer ordered by their provider. Based on the PI calculated Wells score plus the PI evaluated PERC criteria, 79.2\% (88/111) of the low/intermediate PE probability participants met the criteria to have a D-dimer test. Adherence to Best Practice Advice 3 for obtaining D-dimer was 10.2\% (9/88). All nine study participants (100\%) with a D-dimer had an elevated or positive D-dimer. Contrary to the Best Practice Advice 3, the vast majority of study participants $(89.7 \% ; 79 / 88)$ had CTPA as their initial diagnostic test.

We evaluated the clinical indications that could cause D-dimer to be positive, regardless of a PE diagnosis (Table 5.2). All participants (100\%) had at least two clinical indications with some participants having as many as 12 clinical indications to have an elevated D-dimer. The top four clinical indications for an elevated D-dimer were: surgery, sepsis/infection, cancer and deep venous thrombosis (DVT).

Best Practice Advice Statement 4. Clinicians should use age-adjusted D-dimer thresholds (age $\times 10 \mathrm{ng} / \mathrm{mL}$ ) rather than a generic $500 \mathrm{ng} / \mathrm{mL}$ in patients older than 50 years to determine whether imaging is warranted (Raja, Greenberg, et al., 2015, p.701). 
Five $(55.5 \%)$ of the nine study participants that had D-dimer tested were $\geq 50$ years old and met the criteria for use of the age-adjusted D-dimer value. Age-adjustment of D-dimer values was not part of the standard operating procedures in the laboratory at the study site. Moreover, clinicians did not document age-adjustment of D-dimer values for any study participant. Therefore, adherence to Best Practice Advice 4 was 0\%.

The PI calculated age-adjusted D-dimer values for the five participants who were $\geq 50$ years old. Based on these calculations, one study participant had a normal ageadjusted D-dimer. Seventy-seven percent (68/88) of the PI calculated low/intermediate PE probability participants were $\geq 50$ years old. Thus, had Best Practice Advice 3 and 4 been followed, $77 \%$ of the study participants would have needed a D-dimer test and would have met the criteria for age-adjustment of their D-dimer value.

\section{Best Practice Advice Statement 5. Clinicians should not obtain any imaging studies in patients with a D-dimer level below the age- adjusted cutoff (Raja, Greenberg, et} al., 2015, p.701).

As mentioned above, none of the participants with a D-dimer test had documentation of age-adjustment. Based on PI calculation of the D-dimer ageadjustment in those $\geq 50$ years old, one participant had a normal age-adjusted D-dimer value. The participant with a normal age-adjusted $\mathrm{D}$-dimer value did indeed receive a CTPA and the CTPA was negative for PE. Accordingly, adherence to Best Practice Advice 5 was $0 \%$. 
Best Practice Advice Statement 6. Clinicians should obtain imaging with CT pulmonary angiography (CTPA) in patients with high pretest probability of PE. Clinicians should reserve ventilation-perfusion scans for patients who have a contraindication to CTPA or if CTPA is not available. Clinicians should not obtain a D-dimer measurement in patients with a high pretest probability of PE (Raja, Greenberg, et al., 2015, p.701).

Adherence to Best Practice Advice 6 was $0 \%$ since none of the participants in the PI calculated high PE probability category had a clinician documented CDR in the EMR. PI calculated traditional Wells scores classified $20.7 \%(23 / 111)$ of study participants in the high PE probability group. None of the study participants in the high PE probability group had a D-dimer drawn and all had CTPA as their first test; however, none had a CDR documented. Given that all study participants had to have a CTPA ordered to rule out PE to be eligible for study inclusion, we did not evaluate adherence to the second criterion of Best Practice Advice Statement 6.

\section{Discussion}

This study found adherence to the 2015 American College of Physicians Evaluation of Patients with Suspected Acute Pulmonary Embolism Best Practice Advice, as documented in the EMR, was low in hemodynamically stable, hospitalized patients being evaluated for PE diagnosis using CTPA. In particular, $<5 \%$ had a validated PE CDR and none had a Pulmonary Embolism Rule-Out Criteria evaluation documented in their EMR. Similarly, <10\% of patients meeting criteria had a D-dimer ordered and only 
2 of those had a prior CDR. Moreover, none of the patients $\geq 50$ years old with a Ddimer ordered had documentation of age-adjustment with the D-dimer results. Thus, these data suggest that CTPA continues to be utilized as the initial test in the majority of hemodynamically stable, hospitalized patients who are being evaluated for a PE diagnosis.

\section{Use of a Validated Clinical Decision Rule}

Use of a validated PE CDR as the first step to the PE diagnostic process is currently recommended not only by the American College of Physicians (Raja, Greenberg, et al., 2015), but also by the European Society of Cardiology (Konstantinides et al., 2014). In lieu of a PE CDR, the alternative would be utilization of gestalt, or best guess, based on the provider's prior clinical experiences. Indeed, some research has demonstrated similar accuracy between a PE CDR and an experienced provider's gestalt (Kline \& Kabrhel, 2015; Lucassen et al., 2011). However, in a teaching hospital, such as the setting in which this study was conducted; a PE CDR may be particularly useful as it provides a logical, stepwise approach for providers with less clinical experience.

Our findings in a heterogeneous sample of hospitalized participants are consistent with prior research demonstrating PE CDRs are underutilized in clinical practice. Specifically, two recent studies examined the use of PE CDRs in female emergency department patients (ED) (Bickley et al., 2015) and a heterogeneous sample of ED and hospitalized patients (Perera et al., 2017) being evaluated for a PE. Both studies found $<10 \%$ of the patients had a PE CDR score documented in the EMR. Reported obstacles 
to PE CDR use include clinician resistance, lack of CDR incorporation into the EMR, fear of legal reprisal if a PE is overlooked, and lack of familiarity with CTPA risks (Sherk \& Stojanovska, 2017). Future research is needed to identify strategies to improve CDR usage.

For hospitalized patients, the study site does not require documentation of a PE CDR as a pre-requisite to ordering a CTPA. This is particularly interesting given that ED clinicians are required to utilize a DVT CDR (Wells et al., 1997) prior to ordering a compression ultrasound (CUS). It is unclear from this study why a DVT CDR is required to order a non-invasive CUS with minimal risks but a PE CDR is not required to order an invasive CTPA which carries allergic, renal, and radiation risks. As a result, in this setting, the clinician evaluating a patient suspected of $\mathrm{PE}$ is on their own to remember to select a validated PE CDR, calculate the PE probability, and document and interpret the PE CDR score without support of an integrated clinical decision support system within the EMR.

Use of the Pulmonary Embolism Rule-Out Criteria (PERC). Although use of the PERC evaluation is addressed in Best Practice Advice Statements 2 and 3 and the 2015 BPA is described as being applicable to both inpatients and outpatients (Raja, Greenberg, et al., 2015, p 702) to our knowledge, the clinical utility of the PERC evaluation has not been previously examined in a heterogeneous population of hospitalized patients being evaluated for PE. Based on the PI's assessment, none of the study participants would have met the criteria to be ruled out for PE based on the PERC 
evaluation. Moreover, all study participants had multiple positive PERC criteria. Thus, even if the PERC criteria had been utilized according to the BPA, none of the study participants would have been excluded from PE diagnosis and all participants would have needed further diagnostic testing. These data suggest that while the PERC is a valuable tool to rule-out PE in patients with low PE probability in the ED setting (Kline, Webb, Jones, \& Hernandez-Nino, 2004; Singh et al., 2013), the PERC may not be a beneficial diagnostic step when evaluating acutely and/or chronically ill inpatients with risk factors and/or symptoms suggestive of $\mathrm{PE}$ such as age $\geq 50$ years, recent surgery or trauma, $\mathrm{SaO}_{2}$ $<95 \%$, and tachycardia. Additional research is needed with a larger sample of heterogeneous inpatients being evaluated for PE diagnosis to determine clinical utility of the PERC in the hospitalized patient population.

\section{High sensitivity D-dimer Testing}

The real value of $\mathrm{D}$-dimer testing comes from using a negative value to exclude the PE diagnosis (Kabrhel et al., 2010; Kline et al., 2012) and, correspondingly, eliminate the need for further diagnostic testing. Current research and best practices both support the view that a negative, high sensitivity D-dimer in conjunction with a low PE probability score from a validated CDR can rule out PE with a 95\% negative predictive value (Crawford et al., 2016; Douma et al., 2011; Kabrhel et al., 2010; Kline et al., 2012; Lapner \& Kearon, 2013; Riley, Gilbert, Dalton, Pai, \& McPherson, 2016). Unfortunately, there are many positive D-dimer results caused by recent trauma, surgery or invasive procedure, inflammation, pregnancy, renal disease, African American race, advancing 
age or malignancy (Riley et al., 2016). While only a small portion of our study participants had D-dimer testing, we found that all study participants had two or more possible clinical reasons, unrelated to a PE diagnosis, for their D-dimer to be elevated. Indeed, it is possible that the reason D-dimer testing was completed in $<10 \%$ of study participants is that providers recognized that their patient's D-dimer likely would be positive based on their clinical presentation. Given that D-dimer is nonspecific in regards to etiology or location of the fibrin clot (Riley et al., 2016), the clinical utility of a Ddimer testing in hospitalized adults with multiple non-PE related causes for D-dimer elevation needs further study

One method of improving the accuracy of D-dimer testing in older adults is to use an age-adjusted normal. Using age-adjustment for people $\geq 50$ is a validated method for D-dimer assessment (Righini et al., 2014) which raises the normal value and helps to eliminate false positive results (Woller et al., 2014). Of note, one of the PE negative study participants could have had their CTPA test eliminated had their provider utilized an age-adjustment for their D-dimer test result. Relying on clinicians to remember to use the D-dimer age-adjustment calculation increases the opportunity for errors of omission, particularly in a teaching hospital with inexperienced providers. To increase the accuracy of interpreting D-dimer results, age-adjusted normal values could be incorporated into the laboratory normal ranges similar to what is typically done with glomerular filtration rate calculations.

\section{Implications for Research}


Our study has highlighted multiple opportunities for future research. Barriers to adoption of BPA can arise from clinicians, patients or the system (Ryan, 2017). While not examined in our study, the clinician perspective needs to be explored in regards to what support would improve adherence with BPA and what factors may cause the clinician to override the BPA. Additionally, research comparing clinician years of experience (resident, junior attending, senior attending), clinician academic preparation (advanced practice nurse, physician assistant or physician) and employment type (salaried versus fee for service) could shed additional light on predictors of BPA adherence.

Given that all PI calculated PERC evaluations were positive and all D-dimers were positive, albeit only a small number were tested, the utility of both the PERC evaluation and D-dimer needs further research in the hospitalized patient population. Moreover, these data raise the question of whether additional pre-imaging PE diagnostic strategies are needed - especially in the hospitalized patient population with multiple comorbid conditions. For example, alveolar dead space assessment with end-tidal carbon dioxide $\left(\mathrm{EtCO}_{2}\right)$ monitoring is one emerging diagnostic tool (Prentice \& Wipke-Tevis, In Press; Ramme et al., 2016; Riaz \& Jacob, 2014) that may be a useful adjunct to help exclude additional PE negative patients and minimize costs and risks associated with unnecessary radiologic imaging (Manara, D’hoore, \& Thys, 2013; Prentice et al., In Preparation; Ramme et al., 2016; Riaz \& Jacob, 2014).

Implications for Clinical Nurse Specialists 
One of the aspects of the role of the clinical nurse specialist (CNS) is to translate clinical guidelines into their specific practice setting. Additionally, the CNS is often in a position to evaluate guideline adherence and, when necessary, impact change to improve adherence (Bryant-Lukosius et al., 2015; Derby, Hartung, Wolf, Zak, \& Evenson, 2017). In doing so, an inter-professional team should be assembled. The team should include key stakeholders who have the knowledge to define the problem and generate possible solutions and have the ability to impact change. The CNS as team leader will oversee the process to gather and synthesize the literature, determine the gaps in practice, obtain input from frontline staff, determine an action plan and evaluate the outcome. The Information Technology department should be a key partner to help ensure and improve smooth integration of the BPA into the EMR, tracking adherence and providing feedback regarding hospital, unit and individual rates of adherence performance (Raja, Ip, et al., 2015; Ryan, 2017; Yan et al., 2016). Another opportunity to improve BPA adherence is to utilize the patient in shared decision making (Ryan, 2017). The CNS can assist with increasing patient understanding of the BPA by developing and/or implementing patient and family education tools about the BPA.

\section{Limitations}

The limitations of this study need to be acknowledged. The study used a convenience sample of hospitalized adults with a CTPA ordered to rule out PE. Adherence to the best practice advice might be different if we included all adults being evaluated for PE, rather than only including those with a CTPA ordered. We relied on 
clinician documentation to determine adherence to the BPA. Thus, the findings are limited by the accuracy and completeness of the EMR documentation. Since the study site does not have PE CDR and/or PERC documentation incorporated into their CTPA ordering process within the EMR, their actual usage is unknown. Likewise, given that clinicians were not interviewed, we cannot make any assessment of their knowledge, or lack thereof, of the BPA. Future prospective, multi-center studies are needed to validate the findings and increase the generalizability.

\section{Conclusion}

The American College of Physicians (Raja, Greenberg, et al., 2015) has established best practice advice for the diagnosis of PE to be utilized for both inpatients and outpatients. In hemodynamically stable patients suspected of PE, the best practice advice guides the clinician to estimate pre-test probability determination with a validated PE CDR, assess the Pulmonary Embolism Rule-Out Criteria in patients with low PE probability, order D-dimer testing for patients with low PE probability plus positive PERC or patients with intermediate PE probability prior to any radiologic imaging (Raja, Greenberg, et al., 2015). Radiologic imaging with CTPA is reserved for patients with low/intermediate PE probability plus positive D-dimer or patients with high PE probability (Konstantinides et al., 2014; Raja, Greenberg, et al., 2015). This study found very low documentation of adherence to the current American College of Physicians best practice advice for diagnosis of PE in a heterogeneous sample of hospitalized patients. Furthermore, we found a number of system problems that likely contributed to the poor 
adherence such as lack of a standardized PE CDR, lack of laboratory reporting of ageadjusted D-dimer values, and lack of information technology support (i.e. clinical decision support) imbedded within the EMR. Organizational change will be necessary to improve BPA adherence. Involvement of key stakeholders in the design, implementation, and evaluation of the new processes will be necessary to ensure that changes are implemented smoothly and successfully facilitate clinician adherence to the BPA. 


\section{References}

Alhassan, S., Sayf, A. A., Arsene, C., \& Krayem, H. (2016). Suboptimal implementation of diagnostic algorithms and overuse of computed tomography-pulmonary angiography in patients with suspected pulmonary embolism. Annals of Thoracic Medicine, 11(4), 254-260. doi:10.4103/1817-1737.191875

Bass, A., Fields, K., Goto, R., Turissini, G., Dey, S., \& Russell, L. (2017). Clinical decision rules for pulmonary embolism in hospitalized patients: A systematic literature review and meta-analysis. Thrombosis and Haemostasis, 117(11), 2176-2185. doi:10.1160/TH17-06-0395

Bickley, D., Lindsey, J., Saint-Hilaire, R., Hussain, A., Vu, M., MacMahon, H., ... Walter, J. (2015). 678: an analysis of the use of CT pulmonary angiography in young women in an urban emergency department. Critical Care Medicine, 43, 171. doi:10.1097/01.ccm.0000474506.44733.8f

Bryant-Lukosius, D., Carter, N., Reid, K., Donald, F., Martin-Misener, R., Kilpatrick, K., ... DiCenso, A. (2015). The clinical effectiveness and cost-effectiveness of clinical nurse specialist-led hospital to home transitional care: A systematic review: CNS-led transitional care. Journal of Evaluation in Clinical Practice, 21(5), 763-781. doi:10.1111/jep.12401

Chandra, S., Sarkar, P. K., Chandra, D., Ginsberg, N. E., \& Cohen, R. I. (2013). Finding an alternative diagnosis does not justify increased use of CT-pulmonary 
angiography. BMC Pulmonary Medicine, 13(1), 1-8. doi:doi:10.1186/1471-246613-9

Crawford, F., Andras, A., Welch, K., Sheares, K., Keeling, D., \& Chappell, F. M. (2016). D-dimer test for excluding the diagnosis of pulmonary embolism. Cochrane Database of Systematic Reviews. doi:10.1002/14651858.CD010864.pub2

Derby, K. M., Hartung, N. A., Wolf, S. L., Zak, H. L., \& Evenson, L. K. (2017). Clinical Nurse Specialist-Driven Practice Change: Standardizing Vital Sign Monitoring. Clinical Nurse Specialist, 31(6), 343-348. doi:10.1097/NUR.0000000000000330

Douma, R. A., Mos, I. C., Erkens, P. M., Nizet, T. A., Durian, M. F., Hovens, M. M., ... Huisman, M. V. (2011). Performance of 4 clinical decision rules in the diagnostic management of acute pulmonary embolism: A prospective cohort study. Annals of Internal Medicine, 154(11), 709-718. doi:10.7326/0003-4819-154-11201106070-00002

Kabrhel, C., Mark Courtney, D., Camargo, C. A., Plewa, M. C., Nordenholz, K. E., Moore, C. L., ... Kline, J. A. (2010). Factors associated with positive d-dimer results in patients evaluated for pulmonary embolism: Factors associated with positive d-dimers. Academic Emergency Medicine, 17(6), 589-597. doi:10.1111/j.1553-2712.2010.00765.x

Kline, J. A., Hogg, M. M., Courtney, D. M., Miller, C. D., Jones, A. E., \& Smithline, H. A. (2012). D-dimer threshold increase with pretest probability unlikely for 
pulmonary embolism to decrease unnecessary computerized tomographic pulmonary angiography: Elevated D-dimer threshold. Journal of Thrombosis and Haemostasis, 10(4), 572-581. doi:10.1111/j.1538-7836.2012.04647.x

Kline, J. A., \& Kabrhel, C. (2015). Emergency evaluation for pulmonary embolism, part 2: Diagnostic approach. The Journal of Emergency Medicine, 49(1), 104-117. doi:10.1016/j.jemermed.2014.12.041

Kline, J. A., Mitchell, A. M., Kabrhel, C., Richman, P. B., \& Courtney, D. M. (2004). Clinical criteria to prevent unnecessary diagnostic testing in emergency department patients with suspected pulmonary embolism. Journal of Thrombosis and Haemostasis, 2(8), 1247-1255. doi:10.1111/j.1538-7836.2004.00790.x

Kline, J. A., Webb, W. B., Jones, A. E., \& Hernandez-Nino, J. (2004). Impact of a rapid rule-out protocol for pulmonary embolism on the rate of screening, missed cases, and pulmonary vascular imaging in an urban US emergency department. Annals of Emergency Medicine, 44(5), 490-502.

Konstantinides, S. V., Torbicki, A., Agnelli, G., Danchin, N., Fitzmaurice, D., Galiè, N., ... Spyropoulos, A. C. (2014). 2014 ESC Guidelines on the diagnosis and management of acute pulmonary embolism. European Heart Journal, 35(43), 3033-3080. doi:10.1093/eurheartj/ehu283

Lapner, S. T., \& Kearon, C. (2013). Diagnosis and management of pulmonary embolism. BMJ, 346, f757. doi:10.1136/bmj.f757 
Long, B., \& Koyfman, A. (2016). Best clinical practice: current controversies in pulmonary embolism imaging and treatment of subsegmental thromboembolic disease. The Journal of Emergency Medicine. Retrieved from http://www.sciencedirect.com/science/article/pii/S0736467916306801

Lucassen, W., Geersing, G.-J., Erkens, P. M., Reitsma, J. B., Moons, K. G., Büller, H., \& van Weert, H. C. (2011). Clinical decision rules for excluding pulmonary embolism: A meta-analysis. Annals of Internal Medicine, 155(7), 448-460.

Manara, A., D'hoore, W., \& Thys, F. (2013). Capnography as a diagnostic tool for pulmonary embolism: A meta-analysis. Annals of Emergency Medicine, 62(6), 584-591. doi:10.1016/j.annemergmed.2013.04.010

Meinel, F. G., Nance, J. W., Schoepf, U. J., Hoffmann, V. S., Thierfelder, K. M., Costello, P., ... Bamberg, F. (2015). Predictive value of computed tomography in acute pulmonary embolism: Systematic review and meta-analysis. The American Journal of Medicine, 128(7), 747-759.e2. doi:10.1016/j.amjmed.2015.01.023

Parikh, N., Morris, E., Babb, J., Wickstrom, M., McMenamy, J., Sharma, R., ... Kim, D. (2015). MDCT diagnosis of acute pulmonary embolism in the emergent setting. Emergency Radiology, 22(4), 379-384. doi:10.1007/s10140-014-1290-5

Perelas, A., Dimou, A., Saenz, A., Rhee, J. H., Teerapuncharoen, K., Rowden, A., \& Eiger, G. (2015). CT pulmonary angiography utilization in the emergency 
department: Diagnostic yield and adherence to current guidelines. American Journal of Medical Quality, 30(6), 571-577. doi:10.1177/1062860614543302

Perera, M., Aggarwal, L., Scott, I. A., \& Cocks, N. (2017). Underuse of risk assessment and overuse of CTPA in patients with suspected pulmonary thromboembolism: Overuse of CTPA in suspected PTE. Internal Medicine Journal. doi:10.1111/imj.13524

Prentice, D. and Wipke-Tevis, D. D. (2018). Diagnosis of pulmonary embolism: Following the evidence from suspicion to certainty, Journal of Vascular Nursing, In Press

Raja, A. S., Greenberg, J. O., Qaseem, A., Denberg, T. D., Fitterman, N., \& Schuur, J. D. (2015). Evaluation of patients with suspected acute pulmonary embolism: best practice advice from the clinical guidelines committee of the American college of physicians. Annals of Internal Medicine, 163(9), 701. doi:10.7326/M14-1772

Raja, A. S., Ip, I. K., Dunne, R. M., Schuur, J. D., Mills, A. M., \& Khorasani, R. (2015). Effects of performance feedback reports on adherence to evidence-based guidelines in use of CT for evaluation of pulmonary embolism in the emergency department: a randomized trial. American Journal of Roentgenology, 205(5), 936940. doi:10.2214/AJR.15.14677

Ramme, A. J., Iturrate, E., Dweck, E., Steiger, D. J., Hutzler, L. H., Fang, Y., ... Sigmund, A. E. (2016). End tidal carbon dioxide as a screening tool for computed 
tomography angiogram in postoperative orthopaedic patients suspected of pulmonary embolism. The Journal of Arthroplasty, 31(10), 2348-2352. doi:10.1016/j.arth.2016.03.033

Riaz, I., \& Jacob, B. (2014). Pulmonary embolism in Bradford, UK: role of end-tidal $\mathrm{CO} 2$ as a screening tool. Clinical Medicine, 14(2), 128-133. doi:10.7861/clinmedicine.14-2-128

Righini, M., Van Es, J., Den Exter, P. L., Roy, P.-M., Verschuren, F., Ghuysen, A., ... Le Gal, G. (2014). Age-adjusted d-dimer cutoff levels to rule out pulmonary embolism: the adjust-pe study. JAMA, 311(11), 1117. doi:10.1001/jama.2014.2135

Riley, R. S., Gilbert, A. R., Dalton, J. B., Pai, S., \& McPherson, R. A. (2016). Widely used types and clinical applications of d-dimer assay. Laboratory Medicine, 47(2), 90-102. doi:10.1093/labmed//mw001

Ryan, M. A. (2017). Adherence to Clinical Practice Guidelines. Otolaryngology-Head and Neck Surgery, 157(4), 548-550. doi:10.1177/0194599817718822

Sherk, W. M., \& Stojanovska, J. (2017). Role of clinical decision tools in the diagnosis of pulmonary embolism. American Journal of Roentgenology, 208(3), W60-W70. doi:10.2214/AJR.16.17206

Singh, B., Mommer, S. K., Erwin, P. J., Mascarenhas, S. S., \& Parsaik, A. K. (2013). Pulmonary embolism rule-out criteria (PERC) in pulmonary embolism- 
revisited: A systematic review and meta-analysis. Emergency Medicine Journal, 30(9), 701-706. doi:10.1136/emermed-2012-201730

van Es, J., Douma, R. A., Schreuder, S. M., Middeldorp, S., Kamphuisen, P. W., Gerdes, V. E., \& Beenen, L. F. (2013). Clinical impact of findings supporting an alternative diagnosis on CT pulmonary angiography in patients with suspected pulmonary embolism. CHEST Journal, 144(6), 1893-1899.

Wells, P., Anderson, D., Rodger, M., Ginsberg, J., Kearon, C., Gent, M., ... Hirsh, J. (2000). Derivation of a simple clinical model to categorize patients' probability of pulmonary embolism: Increasing the models utility with the simplified d-dimer. Thrombosis and Haemostasis, 83(03), 416-420. doi:10.1055/s-0037-1613830

Woller, S. C., Stevens, S. M., Adams, D. M., Evans, R. S., Lloyd, J. F., Snow, G. L., ... Elliott, C. G. (2014). Assessment of the safety and efficiency of using an ageadjusted d-dimer threshold to exclude suspected pulmonary embolism. Chest, 146(6), 1444-1451. doi:10.1378/chest.13-2386

Yan, Z., Ip, I. K., Raja, A. S., Gupta, A., Kosowsky, J. M., \& Khorasani, R. (2016). Yield of CT pulmonary angiography in the emergency department when providers override evidence-based clinical decision support. Radiology, 282(3), 717-725.

Yin, F., Wilson, T., Della Fave, A., Larsen, M., Yoon, J., Nugusie, B., ... Chow, R. D. (2012). Inappropriate use of D-dimer assay and pulmonary CT angiography in the 
evaluation of suspected acute pulmonary embolism. American Journal of Medical Quality, 27(1), 74-79. doi:10.1177/1062860611407907 
Table 5.1. PI Calculated Traditional Wells Score from Electronic Medical Record.

\begin{tabular}{lccc}
$\begin{array}{l}\text { Traditional Wells } \\
\text { Clinical Decision Rule }\end{array}$ & $\begin{array}{c}\text { Total Sample } \\
(\mathbf{n = 1 1 1}) \\
\mathbf{n}(\boldsymbol{\%})\end{array}$ & $\begin{array}{c}\text { PE Positive } \\
(\mathbf{n = 2 1 )} \\
\mathbf{n}(\boldsymbol{\%})\end{array}$ & $\begin{array}{c}\text { PE Negative } \\
(\mathbf{n = 9 0 )} \\
\mathbf{n}(\boldsymbol{\%})\end{array}$ \\
\hline & & & \\
< 2 Low probability & $2(1.8)$ & 0 & $2(2.2)$ \\
2-6 Intermediate Probability & $86(77.5)$ & $12(57.1)$ & $74(82.2)$ \\
$>$ 6 High Probability & $23(20.7)$ & $9(42.9)^{*}$ & $14(15.6)$
\end{tabular}

Pulmonary embolism (PE); Principal Investigator (PI); *A higher proportion of the high PE probability participants were in the PE positive group; $P=0.019$ 
Table 5.2 Clinical Indications for D-dimer Results to be Positive.

\begin{tabular}{lc} 
Clinical Indicators & $\begin{array}{c}\text { Total Sample } \\
(\mathbf{n}=\mathbf{1 1 1}) \\
\mathbf{n}(\boldsymbol{\%})\end{array}$ \\
Surgery $\leq \mathbf{4}$ weeks & $47(42.3)$ \\
Current Sepsis/Infection & $44(39.6)$ \\
Active Cancer & $42(37.8)$ \\
Current DVT & $34(30.6)$ \\
African American & $31(27.9)$ \\
Current Smoker & $21(18.9)$ \\
Immobility $\geq \mathbf{4}$ days & $16(14.4)$ \\
Invasive procedure $\leq \mathbf{4}$ weeks & $10(9.0)$ \\
Hemodialysis & $9(8.1)$ \\
CAD & $9(8.1)$ \\
Sickle cell & $3(2.7)$ \\
Rheumatoid Arthritis & $3(2.7)$ \\
Post-partum $\leq \mathbf{4}$ weeks & $3(2.7)$ \\
Seizure & $1(0.9)$ \\
Interpretation: 100\% had clinical indicators for elevation of D- \\
dimer. Mean number of indicators was 5 (range 2-12). \\
\hline Deep Vein Thrombosis (DVT); Coronary Artery Disease (CAD)
\end{tabular}


Figure 5.1 Frequency of Positive Pulmonary Embolism Rule Out (PERC) Criteria by Group.

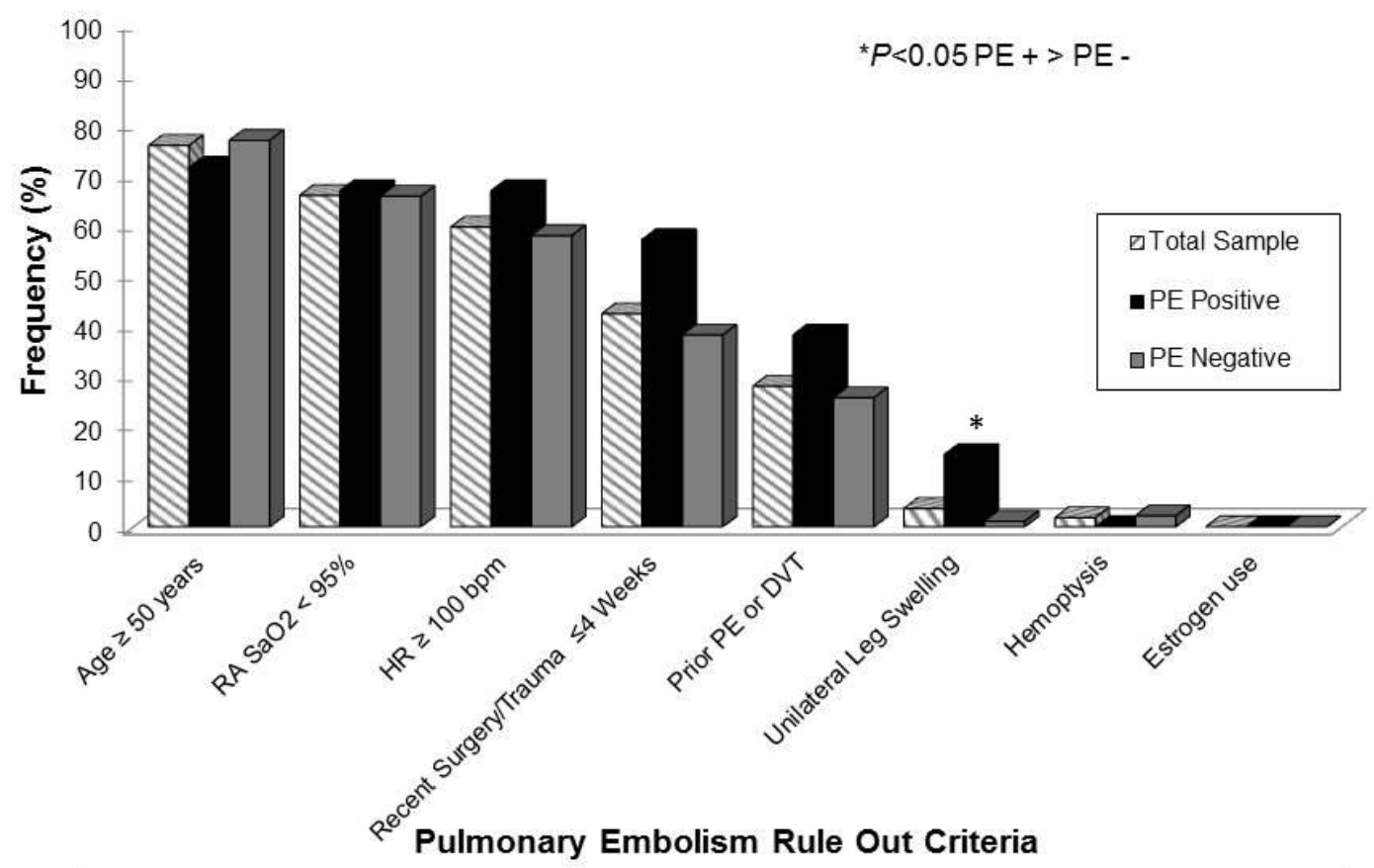

Room Air (RA); Heart Rate (HR); Pulmonary Embolism (PE); Deep Vein Thrombosis (DVT); Pulmonary Embolism Positive(PE+); Pulmonary Embolism Negative(PE-) 


\section{CHAPTER SIX:}

\section{CONCLUSION}

\section{Overview of Dissertation Preparation}

My background as a pulmonary critical care clinical nurse specialist (CNS) has instilled a strong passion for quality care that incorporates, whenever possible, research evidence and best practices. The constant drive for doing the best for staff and patients often led me to find the gaps where evidence was lacking. Over the years, I was fortunate to have mentors, such as Drs. Thom Ahrens and Pat Potter, with whom to work alongside on clinical research to help address those knowledge gaps. Over time, I recognized that in order to be able to undertake larger research projects and to obtain external research funding, I needed a terminal research focused degree. Choosing to pursue my $\mathrm{PhD}$ at the University Of Missouri Sinclair School Of Nursing was based on the school's history of quality education, affordable tuition, a location that was close enough for campus visits and the ability to have a part-time plan of study with distance-mediated coursework.

My phenomenon of interest for doctoral work was the use of end-tidal carbon dioxide $\left(\mathrm{EtCO}_{2}\right)$ in the diagnostic process for pulmonary embolism (PE). While the required courses in proposal writing, research methods, statistics, and theory expanded the depth and breadth of my research knowledge, there were some particular courses that stand out. One of those was Technology Evaluation in Healthcare Research, a substantive science course that was taught by Drs. Alexander and Wakefield. Learning how to undertake a heuristic evaluation is a skill that I could immediately apply in my 
role as a CNS. In class, we applied the heuristic evaluation to the state nursing associations' websites. As a class, we turned this exercise into a scholarly manuscript entitled A usability evaluation exploring the design of American Nurses Association web sites that was published in 2014 in the Computers, Informatics, Nursing journal. One of the most valuable lessons, beyond the course content, was gaining an understanding about the persistence that is required to publish the manuscript. While it was frustrating to get rejections, it was an excellent opportunity to see the effort that goes with publication in a scholarly journal which was a valuable learning experience for my doctoral education, in general, and my doctoral dissertation, in particular.

The second outstanding class was the Meta-analysis course taught by Dr. Lorraine Phillips which included valuable guest lectures by Drs. Conn and Ruppar. Meta-Analysis was a challenging course on several fronts, but I would not miss the opportunity to take this elective class from one of the top meta-analysis teams in nursing. One of the challenges I faced came as I was preparing to undertake the first meta-analysis on the use of capnography (end-tidal $\mathrm{CO}_{2}$ ) in the diagnosis of PE. As I was gathering the literature, a brand new meta-analysis was published on the very topic I was researching! Naturally, I was devastated and feared that I would have to completely change my topic. After carefully reviewing the newly published meta-analysis, I determined that it examined capnography as a whole and did not differentiate between the various $\mathrm{EtCO}_{2}$ measurement methods (Manara, D’hoore, \& Thys, 2013). As a result, I was expertly guided to reframe the focus of my meta-analysis to evaluate the three different types of 
methods that used $\mathrm{EtCO}_{2}$ for the PE diagnostic process. The results of my meta-analysis provided the vision to use the simplest method of $\mathrm{EtCO}_{2}$ which was the absolute $\mathrm{EtCO}_{2}$ value for my dissertation research. The alveolar dead space fraction or $\mathrm{EtCO}_{2}$ gradient options required a mathematical calculation and an invasive and, otherwise unnecessary, arterial blood gas. Moreover, the strength of the evidence supporting use of absolute $\mathrm{EtCO}_{2}$ value was similar to the other methods and it was the least expensive and least invasive method.

The Meta-Analysis course not only assisted me in deciding on the $\mathrm{EtCO}_{2}$ measurement method for my future dissertation research, but also provided me with scholarly dissemination opportunities. For example, after the course was completed, I worked with my advisor to prepare research abstracts, develop and present poster and podium presentations for local, regional and national research conferences (MU Health Science Research Day, Barnes-Jewish Hospital Multidisciplinary Research Day, Midwest Nursing Research Society Annual Research Conference, Society of Critical Care Medicine Annual Congress) and publish a research abstract (Prentice, 2014). In particular, the opportunity to present my $\mathrm{EtCO}_{2}$ meta-analysis at the Society of Critical Care Medicine (SCCM) conference, helped to establish my credibility in this area of research which, subsequently, contributed to my ability to successfully obtain a $\$ 500$ research grant (SCCM Shark Bait Competition) to support my dissertation research. Moreover, while gathering information for the meta-analysis, I was able to speak with Dr. Ann Hemnes, who completed the largest study to date on the use of absolute $\mathrm{EtCO}_{2}$ 
value in the diagnosis of PE (Hemnes et al., 2010), to clarify questions about her study. Establishing this research connection already has been valuable, and we recently have discussed the possibility of future $\mathrm{EtCO}_{2}$ research collaborations.

The research practicums provided very practical research experiences which further prepared me for my dissertation research. I did extensive data base work with Dr. Wipke-Tevis for her physiologic research. Learning how Dr. Wipke-Tevis coded and set up her data was valuable for when it was time for me to do the same for my dissertation research. Data management is an important aspect of dissertation research. This research practicum helped me learn the process of data management including; careful double entry of data, evaluating data for mistakes and clarifying discrepancies. Learning to ensure that date entry is accurate will ensure the data analysis is accurate.

Another research practicum allowed me to spend time with one of the most experienced researchers in the field of pulmonary embolism, Jeffrey Kline, MD, at the University of Indiana in Indianapolis. Dr. Kline has spent years evaluating many forms of $\mathrm{EtCO}_{2}$ in the diagnosis of PE. Additionally, he developed and tested the Pulmonary Embolism Rule-Out Criteria (PERC) criteria. He was willing to share his "lessons learned" and how over the years his research changed from the complex $\mathrm{EtCO}_{2}$ assessment methods to the more simple absolute $\mathrm{EtCO}_{2}$ value. I spent time helping with his current research using an absolute $\mathrm{EtCO}_{2}$ value. I was given access to his data base and I helped to clean, organize and to begin basic analysis. This research practicum really solidified the idea of the simple absolute value of $\mathrm{EtCO}_{2}$ as the method to use in 
my research. The best part of this research practicum was it allowed me to build a relationship with a skilled researcher in a common area of interest. I have continued to communicate with Dr. Kline and he has provided valuable insight into the use of the PERC criteria in the 2015 American College of Physician's Best Practice Advice for the Diagnosis of Pulmonary Embolism and as part of my dissertation research.

The last but key link in this chain was the ability to have an advisor that had experience in physiologic research. The guidance that comes from a professor that has experience and understanding is priceless. Dr. Wipke-Tevis has shepherded me through the process with a meticulous laser focus where I always knew the next step and what was expected. Quality leadership was an invaluable part of this life journey. Two specific areas where Dr. Wipke-Tevis's leadership was beneficial were in organizing a virtual laboratory meeting between the group of $\mathrm{PhD}$ students she was advising and with the Problems/Literature review research practicum.

The virtual laboratory meeting consisted of Jane Scharf, Marilyn Shepherd, Colleen Becker, Jennifer O’Connor and Poungkamon “Mew” Krisanabud. We met weekly on Collaborate to provide feedback to each other related to abstract writing, conceptual model design, figure and table crafting, interpretation of data analysis, manuscript and poster preparation, and comprehensive examination preparation. This virtual laboratory meeting allowed us to provide peer to peer support along with building research skills. 
During the Problems/literature review research practicum, we grappled with understanding the scope of pulmonary embolism (PE) diagnosis literature, identifying the published research related to the use of $\mathrm{EtCO}_{2}$ for $\mathrm{PE}$ diagnosis, and determining the best audience for a publishable manuscript. The format of the manuscript ended up being a clinical review to help nurses understand the diagnostic method options and best evidence behind the process of diagnosing PE. The manuscript ultimately became an integral part of my dissertation (Chapter 2).

While not directly related to my dissertation topic but important for my professional development of transferable skills, I also obtained a Graduate Certificate in Public Health. One course in particular that stands out was the Zoonosis course, or the study of diseases that can be transmitted from an animal to a human. I was the CNS in a medical intensive care unit (MICU) at that time. The patients in the MICU often have unique viruses and infections that are the result of animal to human interaction. I could bring real world experience to the class and the class brought better pathophysiologic understanding to me. I used that class to educate the MICU staff related to those transmission processes. The immediate application of this class to my real world job occurred with the outbreak of Ebola in West Africa. My unit became the regional Ebola treatment facility. The understanding I gained in the Zoonosis class helped the team at my hospital develop staff education and care processes based on a better knowledge of Ebola. 


\section{Summary \& Synthesis of Findings}

My desire to research the use of $\mathrm{EtCO}_{2}$ came from several places. In my role as the CNS in the MICU, I saw an opportunity to improve care. Working with medical residents, I saw them cast a wide net in order to determine what was causing the patient's illness. This practice led to a lot of unnecessary testing, in particular, unnecessary radiologic imaging. Rule out PE always seemed to be on their differential diagnosis list. In addition, it was our practice to use continuous $\mathrm{EtCO}_{2}$ on all of our mechanically ventilated patients. My observations of the $\mathrm{EtCO}_{2}$ use coupled with my pulmonary background caused me to wonder if we could use the $\mathrm{EtCO}_{2}$ data to help eliminate some of the unnecessary radiologic scans. The pulmonary attending would often re-direct the residents away from the radiology testing for PE. It became apparent that the residents needed guidance to help eliminate testing. Not only did the unnecessary radiologic imaging cause potential harm to the patients, it was a burden on the nurses that had to transport the critically ill patient to and from the radiology department. The overuse of radiologic imaging and need for clear PE diagnostic guidelines further bolstered a desire to complete the dissertation research. I saw potential benefit to patients, nurses, and medical personnel if a quick, easy and accurate bedside test could be found in the diagnosis of PE. While the seed for the research started in the ICU, as I delved into the literature, I saw the wider need that spanned both the outpatient evaluation in the Emergency Department (ED), the general hospital divisions in addition to the ICU. 
The first manuscript (Chapter Two), Diagnosis of Pulmonary Embolism: Following the Evidence from Suspicion to Certainty, provided a state of the art review of the diagnostic process for PE and highlighted the evidence underpinning the current 2015 American College of Physicians Best Practice Advice for the Diagnosis of Pulmonary Embolism (Raja et al., 2015). Written with the bedside clinician in mind, a brief overview of the pathophysiology of PE was provided along with a cartoon depicting the pathophysiology of PE and its impact on alveolar dead space (Figure 2.2). The rationale for each of the steps in the best practice advice was supported by the most current evidence. The benefits and risks of each diagnostic test are summarized in a table (Table 2.2). Additionally, a user-friendly, comprehensive table compared and contrasted all the validated clinical decision rules (Table 2.1). Emerging diagnostic tools, including the use of end tidal carbon dioxide monitoring, also were included. The first manuscript (Chapter 2) was recently accepted for publication in the Journal of Vascular Nursing and is currently in press. In summary, this manuscript provided not only a useful guide for clinicians, but also a clinical context and pathophysiologic foundation for the dissertation research project.

The first study (Chapter Four), End-tidal Carbon Dioxide in the Diagnosis of Acute Pulmonary Embolism in Hospitalized Adult Patients, examined the accuracy, financial impact, and potential risk avoidance of adding $\mathrm{EtCO}_{2}$ to the $\mathrm{PE}$ diagnostic process in hemodynamically stable hospitalized adults at a large, tertiary care, academic medical center in the Midwest. A prospective, descriptive, correlational design 
confirmed that $\mathrm{EtCO}_{2}$ was significantly lower for hospitalized PE positive compared to $\mathrm{PE}$ negative adults. Moreover, an absolute $\mathrm{EtCO}_{2}$ value of $42 \mathrm{mmHg}$ had $100 \%$ sensitivity and $100 \%$ negative predictive value. Importantly, use of this $\mathrm{EtCO}_{2}$ cutoff value did not miss any PE positive participants and could have eliminated approximately $10 \%$ of the CTPA scans. Even with the modest elimination of $10 \%$ of the CTPA scans, an annual estimated cost savings (billable charge) for the study institution was noted to be $\$ 87,947$. In summary, the findings from this study suggest adding $\mathrm{EtCO}_{2}$ assessment to the PE diagnostic process may help the clinician to safely eliminate the need for CTPA scan to rule out PE in up to $10 \%$ of hemodynamically stable hospitalized adults. Integrating this inexpensive, clinically available, non-invasive, and user-friendly technology into the existing BPA could help to eliminate unnecessary imaging, decrease healthcare costs and minimize risks of iatrogenic injury associated with diagnostic imaging.

The second study (Chapter Five), Adherence with American College of Physician's Best Practice Advice for the Diagnosis of Pulmonary Embolism (BPA), evaluated clinician adherence to the 2015 best practice advice for PE diagnosis in hospitalized adults at a large, tertiary care, academic medical center in the Midwest. We found that adherence to the overall BPA guideline was essentially zero. Even though partial adherence was achieved for select aspects of individual BPA statements, their rate of adherence was very low $(\leq 10 \%)$. Moreover, documented adherence with the use of the Pulmonary Embolism Rule-Out Criteria (PERC) in low PE risk individuals also was 
nonexistent. Similarly, age-adjustment of D-dimer results also was not recorded. Collectively, these findings support prior studies that CTPA is the initial diagnostic test in the majority of patients being evaluated for PE in the ED setting but our study expands these findings to the population of hemodynamically stable, hospitalized patients suspected of PE.

Reducing waste in healthcare is an important strategy to decrease healthcare costs and improve healthcare quality and patient safety (Morgan, Wright, \& Dhruva, 2015). Overuse of diagnostic imaging, such as CTPA, is one example of waste in healthcare spending that can adversely impact patient safety due to iatrogenic injury. Both studies in this dissertation examined the PE diagnostic process. Study one's goal was to validate a safe $\mathrm{EtCO}_{2}$ cutoff value for PE exclusion in an effort to eliminate unnecessary radiologic imaging; whereas study two's goal was to evaluate adherence to most current BPA for the PE diagnostic process specifically in the hospitalized adult patient population. While we did find an opportunity to eliminate up to $10 \%$ of unnecessary CTPA imaging with the use of $\mathrm{EtCO}_{2}$ in study one, study two uncovered not only very poor adherence to the current BPA, but also raised several important questions about the BPA. For example, study two findings raises the question of whether the PERC criteria and/or D-dimer testing should be included as a required part of the BPA for hospitalized adults who typically have multiple comorbidities which may make the PERC and Ddimer testing uninformative. Collectively, the results of these two studies are complementary. Adding $\mathrm{EtCO}_{2}$ to the current BPA may help to overcome the fact that the 
PERC evaluation and D-dimer may have limited utility in hospitalized adults. $\mathrm{EtCO}_{2}$ may provide a validated diagnostic tool to help eliminate unnecessary imaging. Nonetheless, while adding $\mathrm{EtCO}_{2}$ to the PE diagnostic process may eliminate healthcare waste and reduce risk of iatrogenic injury associated with overuse of diagnostic imaging, the potential benefits would only be realized if clinicians actually adhere to a future, revised BPA.

\section{Significance of the Dissertation Work}

This dissertation work is significant for a number of reasons. Chapter 4 was the second study to evaluate the use of $\mathrm{EtCO}_{2}$ in hospitalized adults and the first to do so in a heterogeneous sample of hemodynamically stable adults from a combination of general medical-surgical and intensive care units. The findings from Chapter 4 added to the existing body of knowledge and confirmed that $\mathrm{EtCO}_{2}$ is significantly lower in hospitalized PE positive compared to PE negative patients. More importantly, Chapter 4 was able to identify an $\mathrm{EtCO}_{2}$ cutoff score that eliminated the diagnosis of PE in hemodynamically stable hospitalized adults with a $100 \%$ negative predictive value. Moreover, our $\mathrm{EtCO}_{2}$ cutoff value $(\geq 42 \mathrm{mmHg})$ was similar to the only other study of hospitalized patients (Ramme et al., 2016) that evaluated $\mathrm{EtCO}_{2}$ in post-operative orthopedic patients that also found a higher cutoff value $(43 \mathrm{mmHg})$ than previously published by EtCO2 studies with samples of only ED patients or mixed samples of ED and hospitalized patients (Hemnes et al., 2010; Riaz \& Jacob, 2014; Rumpf, Krizmaric, \& 
Grmec, 2009). Indeed, our findings suggest that a higher $\mathrm{EtCO}_{2}$ cutoff value may need to be utilized in hospitalized adults than in adults in the ED.

Our findings also suggest that $\mathrm{EtCO}_{2}$ can safely eliminate up to $10 \%$ of unnecessary CTPA scans to rule out PE in hospitalized adults; thus eliminating unnecessary patient risks and healthcare costs associated with imaging. We found that we needed to test 6 patients being evaluated for acute $\mathrm{PE}$ with $\mathrm{EtCO}_{2}$ in order to eliminate one unnecessary CTPA scan. Skyrocketing healthcare costs are, in part, due to the overuse of diagnostic testing (Morgan et al., 2015). While offering a modest cost savings to the study site, when multiplied across all the hospitals across the nation, the financial impact could be significant. Moreover, the impact on the individual patient cannot be devalued. Avoiding unnecessary radiologic testing has an important physiologic impact on each patient and decreases their risk of allergic reaction, acute kidney injury, and cumulative radiation exposure risks.

To our knowledge, Chapter 5 was the first study to evaluate adherence to the 2015 American College of Physicians Best Practice Advice for the Diagnosis of Pulmonary Embolism. Moreover, this also was the first study to evaluate a PE guideline using a heterogeneous sample of hospitalized adults. The study added to the body of evidence indicating that CTPA is often the first test utilized in the diagnosis of PE (Chen, Gray, Bandiera, MacKinnon, \& Deva, 2015; Tung et al., 2017). A lack of guideline adherence leads to unnecessary testing, specifically for PE it means more CTPA (Yin et al., 2012). 
The Chapter 5 study provided information regarding adherence to and limitations of the BPA for an all hospitalized adult population. Specifically, both the PERC and Ddimer testing were aspects of the BPA where adherence was lacking. Clinical data abstracted from the EMR suggest that both of these assessments would have been positive, had the BPA been followed, begging the question of the utility of these assessments in the hospitalized adults. Thus, our findings suggest that pieces of the BPA may not be appropriate for the inpatient population. Moreover, the collective findings from this dissertation suggest that adding $\mathrm{EtCO}_{2}$ assessment to the PE diagnostic process could not only improve the utility of the BPA for hospitalized patients but also eliminate costly and unnecessary CTPA scans in a portion of patients being evaluated for acute PE.

\section{Challenges \& Limitations}

Research is not without challenges. Knowing that I wanted to do a prospective study comparing $\mathrm{EtCO}_{2}$ to CTPA results in my home institution, I began discussing my future dissertation study long before approval of my proposal to assure study feasibility. All areas from which participants could be recruited were aware of the planned study and had provided verbal support. After obtaining dissertation committee approval, University Of Missouri Institution Review Board approval and study site Research Review Board approval, the PI began notifying all departments of the plan to begin participant recruitment and data collection. Unfortunately, due to organizational and leadership changes in the Emergency Department (ED), the ED no longer allowed PIs to directly recruit participants and/or collect data from the ED patients unless the PI had research 
funding to pay the ED Research Core research nurses for their time to do recruitment and data collection. This revelation was particularly concerning because it was anticipated that the vast majority of study participants would be recruited from the ED rather than the inpatient units. In addition, the use of the ED research nurses for participant recruitment and data collection would require additional human subjects approval from the university affiliated with the study site. Although the PI had previously obtained a small, seed grant from (\$500) from the Society of Critical Care Medicine Shark Bait competition, this amount was insufficient to pay the ED research nurses. At the suggestion of the ED Director of Research, the PI and her advisor wrote and submitted a Just-In- Time (JIT) Grant (\$5000) through the Institute of Clinical and Translational Science at Washington University at St. Louis.

While waiting to hear the funding status of the JIT grant, the PI began participant recruitment and data collection of hospitalized patients. Fortunately, the PI was greeted with an enthusiastic inpatient radiology technician group who were eager to help identify potential participants. During this same time frame, the PI also applied for the Sinclair School of Nursing Jim and Toni Sullivan Endowed Research Grant for PhD Students. Ultimately, the JIT grant was not funded which meant that recruitment and data collection from ED patients would not be possible.

However, subject recruitment of hospitalized patients was steady and exceeded the number originally anticipated from the inpatient setting. With the approval of my committee, I was able to proceed with my dissertation study as planned - just with a 
sample of entirely hospitalized patients. Further good news came when I was awarded the Sullivan Grant (\$1000). Funding from the Society of Critical Care Medicine Shark Bait Competition along with the Sullivan Grant was sufficient to pay for the $\mathrm{EtCO}_{2}$ cannulas needed for the study.

As a result of these aforementioned challenges, there are important limitations to this dissertation work. First, this was a single center study that only included hospitalized adults patients which limits the generalizability of the results. These results may not apply to non-inpatient settings where the $\mathrm{EtCO}_{2}$ cutoff and adherence challenges may be different. However, the results remain valuable as this was the first study to measure $\mathrm{EtCO}_{2}$ and examine adherence to the $2015 \mathrm{BPA}$ in a heterogeneous sample of hospitalized adults suspected of PE. Another limitation was the dissertation work relied on documentation in the EMR for all demographic and clinical information. Documentation may not represent the entirety of a clinician's thought processes; thus, limiting conclusions that can be made related to reasons for ordering the CTPA scan to rule out PE and whether or not any CDR was used (but not documented). Additional variables relevant to BPA adherence (e.g. educational training and years of experience of ordering clinicians) were not examined in this study. Future studies may want to consider interviewing clinicians to gain a better understanding of their diagnostic thought processes, education preparation, and years of experiences with diagnosing PE. 


\section{Future Directions}

My professional plans following completion of the $\mathrm{PhD}$ include continuing to support clinical practice by generating new knowledge through research. I will take on the title of Research Scientist in the Department of Research for Patient Care Services at Barnes-Jewish Hospital. This new role places me in a position to mentor others in clinical research, undertake research in areas to support clinical practice at the hospital, partner with Washington University in inter-professional research and pursue my own clinical research.

Research plans related to this study include seeking grant funding to pursue a larger study to further validate the use of $\mathrm{EtCO}_{2}$ in the diagnosis of hospitalized patients. The opportunity exists to complete a larger inpatient single center prospective study at the study facility and possibly to partner with other hospitals in the Barnes Jewish Christian health care system. There is additional opportunity to update the meta-analysis on $\mathrm{EtCO}_{2}$ that I began in the $\mathrm{PhD}$ program. New studies have been published that need to be included to the analysis. Furthermore, I have developed a relationship with Dr. Ann Hemnes who is willing to share the data she has on the 182 inpatients (Hemnes et al., 2010) for separate analysis. As part of my dissertation research, I collected data related to the three versions of the Wells CDR (traditional, Wells4, simplified Wells). Since examining the accuracy, sensitivity and specificity of the different versions of the Wells CDR was not tied to the specific aims of the dissertation, it will be analyzed after graduation and published as a brief report. Additionally, given my background as a CNS, 
I would like to help effectively implement a revised BPA that incorporates the use of $\mathrm{EtCO}_{2}$ into my health care facility. In particular, it will be essential to incorporate clinical decision support into the EMR and to delve into the clinician perspective help assure that adoption and use of BPA will be realized. 


\section{COMPREHENSIVE REFERENCES}

Ageno, W., Squizzato, A., Wells, P. S., Büller, H. R., \& Johnson, G. (2013). The diagnosis of symptomatic recurrent pulmonary embolism and deep vein thrombosis: guidance from the SSC of the ISTH. Journal of Thrombosis and Haemostasis, 11(8), 1597-1602. https://doi.org/10.1111/jth.12301

Alhassan, S., Sayf, A. A., Arsene, C., \& Krayem, H. (2016). Suboptimal implementation of diagnostic algorithms and overuse of computed tomography-pulmonary angiography in patients with suspected pulmonary embolism. Annals of Thoracic Medicine, 11(4), 254-260. https://doi.org/10.4103/1817-1737.191875

Alkhorayef, M., Babikir, E., Alrushoud, A., Al-Mohammed, H., \& Sulieman, A. (2017). Patient radiation biological risk in computed tomography angiography procedure. Saudi Journal of Biological Sciences, 24(2), 235-240. https://doi.org/10.1016/j.sjbs.2016.01.011

Aschermann, M., \& Widimský, J. (2015). Comparison of ESC Guidelines 2008 and 2014 - Diagnostic and treatment of acute pulmonary embolism. Cor et Vasa, 57(4), e270-e274. https://doi.org/10.1016/j.crvasa.2015.05.013

Bass, A., Fields, K., Goto, R., Turissini, G., Dey, S., \& Russell, L. (2017). Clinical decision rules for pulmonary embolism in hospitalized patients: a systematic literature review and meta-analysis. Thrombosis and Haemostasis, 117(11), 2176-2185. doi:10.1160/TH17-06-0395 
Beckman, M. G., Hooper, W. C., Critchley, S. E., \& Ortel, T. L. (2010). Venous thromboembolism: a public health concern. American Journal of Preventive Medicine, 38(4 Suppl), S495-501. https://doi.org/10.1016/j.amepre.2009.12.017

Bickley, D., Lindsey, J., Saint-Hilaire, R., Hussain, A., Vu, M., MacMahon, H., ... Walter, J. (2015). 678: an analysis of the use of CT pulmonary angiography in young women in an urban emergency department. Critical Care Medicine, 43, 171. https://doi.org/10.1097/01.ccm.0000474506.44733.8f

Brenner, D. J. (2012). Radiation and chest CT scans. Chest, 142(3), 549-550. https://doi.org/10.1378/chest.12-0490

Bryant-Lukosius, D., Carter, N., Reid, K., Donald, F., Martin-Misener, R., Kilpatrick, K., ... DiCenso, A. (2015). The clinical effectiveness and cost-effectiveness of clinical nurse specialist-led hospital to home transitional care: a systematic review: CNS-led transitional care. Journal of Evaluation in Clinical Practice, 21(5), 763-781. doi:10.1111/jep.12401

Chandra, S., Sarkar, P. K., Chandra, D., Ginsberg, N. E., \& Cohen, R. I. (2013). Finding an alternative diagnosis does not justify increased use of CT-pulmonary angiography. BMC Pulmonary Medicine, 13(1), 1-8. https://doi.org/doi:10.1186/1471-2466-13-9

Chen, Y. A., Gray, B. G., Bandiera, G., MacKinnon, D., \& Deva, D. P. (2015). Variation in the utilization and positivity rates of CT pulmonary angiography among 
emergency physicians at a tertiary academic emergency department. Emergency Radiology, 22(3), 221-229. https://doi.org/10.1007/s10140-014-1265-6

Chopra, N., Doddamreddy, P., Grewal, \& Kumar. (2012). An elevated D-dimer value: A burden on our patients and hospitals. International Journal of General Medicine, 87-92. https://doi.org/10.2147/IJGM.S25027

Co, I., Eilbert, W., \& Chiganos, T. (2017). New Electrocardiographic Changes in Patients Diagnosed with Pulmonary Embolism. The Journal of Emergency Medicine, 52(3), 280-285. https://doi.org/10.1016/j.jemermed.2016.09.009

Crawford, F., Andras, A., Welch, K., Sheares, K., Keeling, D., \& Chappell, F. M. (2016). D-dimer test for excluding the diagnosis of pulmonary embolism. Cochrane Database of Systematic Reviews. https://doi.org/10.1002/14651858.CD010864.pub2

Dainiak, N. (2013). Radiation dose and stochastic risk from exposure to medical imaging. CHEST Journal, 144(5), 1431-1433. https://doi.org/10.1378/chest.13-1064

Derby, K. M., Hartung, N. A., Wolf, S. L., Zak, H. L., \& Evenson, L. K. (2017). Clinical Nurse Specialist-Driven Practice Change: Standardizing Vital Sign Monitoring. Clinical Nurse Specialist, 31(6), 343-348. doi:10.1097/NUR.0000000000000330

Dogan, H., de Roos, A., Geleijins, J., Huisman, M., \& Kroft, L. (2015). The role of computed tomography in the diagnosis of acute and chronic pulmonary embolism. 
Diagnostic and Interventional Radiology, 21(4), 307-316. https://doi.org/10.5152/dir.2015.14403

Douma, R. A., Mos, I. C., Erkens, P. M., Nizet, T. A., Durian, M. F., Hovens, M. M., ... Huisman, M. V. (2011). Performance of 4 clinical decision rules in the diagnostic management of acute pulmonary embolism: A prospective cohort study. Annals of Internal Medicine, 154(11), 709-718. https://doi.org/10.7326/0003-4819-154-11201106070-00002

Dybowska, M., Tomkowski, W. Z., Kuca, P., Ubysz, R., Jóźwik, A., \& Chmielewski, D. (2015). Analysis of the accuracy of the Wells scale in assessing the probability of lower limb deep vein thrombosis in primary care patients practice. Thrombosis Journal, 13(1), 1-5. https://doi.org/10.1186/s12959-015-0050-4

Fabius, T. M., Eijsvogel, M. M., van der Lee, I., Brusse-Keizer, M. G. J., \& de Jongh, F. H. (2016). Volumetric capnography in the exclusion of pulmonary embolism at the emergency department: a pilot study. Journal of Breath Research, 10(4), 046016. https://doi.org/10.1088/1752-7163/10/4/046016

Fesmire, F. M., Brown, M. D., Espinosa, J. A., Shih, R. D., Silvers, S. M., Wolf, S. J., \& Decker, W. W. (2011). Critical issues in the evaluation and management of adult patients presenting to the emergency department with suspected pulmonary embolism. Annals of Emergency Medicine, 57(6), 628-652.e75. https://doi.org/10.1016/j.annemergmed.2011.01.020 
Fields, J. M., Davis, J., Girson, L., Au, A., Potts, J., Morgan, C. J., ... Riesenberg, L. A. (2017). Transthoracic echocardiography for diagnosing pulmonary embolism: A systematic review and meta-analysis. Journal of the American Society of Echocardiography, 30(7), 714-723.e4. https://doi.org/10.1016/j.echo.2017.03.004

Freeman, L. M. (2007). Don't bury the v/q scan: it's as good as multidetector CT angiograms with a lot less radiation exposure. Journal of Nuclear Medicine, 49(1), 5-8. https://doi.org/10.2967/jnumed.107.048066

Gibson NS, Sohne M, Kruip MJ, Tick LW, Gerdes VE, Bossuyt PM, Wells PS, \& Buller HR. (2008). Further validation and simplification of the Wells clinical decision rule in pulmonary embolism. Thrombosis Haemostasis. 99(1):229-34. doi: 10.1160/TH07-05-0321.

Gottumukkala, R. V., Glover, M., Yun, B. J., Sonis, J. D., Kalra, M. K., Otrakji, A., ... Prabhakar, A. M. (2017). Allergic-like contrast reactions in the ED: Incidence, management, and impact on patient disposition. The American Journal of Emergency Medicine. https://doi.org/10.1016/j.ajem.2017.10.032

Hemnes, A. R., Newman, A. L., Rosenbaum, B., Barrett, T. W., Zhou, C., Rice, T. W., \& Newman, J. H. (2010). Bedside end-tidal CO2 tension as a screening tool to exclude pulmonary embolism. European Respiratory Journal, 35(4), 735-741. https://doi.org/10.1183/09031936.00084709 
Hughes, M. J., Stein, P. D., \& Matta, F. (2014). Silent pulmonary embolism in patients with distal deep venous thrombosis: Systematic review. Thrombosis Research, 134(6), 1182-1185. https://doi.org/10.1016/j.thromres.2014.09.036

Huisman, M. V., \& Klok, F. A. (2015). Current challenges in diagnostic imaging of venous thromboembolism. Blood, 126(21), 2376-2382. https://doi.org/10.1182/blood-2015-05-640979

Hunsaker, A. R., Lu, M. T., Goldhaber, S. Z., \& Rybicki, F. J. (2010). Imaging in acute pulmonary embolism with special clinical scenarios. Circulation: Cardiovascular Imaging, 3(4), 491-500. https://doi.org/10.1161/CIRCIMAGING.109.855981

Hunt, J. M., \& Bull, T. M. (2011). Clinical review of pulmonary embolism: Diagnosis, prognosis, and treatment. Medical Clinics of North America, 95(6), 1203-1222. https://doi.org/10.1016/j.mena.2011.08.003

Huppmann, M. V., Johnson, W. B., \& Javitt, M. C. (2010). Radiation risks from exposure to chest computed tomography. Seminars in Ultrasound, CT and MRI, 31(1), 1428. https://doi.org/10.1053/j.sult.2009.09.003

Jiménez, D., Resano, S., Otero, R., Jurkojc, C., Portillo, A. K., Ruiz-Artacho, P., ... Yusen, R. D. (2015). Computerized clinical decision support for suspected PE: Table 1. Thorax, 70(9), 909-911. https://doi.org/10.1136/thoraxjnl-2014-206689 
Jung, J.-W., Kang, H.-R., Kim, M.-H., Lee, W., Min, K.-U., Han, M.-H., \& Cho, S.-H. (2012). Immediate hypersensitivity reaction to gadolinium-based MRI contrast media. Radiology, 264(2), 414-422. https://doi.org/10.1148/radiol.12112025

Kabrhel, C., Mark Courtney, D., Camargo, C. A., Plewa, M. C., Nordenholz, K. E., Moore, C. L., ... Kline, J. A. (2010). Factors associated with positive d-dimer results in patients evaluated for pulmonary embolism: factors associated with positive d-dimers. Academic Emergency Medicine, 17(6), 589-597. doi:10.1111/j.1553-2712.2010.00765.x

Kahn, S. R., Houweling, A. H., Granton, J., Rudski, L., Dennie, C., \& Hirsch, A. (2014). Long-term outcomes after pulmonary embolism: current knowledge and future research. Blood Coagulation \& Fibrinolysis, 25(5), 407-415. https://doi.org/10.1097/MBC.0000000000000070

Kearon, C., Akl, E. A., Ornelas, J., Blaivas, A., Jimenez, D., Bounameaux, H., ... Moores, C. L. K. (2016). Antithrombotic therapy for VTE disease. Chest, 149(2), 315-352. https://doi.org/10.1016/j.chest.2015.11.026

Kelly, A.M., Keijers, G., Klim, S., Graham, C., Craig, S., Kuan, W.S. ... Laribi, S. (2017). An observational study of dyspnea in emergency departments: The Asia, Australia, and New Zealand dyspnea in emergency department study (AANZDEM). Academic Emergency Medicine, 24, 328-336. doi: 10.1111/acem.13118. 
Kline, J. A., Hogg, M. M., Courtney, D. M., Miller, C. D., Jones, A. E., \& Smithline, H. A. (2012). D-dimer threshold increase with pretest probability unlikely for pulmonary embolism to decrease unnecessary computerized tomographic pulmonary angiography: Elevated D-dimer threshold. Journal of Thrombosis and Haemostasis, 10(4), 572-581. https://doi.org/10.1111/j.1538-7836.2012.04647.x

Kline, J. A., \& Kabrhel, C. (2015). Emergency evaluation for pulmonary embolism, part 2: Diagnostic approach. The Journal of Emergency Medicine, 49(1), 104-117. https://doi.org/10.1016/j.jemermed.2014.12.041

Kline, J. A., Mitchell, A. M., Kabrhel, C., Richman, P. B., \& Courtney, D. M. (2004). Clinical criteria to prevent unnecessary diagnostic testing in emergency department patients with suspected pulmonary embolism. Journal of Thrombosis and Haemostasis, 2(8), 1247-1255. https://doi.org/10.1111/j.15387836.2004.00790.x

Kline, J. A., Peterson, C. E., \& Steuerwald, M. T. (2010). Prospective evaluation of realtime use of the pulmonary embolism rule-out criteria in an academic emergency department: perc. Academic Emergency Medicine, 17(9), 1016-1019. https://doi.org/10.1111/j.1553-2712.2010.00826.x

Kline, J. A., Webb, W. B., Jones, A. E., \& Hernandez-Nino, J. (2004). Impact of a rapid rule-out protocol for pulmonary embolism on the rate of screening, missed cases, 
and pulmonary vascular imaging in an urban US emergency department. Annals of Emergency Medicine, 44(5), 490-502

Klok, F. A., Dzikowska-Diduch, O., Kostrubiec, M., Vliegen, H. W., Pruszczyk, P., Hasenfuß, G., ... Lankeit, M. (2016). Derivation of a clinical prediction score for chronic thromboembolic pulmonary hypertension after acute pulmonary embolism. Journal of Thrombosis and Haemostasis, 14(1), 121-128. https://doi.org/10.1111/jth.13175

Klok, F.A., Mos, I.C.M. , Nijkeuter, M., Righini, M., Perrier, A., Le Gal, G., \& Huisman, M.V., (2008). Simplification of the revised geneva score for assessing clinical probability of pulmonary embolism.Archives of Internal Medicine, 168(19):2131-2136

Konstantinides, S. V., Torbicki, A., Agnelli, G., Danchin, N., Fitzmaurice, D., Galiè, N., ... Spyropoulos, A. C. (2014). 2014 ESC Guidelines on the diagnosis and management of acute pulmonary embolism. European Heart Journal, 35(43), 3033-3080. https://doi.org/10.1093/eurheartj/ehu283

Kooiman, J., Pasha, S. M., Zondag, W., Sijpkens, Y. W. J., van der Molen, A. J., Huisman, M. V., \& Dekkers, O. M. (2012). Meta-analysis: Serum creatinine changes following contrast enhanced CT imaging. European Journal of Radiology, 81(10), 2554-2561. https://doi.org/10.1016/j.ejrad.2011.11.020 
Le Gal, G., Righini, M., Roy, P.M., Sanchez, O., Aujesky, D., Bounameaux, H.,\& Perrier, A. (2006). Prediction of pulmonary embolism in the emergency department: The revised geneva score. Annals of Internal Medicine, ;144:165171.

LaMori, J. C., Shoheiber, O., Mody, S. H., \& Bookhart, B. K. (2015). Inpatient resource use and cost burden of deep vein thrombosis and pulmonary embolism in the United States. Clinical Therapeutics, 37(1), 62-70. https://doi.org/10.1016/j.clinthera.2014.10.024

Lankeit, M., GÃṛmez, V., Wagner, C., Aujesky, D., Recio, Mã., Briongos, S., ... JimÃ $\breve{S} n e z$, D. (2012). A strategy combining imaging and laboratory biomarkers in comparison with a simplified clinical score for risk stratification of patients with acute pulmonary embolism. CHEST Journal, 141(4), 916-922.

Lapner, S. T., \& Kearon, C. (2013). Diagnosis and management of pulmonary embolism. BMJ, 346, f757. https://doi.org/10.1136/bmj.f757

Lavorini, F., Di Bello, V., De Rimini, M. L., Lucignani, G., Marconi, L., Palareti, G., ... Pistolesi, M. (2013). Diagnosis and treatment of pulmonary embolism: A multidisciplinary approach. Multidisciplinary Respiratory Medicine, 8(1), 1. https://doi.org/10.1186/2049-6958-8-75

Le Roux, P.-Y., Palard, X., Robin, P., Delluc, A., Abgral, R., Querellou, S., ... Salaun, P.-Y. (2014). Safety of ventilation/perfusion single photon emission computed 
tomography for pulmonary embolism diagnosis. European Journal of Nuclear Medicine and Molecular Imaging, 41(10), 1957-1964. https://doi.org/10.1007/s00259-014-2763-1

Levin, D., Seo, J. B., Kiely, D. G., Hatabu, H., Gefter, W., van Beek, E. J. R., \& Schiebler, M. L. (2015). Triage for suspected acute pulmonary embolism: Think before opening Pandora's Box. European Journal of Radiology, 84(6), 12021211. https://doi.org/10.1016/j.ejrad.2015.03.023

Lippi, G., Danese, E., Favaloro, E., Montagnana, M., \& Franchini, M. (2015). Diagnostics in venous thromboembolism: from origin to future prospects. Seminars in Thrombosis and Hemostasis, 41(04), 374-381. https://doi.org/10.1055/s-0034-1544003

Long, B., \& Koyfman, A. (2016). Best clinical practice: current controversies in pulmonary embolism imaging and treatment of subsegmental thromboembolic disease. The Journal of Emergency Medicine. Retrieved from http://www.sciencedirect.com/science/article/pii/S0736467916306801

Lucassen, W., Geersing, G.-J., Erkens, P. M., Reitsma, J. B., Moons, K. G., Büller, H., \& van Weert, H. C. (2011). Clinical decision rules for excluding pulmonary embolism: a meta-analysis. Annals of Internal Medicine, 155(7), 448-460.

Ma, Y., Wang, Y., Liu, D., Ning, Z., An, M., Wu, Q., \& Lin, Y. (2017). A safe strategy to rule out pulmonary embolism: The combination of the Wells score and D-dimer 
test: One prospective study. Thrombosis Research, 156, 160-162.

https://doi.org/10.1016/j.thromres.2017.06.018

Ma, Y., Yan, S., Zhou, L., \& Yuan, D.-T. (2016). Competitive assessments of pulmonary embolism: Noninvasiveness versus the golden standard. Vascular, 24(2), 217224. https://doi.org/10.1177/1708538115589893

Manara, A., D'hoore, W., \& Thys, F. (2013). Capnography as a diagnostic tool for pulmonary embolism: A meta-analysis. Annals of Emergency Medicine, 62(6), 584-591. https://doi.org/10.1016/j.annemergmed.2013.04.010

Marshall, P. S., Mathews, K. S., \& Siegel, M. D. (2011). Diagnosis and management of life-threatening pulmonary embolism. Journal of Intensive Care Medicine, 26(5), 275-294. https://doi.org/10.1177/0885066610392658

Meinel, F. G., Nance, J. W., Schoepf, U. J., Hoffmann, V. S., Thierfelder, K. M., Costello, P., ... Bamberg, F. (2015). Predictive value of computed tomography in acute pulmonary embolism: Systematic review and meta-analysis. The American Journal of Medicine, 128(7), 747-759.e2. doi:10.1016/j.amjmed.2015.01.023

McConnell, M. V., Solomon, S. D., Rayan, M., Come, P., Goldhaber, S., \& Lee, R. (1996). Regional right ventricular dysfunction detected by echocardiography in acute pulmonary embolism. American Journal of Cardiology, 78, 469-473. https://doi.org/10.1016/S0002-9149(96)00339-6 
McDonald, J. S., McDonald, R. J., Carter, R. E., Katzberg, R. W., Kallmes, D. F., \& Williamson, E. E. (2014). Risk of intravenous contrast material-mediated acute kidney injury: A propensity score-matched study stratified by baseline-estimated glomerular filtration rate. Radiology, 271(1), 65-73. https://doi.org/10.1148/radiol.13130775

Montagnana, M., Cervellin, G., Franchini, M., \& Lippi, G. (2011). Pathophysiology, clinics and diagnostics of non-thrombotic pulmonary embolism. Journal of Thrombosis and Thrombolysis, 31(4), 436-444. https://doi.org/10.1007/s11239010-0519-8

Moores, L. K. (2011). Current approach to the diagnosis of acute nonmassive pulmonary embolism. CHEST Journal, 140(2), 509. https://doi.org/10.1378/chest.10-2468

Nazerian, P., Vanni, S., Volpicelli, G., Gigli, C., Zanobetti, M., Bartolucci, M., ... Grifoni, S. (2014). Accuracy of point-of-care multiorgan ultrasonography for the diagnosis of pulmonary embolism. CHEST Journal, 145(5), 950-957. https://doi.org/10.1378/chest.13-1087

Nazerian, P., Volpicelli, G., Gigli, C., Becattini, C., Sferrazza Papa, G. F., Grifoni, S., ... the Ultrasound Wells Study Group. (2017). Diagnostic performance of wells score combined with point-of-care lung and venous ultrasound in suspected pulmonary embolism. Academic Emergency Medicine, 24(3), 270-280.

https://doi.org/10.1111/acem.13130 
Newnham, M., Stone, H., Summerfield, R., \& Mustfa, N. (2013). Performance of algorithms and pre-test probability scores is often overlooked in the diagnosis of pulmonary embolism. British Medical Journal, 346(mar19 2), f1557-f1557. https://doi.org/10.1136/bmj.f1557

Nordenholz, K. E., Naviaux, N. W., Stegelmeier, K., Haukoos, J. S., Wolf, S. J., McCubbin, T., \& Heard, K. (2007). Pulmonary embolism risk assessment screening tools: the interrater reliability of their criteria. The American Journal of Emergency Medicine, 25(3), 285-290. https://doi.org/10.1016/j.ajem.2006.08.016

Omar, H. R., Mirsaeidi, M., Weinstock, M. B., Enten, G., Mangar, D., \& Camporesi, E. M. (2017). Syncope on presentation is a surrogate for submassive and massive acute pulmonary embolism. The American Journal of Emergency Medicine. https://doi.org/10.1016/j.ajem.2017.11.014

Oqab, Z., Ganshorn, H., \& Sheldon, R. (2017). Prevalence of pulmonary embolism in patients presenting with syncope. A systematic review and meta-analysis. The American Journal of Emergency Medicine. https://doi.org/10.1016/j.ajem.2017.09.015

Parikh, N., Morris, E., Babb, J., Wickstrom, M., McMenamy, J., Sharma, R., ... Kim, D. (2015). MDCT diagnosis of acute pulmonary embolism in the emergent setting. Emergency Radiology, 22(4), 379-384. https://doi.org/10.1007/s10140-014-1290- 
Perelas, A., Dimou, A., Saenz, A., Rhee, J. H., Teerapuncharoen, K., Rowden, A., \& Eiger, G. (2015). CT pulmonary angiography utilization in the emergency department: Diagnostic yield and adherence to current guidelines. American Journal of Medical Quality, 30(6), 571-577. https://doi.org/10.1177/1062860614543302

Perelas, A., Dimou, A., Saenz, A., Rhee, J. H., Teerapuncharoen, K., Rowden, A., \& Eiger, G. (2015b). Incidental findings on computed tomography angiography in patients evaluated for pulmonary embolism. Annals of the American Thoracic Society, 12(5), 689-695. doi:10.1513/AnnalsATS.201404-144OC

Perera, M., Aggarwal, L., Scott, I. A., \& Cocks, N. (2017). Underuse of risk assessment and overuse of CTPA in patients with suspected pulmonary thromboembolism: Overuse of CTPA in suspected PTE. Internal Medicine Journal. https://doi.org/10.1111/imj.13524

Piovella, F., \& Iosub, D. I. (2016). Acute pulmonary embolism: risk assessment, risk stratification and treatment options: Acute pulmonary embolism. The Clinical Respiratory Journal, 10(5), 545-554. https://doi.org/10.1111/crj.12264

Planquette, B., Ferré, A., Peron, J., Vial-Dupuy, A., Pastre, J., Mourin, G., ... Sanchez, O. (2016). Residual pulmonary vascular obstruction and recurrence after acute pulmonary embolism. A single center cohort study. Thrombosis Research, 148, 70-75. https://doi.org/10.1016/j.thromres.2016.10.030 
Prentice, D. and Wipke-Tevis, D. D. (2018). Diagnosis of pulmonary embolism: Following the evidence from suspicion to certainty, Journal of Vascular Nursing, In Press

Prentice, D. (2014). Three capnography methods for the diagnosis of pulmonary embolism, Critical Care Medicine, 42, A1528, DOI:

10.1097/01.ccm.0000458194.72927.ef.

Puurunen, M. K., Gona, P. N., Larson, M. G., Murabito, J. M., Magnani, J. W., \& O’Donnell, C. J. (2016). Epidemiology of venous thromboembolism in the Framingham Heart Study. Thrombosis Research, 145, 27-33. https://doi.org/10.1016/j.thromres.2016.06.033

Raja, A. S., Greenberg, J. O., Qaseem, A., Denberg, T. D., Fitterman, N., \& Schuur, J. D. (2015). Evaluation of patients with suspected acute pulmonary embolism: Best practice advice from the clinical guidelines committee of the American college of physicians. Annals of Internal Medicine, 163(9), 701. https://doi.org/10.7326/M14-1772

Raja, A. S., Ip, I. K., Dunne, R. M., Schuur, J. D., Mills, A. M., \& Khorasani, R. (2015). Effects of performance feedback reports on adherence to evidence-based guidelines in use of CT for evaluation of pulmonary embolism in the emergency department: a randomized trial. American Journal of Roentgenology, 205(5), 936940. doi:10.2214/AJR.15.14677 
Ramme, A. J., Iturrate, E., Dweck, E., Steiger, D. J., Hutzler, L. H., Fang, Y., ... Sigmund, A. E. (2016). End tidal carbon dioxide as a screening tool for computed tomography angiogram in postoperative orthopaedic patients suspected of pulmonary embolism. The Journal of Arthroplasty, 31(10), 2348-2352. https://doi.org/10.1016/j.arth.2016.03.033

Reissig, A., Copetti, R., \& Kroegel, C. (2011). Current role of emergency ultrasound of the chest. Critical Care Medicine, 39(4), 839-845. https://doi.org/10.1097/CCM.0b013e318206d6b8

Riaz, I., \& Jacob, B. (2014). Pulmonary embolism in Bradford, UK: Role of end-tidal CO2 as a screening tool. Clinical Medicine, 14(2), 128-133. https://doi.org/10.7861/clinmedicine.14-2-128

Righini, M., Van Es, J., Den Exter, P. L., Roy, P.-M., Verschuren, F., Ghuysen, A., ... Le Gal, G. (2014). Age-adjusted d-dimer cutoff levels to rule out pulmonary embolism: the adjust-pe study. JAMA, 311(11), 1117. https://doi.org/10.1001/jama.2014.2135

Riley, R. S., Gilbert, A. R., Dalton, J. B., Pai, S., \& McPherson, R. A. (2016). Widely used types and clinical applications of d-dimer assay. Laboratory Medicine, 47(2), 90-102. https://doi.org/10.1093/labmed/lmw001

Rohner, D. J., Bennett, S., Samaratunga, C., Jewell, E. S., Smith, J. P., Gaskill-Shipley, M., \& Lisco, S. J. (2013). Cumulative total effective whole-body radiation dose in 
critically ill patients. Chest, 144(5), 1481-1486. https://doi.org/10.1378/chest.12$\underline{2222}$

Ryan, M. A. (2017). Adherence to Clinical Practice Guidelines. Otolaryngology-Head and Neck Surgery, 157(4), 548-550. doi:10.1177/0194599817718822

Rumpf, T. H., Krizmaric, M., Grmec, S., \& others. (2009). Capnometry in suspected pulmonary embolism with positive D-dimer in the field. Critical Care, 13(6), 1-9. https://doi.org/10.1186/cc8197

Salaun, P.-Y. (2011). Noninvasive diagnosis of pulmonary embolism. CHEST Journal, 139(6), 1294. https://doi.org/10.1378/chest.10-1209

Schembri, G. P., Miller, A. E., \& Smart, R. (2010). Radiation dosimetry and safety issues in the investigation of pulmonary embolism. Seminars in Nuclear Medicine, 40(6), 442-454. https://doi.org/10.1053/j.semnuclmed.2010.07.007

Schouten, H. J., Geersing, G.-J., Oudega, R., van Delden, J. J. M., Moons, K. G. M., \& Koek, H. L. (2014). Accuracy of the wells clinical prediction rule for pulmonary embolism in older ambulatory adults. Journal of the American Geriatrics Society, 62(11), 2136-2141. https://doi.org/10.1111/jgs.13080

Sedighi, I. (2013). Interpretation of diagnostic tests: Likelihood ratio vs predictive value. Iranian Journal of Pediatrics, 23(6), 717. 
Sherk, W. M., \& Stojanovska, J. (2017). Role of clinical decision tools in the diagnosis of pulmonary embolism. American Journal of Roentgenology, 208(3), W60-W70. https://doi.org/10.2214/AJR.16.17206

Singh, B., Mommer, S. K., Erwin, P. J., Mascarenhas, S. S., \& Parsaik, A. K. (2013). Pulmonary embolism rule-out criteria (PERC) in pulmonary embolismrevisited: A systematic review and meta-analysis. Emergency Medicine Journal, 30(9), 701-706. https://doi.org/10.1136/emermed-2012-201730

Smith, S., Geske, J. B., Kathuria, P., Cuttica, M., Schimmel, D. R., Courtney, D. M., ... Wunderink, R. G. (2016). Analysis of national trends in admissions for pulmonary embolism. Chest, 150, 35-45. https://doi.org/10.1016/j.chest.2016.02.638

Smith, S., Geske, J., McNamara, D., Waterer, G., \& Wunderink, R. (2013). Pulmonary embolism trends from 1996 to 2010. Chest, 144(4), 850A. https://doi.org/10.1378/chest.1704692

Solomon, C. G., \& Greer, I. A. (2015). Pregnancy complicated by venous thrombosis. New England Journal of Medicine, 373(6), 540-547. https://doi.org/10.1056/NEJMcp1407434

Stein, P. D., Fowler, S. E., Goodman, L. R., Gottschalk, A., Hales, C. A., Hull, R. D., ... Woodard, P. K. (2006). Multidetector computed tomography for acute pulmonary 
embolism. New England Journal of Medicine, 354(22), 2317-2327. https://doi.org/10.1056/NEJMoa052367

Stein, P. D., Matta, F., Musani, M. H., \& Diaczok, B. (2010). Silent pulmonary embolism in patients with deep venous thrombosis: A systematic review. The American Journal of Medicine, 123(5), 426-431.

https://doi.org/10.1016/j.amjmed.2009.09.037

Stone, J., Hangge, P., Albadawi, H., Wallace, A., Shamoun, F., Knuttien, M. G., ... Oklu, R. (2017). Deep vein thrombosis: Pathogenesis, diagnosis, and medical management. Cardiovascular Diagnosis and Therapy, 7(S3), S276-S284. https://doi.org/10.21037/cdt.2017.09.01

Sverzellati, N., De Filippo, M., Quintavalla, M., Randi, G., Franco, F., Cobelli, R., ... Zompatori, M. (2014). Interobserver reliability of the chest radiograph in pulmonary embolism. Clinical and Applied Thrombosis/Hemostasis, 20(2), 147151. https://doi.org/10.1177/1076029612458969

Traub, S. J., Mitchell, A. M., Jones, A. E., Tang, A., O’Connor, J., Nelson, T., ... Shapiro, N. I. (2013). N-acetylcysteine plus intravenous fluids versus intravenous fluids alone to prevent contrast-induced nephropathy in emergency computed tomography. Annals of Emergency Medicine, 62(5), 511-520.e25. https://doi.org/10.1016/j.annemergmed.2013.04.012 
Tromeur, C., van der Pol, L. M., Klok, F. A., Couturaud, F., \& Huisman, M. V. (2017). Pitfalls in the diagnostic management of pulmonary embolism in pregnancy. Thrombosis Research, 151, S86-S91. https://doi.org/10.1016/S0049$3848(17) 30075-0$

van Es, J., Douma, R. A., Schreuder, S. M., Middeldorp, S., Kamphuisen, P. W., Gerdes, V. E., \& Beenen, L. F. (2013). Clinical impact of findings supporting an alternative diagnosis on CT pulmonary angiography in patients with suspected pulmonary embolism. CHEST Journal, 144(6), 1893-1899.

van Es, N., Kraaijpoel, N., Klok, F. A., Huisman, M. V., Den Exter, P. L., Mos, I. C. M., ... Bossuyt, P. M. (2017). The original and simplified Wells rules and ageadjusted D-dimer testing to rule out pulmonary embolism: An individual patient data meta-analysis. Journal of Thrombosis and Haemostasis, 15(4), 678-684.

van der Hulle, T., Dronkers, C. E. A., Klok, F. A., \& Huisman, M. V. (2016). Recent developments in the diagnosis and treatment of pulmonary embolism. Journal of Internal Medicine, 279(1), 16-29. https://doi.org/10.1111/joim.12404

Walsh, B. M., \& Moore, C. L. (2015). McConnell's sign is not specific for pulmonary embolism: Case report and review of the literature. The Journal of Emergency Medicine, 49(3), 301-304. https://doi.org/10.1016/j.jemermed.2014.12.089

Wang, R. C., Bent, S., Weber, E., Neilson, J., Smith-Bindman, R., \& Fahimi, J. (2016). The impact of clinical decision rules on computed tomography use and yield for 
pulmonary embolism: A systematic review and meta-analysis. Annals of Emergency Medicine, 67(6), 693-701.e3. https://doi.org/10.1016/j.annemergmed.2015.11.005

Weekes, A. J., Johnson, A. K., Troha, D., Thacker, G., Chanler-Berat, J., \& Runyon, M. (2017). Prognostic value of right ventricular dysfunction markers for serious adverse events in acute normotensive pulmonary embolism. The Journal of Emergency Medicine, 52(2), 137-150. https://doi.org/10.1016/j.jemermed.2016.09.002

Wells, P. (1998). Use of a clinical model for safe management of patients with suspected pulmonary embolism. Annals of Internal Medicine, 129(12), 997. https://doi.org/10.7326/0003-4819-129-12-199812150-00002

Wells, P., Anderson, D., Bormanis, J., Guy, F., Mitchell, M., Gray, L., ... Lewandowski, B. (1997). Value of assessment of pretest probability of deep-vein thrombosis in clinical management. The Lancet, 350(9094), 1795-1798. https://doi.org/10.1016/S0140-6736(97)08140-3

Wells, P., Anderson, D., Rodger, M., Ginsberg, J., Kearon, C., Gent, M., ... Hirsh, J. (2000). Derivation of a simple clinical model to categorize patients' probability of pulmonary embolism: Increasing the models utility with the simplified d-dimer. Thrombosis and Haemostasis, 83(03), 416-420. doi:10.1055/s-0037-1613830 
West, J. B., \& Luks, A. (2016). West's Repiratory Physiology: The Essentials (10th ed.). Philadelphia: Wolters Kluwer.

Wicki, J., Perneger, T., Junod, A., Bounameaux, H., \& Perrier, A. (2000). Assessing clinical probability of pulmonary embolism in the emergency ward: A simple score. Journal of General Internal Medicine, 15(s2), 10-10. doi:10.1046/j.15251497.2000.15200-42.x

Woller, S. C., Stevens, S. M., Adams, D. M., Evans, R. S., Lloyd, J. F., Snow, G. L., ... Elliott, C. G. (2014). Assessment of the safety and efficiency of using an ageadjusted d-dimer threshold to exclude suspected pulmonary embolism. Chest, 146(6), 1444-1451. https://doi.org/10.1378/chest.13-2386

Worsley, D. F., \& Alavi, A. (1995). Comprehensive analysis of the results of the pioped study. Journal of Nuclear Medicine, 36(12), 2380-2387.

Yan, Z., Ip, I. K., Raja, A. S., Gupta, A., Kosowsky, J. M., \& Khorasani, R. (2016). Yield of CT pulmonary angiography in the emergency department when providers override evidence-based clinical decision support. Radiology, 282(3), 717-725

Yin, F., Wilson, T., Della Fave, A., Larsen, M., Yoon, J., Nugusie, B., ... Chow, R. D. (2012). Inappropriate use of D-dimer assay and pulmonary CT angiography in the evaluation of suspected acute pulmonary embolism. American Journal of Medical Quality, 27(1), 74-79. https://doi.org/10.1177/1062860611407907 
Youssf, A. R. I., Ismail, M. F. M., ElGhamry, R., \& Reyad, M. R. (2014). Diagnostic accuracy of D-dimer assay in suspected pulmonary embolism patients. Egyptian Journal of Chest Diseases and Tuberculosis, 63(2), 411-417. https://doi.org/10.1016/j.ejcdt.2013.12.015

Zanzonico, P., Rothenberg, L. N., \& Strauss, H. W. (2006). Radiation exposure of computed tomography and direct intracoronary angiography. Journal of the American College of Cardiology, 47(9), 1846-1849. https://doi.org/10.1016/j.jacc.2005.10.075

Zhu, R., \& Ma, X.-C. (2017). Clinical value of ultrasonography in diagnosis of pulmonary embolism in critically ill patients. Journal of Translational Internal Medicine, 5(4). https://doi.org/10.1515/jtim-2017-0034 


\title{
APPENDIX A:
}

\section{University of Missouri Human Subject Approval}

\author{
APPENDIX A
}

University of Missouri Human Subject Approval

방

Institutional Review Board

University of Missouri-Columbia

190 Galena Hall

Columbia, MO 65201

573-882-3181

irb@missouri.edu

March 02, 2018

Principal Investigator: Donna M Prentice, MSN

Department: School of Nursing

Your Amendment Form to project entitled Exhaled Carbon Dioxide Level in the Diagnosis of Acute Pulmonary Embolism in Adult Patients was reviewed and approved by the MU Institutional Review Boadrd according to the terms and conditions described below:

IRB Project Number 2009689

IRB Review Number 235101

Funding Source University of Missouri - Columbia

Initial Application Approval Date November 16, 2017

Approval Date March 02, 2018

IRB Expiration Date November 16, 2018

Level of Review Expedited

Project Status Active - Open to Enrollment

Risk Level Minimal Risk

Type of Consent Consent with Waiver of

Documentation

The principal investigator (PI) is responsible for all aspects and conduct of this study. The PI must comply with the following conditions of the approval:

1. No subjects may be involved in any study procedure prior to the IRB approval date or after the expiration date.

2. All unanticipated problems and deviations must be reported to the IRB within 5 business days.

3. All changes must be IRB approved prior to implementation unless they are intended to reduce immediate risk.

4. All recruitment materials and methods must be approved by the IRB prior to being used.

5. The Continuing Review Report (CRR) must be submitted to the IRB for review and approval at least 30 days prior to the project expiration date. If the study is complete, the Completion/Withdrawal Form may be submitted in lieu of the CRR.

6. Maintain all research records for a period of seven years from the project completion date.

7. Utilize the IRB stamped consent documents and other approved research documents located within the document storage section of eCompliance. These documents are 


\section{APPENDIX B:}

\section{Barnes-Jewish Hospital Approval Letter}

APPENDIX B

Barnes-Jewish Hospital Approval Letter

December 8, 2017

Donna Prentice, RN, MSN $_{(\mathrm{R})}$, ACNS-BC, FCCM

Barnes-Jewish Hospital

One Barnes-Jewish Hospital Plaza

St. Louis, MO 63110

Dear Ms. Prentice,

Thank-you for the submission of your proposal for your doctoral research study titled, "Exhaled carbon dioxide level in the diagnosis of acute pulmonary embolism in adult patients". The committee has approved your proposal as written. However, we have included committee suggestions, as discussed with you in the review meeting.

Study proposal suggestions:

1) Consent form: Clarifying sentence for \#3 in regards to the purpose of comparing CTPA result with $\mathrm{co} 2$ result.

2) Might consider eluding to the potential of false-negatives or instances where an EtCO2 may not increase despite a PE existing, as well as falsepositives or instances where the EtCO2 may be increased but secondary to other processes.

3) May be useful to include "reason for hospital admission" (admitted for PE rule/out vs admitted for non-correlated cause).

4) Suggest re-requesting the data for cost of CTPA since listed cost of $\$ 2,000$ is more than two years old. $\$ 2,000$ seems low for a scan.

5) Consider adding patient BMI to account for possible hypoventilation.

6) Include your mailstop number with your contact information.

Please do not hesitate to contact us with any questions regarding the proposal review. We wish you all the best with your doctoral research.

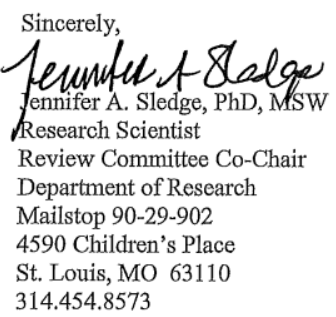

Dynn Schallem

Lynn Schallom, RN, PhD, CCNS, FCCM

Director, Department of Research

Review Committee Co-Chair

Department of Research

Mailstop 90-29-902

4590 Children's Place

St. Louis, MO 63110

314.286.0835

One Barnes-Jewish Hospital Plaza · St. Louis, Missouri 63110-1092 - www.BarnesJewish.org

Barnes-Jewish Hospital at Washington University Medical Center

BQ HealthCare 


\title{
APPENDIX C:
}

\section{Waiver of Documentation of Consent}

\author{
APPENDIX C \\ Consent \\ WAIVER OF DOCUMENTATION OF CONSENT
}

\author{
InVESTigator's Name: DONNa Prentice RN, MSN(R), ACNS-BC, FCCM \\ PROJECT \# 2009689
}

Study Title: Exhaled Carbon Dioxide Level in the Diagnosis of Acute Pulmonary Embolism in Adult Patients

If you are the legally authorized representative of a person who is being invited to participate in this study, the word "you" in this document refers to the person you represent. As the legally authorized representative, you will be asked to read this document to give permission for the person you represent to participate in this research study.

1. I would like to ask you to participate in a study that involves research.

2. Participation is voluntary and your decision not to participate will not involve any penalty or loss of benefits and in no way affect the medical care you receive at Barnes-Jewish Hospital.

If you agree to be in the study, I will place a small, soft plastic tube (cannula) in your nose, similar to what you wear if you are getting oxygen. The cannula will measure the carbon dioxide (air from your lungs) you are breathing out. You will need to be sitting or lying in bed during the measurement. The cannula will be in place for about 10 minutes. If you already have your carbon dioxide measured as part of your routine care, I will write down the carbon dioxide values from the monitor. I also will get information from your medical record about your symptoms (physical complaints) when you came to the hospital as well as the symptoms that led to the order for the radiology test (CT scan of your lungs), vital signs (e.g. blood pressure, heart rate, breathing rate, temperature, oxygen level in your blood), past medical history, current blood test results, location/ service, venous dopplers (an ultrasound or scan looking for blood clots in your arms and/or legs), and the radiology test (CT scan of your lungs) that will tell if you have a blood clot in your lungs.

3. The purpose of our study is to find out if measuring carbon dioxide, the air that comes out of your lungs when you breathe out, can help us determine if people do not have a blood clot (pulmonary embolism) in the blood vessels of their lungs.

4. About 150 people at Barnes-Jewish Hospital will participate in this study.

5. The study staff may remove you from the study at any time after explaining to you the reason for removing you.

6. It is possible that there may be a temporary, minor discomfort from the plastic tube used to measure your carbon dioxide; however, that is not likely. If you have discomfort from the tube, please let the researcher know right away. 
7. If you agree to take part in this study, there are no benefits to you. You may expect to benefit from taking part in this research to the extent that you are adding to healthcare knowledge. We hope what we learn from this study will help prevent people in the future from having an unnecessary CT scan.

8. Being a part of this study will not change the treatment plan that your doctor has prescribed for your care. You do not have to be in this study. It is up to you. If you say OK now but you change your mind later, that is OK too. All you have to do is tell us.

9. When we are done with the study, we will write a report about what we found out. We will not use your name in the report. The research team will only use and share your information as talked about during this consent process. Once the information is de-identified (cannot be linked to you), it may be shared for other purposes not discussed during this consent process. [ie kept confidential.]

10. You will not be charged for being in this study.

11. There is no compensation given to you for being in this study.

12. If you have any questions regarding your rights as a participant in this research and/or concerns about the study, or if you feel under any pressure to enroll or to continue to participate in this study, you may contact the University of Missouri Health Sciences Institutional Review Board (which is a group of people who review the research studies to protect participants' rights) at (573) 882-3181.

13. You may ask more questions about the study at any time. If you have any problems or questions, you may contact Donna Prentice RN, MSN, ACNS-BC the main researcher for the study at (314)-262-2648.

14. A copy of this script will be given to you to keep.

\section{HIPAA CONSENT:}

Purpose: State and federal privacy laws protect the use and release of your health information. If you decide to give your permission to participate in the study you will also be consenting for the HIPAA authorization. This section describes the different ways that the researcher, the study team and the sponsor may use your health information for the research study. Agreeing to this authorization is completely voluntary.

Your authorization applies to the information described below. By law, the information must be limited to the minimum necessary information needed to accomplish the purpose of the research. This information includes information from your medical record about your physical complaints (symptoms) when you came to the hospital and that led to the order of the CT scan, vital signs (e.g. blood pressure, heart rate, breathing rate, temperature, oxygen level in your blood), past medical history, risk of pulmonary embolism score, type of insurance, location/service, current

MU: Waiver of Documentation of Consent

IRB USE ONLY

Approval Date: March 2, 2018

Expiration Date: November 16, 2018 
blood test results, venous dopplers (an ultrasound or scan looking for blood clots in your arms and legs), and the radiology test (CT scan of your lungs) that will tell if you have a blood clot in your lung.

The primary investigator listed at the top and the study team may use and/or disclose your information to the following person(s) or class of persons: Compliance and Safety Monitors, the MU Health Sciences Institutional Review Board, Government agencies.

My personal health information (PHI) will be used and/or disclosed upon request for the following purposes: Publications and presentation that will not identify me, auditing, administrative reviews, study outcomes.

Unless you revoke (take back) your authorization, your authorization will allow us to use and/or disclose your information will expire at the end of the research study.

I understand that $I$ have a right to revoke this authorization at any time. My revocation must be in writing in a letter sent to the Principal Investigator at 4590 Children's Place, St. Louis, Missouri 63110. I am aware that my revocation is not effective to the extent that the persons I have authorized to use and/or disclose my PHI have already acted in reliance upon this authorization.

I understand that my personal health information will only be used as described in this authorization in relation to the research study. I am also aware that if I choose to share the information defined in this authorization to anyone not directly related to this research project, uld no longer protect this information. In addition, I understand that if my personal the law protections under the law, then such information may be redisclosed and would no longer be protected.

I understand that I have a right not to authorize the use and/or disclosure of my personal health information. In such a case I would choose not to agree to this authorization document I understand I will not be able to participate in a research study if I do not do so.

I agree that I will not have a right to access my personal health information obtained or created in the course of the research project until the end of the study.

10. If I have not already received a copy of the University of Missouri Healthcare Privacy Notice, I may request one. If I have any questions or concerns about my privacy rights I should US Privacy Officer at 573-882-9054 or the Campus Privacy Officer at 573-882-9500.

* Study Representative is a person authorized to obtain consent. Per the policies of the University of Misour physicin who is either the Principal or Co-Investigator. If the study is deemed either 'significant ingestigator.

IRB USE ONLY

Approval Date: March 2, 2018

Expiration Date: November 16, 2018 


\section{APPENDIX D}

\section{Diagnosis of PE: Data Collection Tool}

\begin{tabular}{|c|c|}
\hline \multirow{2}{*}{\multicolumn{2}{|c|}{$\begin{array}{l}\text { Subject \# } \\
\text { Date? Time of admission: }\end{array}$}} \\
\hline & \\
\hline Reason for admission: & $\begin{array}{l}\text { Comorbidities (from EHR): HTN, COPD, hyperlipidemia, CAD, DM, Asthma, CHF, CKD, } \\
\text { ESRD, CVA, OSA, CA, Depression/Anxiety, pHTN, GERD/PUD, hypothyroid, SZ }\end{array}$ \\
\hline \multicolumn{2}{|r|}{ ( } \\
\hline gender: $\mathrm{M} F$ & ACP Guidelines for best Practice \\
\hline race: & BP1.Use of validated CDR: yes $\quad$ no \\
\hline BP at presentation: & $\begin{array}{l}\text { BP2. low probability and meet all PERC screen: no further imaging yes no } \\
\text { n/a } \\
\text { And no D-dimer } \quad \text { yes } \quad \text { no n/a }\end{array}$ \\
\hline $\mathrm{BP}$ at $\mathrm{EtCO}_{2}$ measure: & $\begin{array}{l}\text { BP3. intermediate probability CDR or if low probability CDR \& do not meet } \\
\text { PERC: D-dimer obtained }\end{array}$ \\
\hline HR at presentation & $\begin{array}{l}\text { BP4. use of age adjusted D-dimer if }>50 \\
\text { dimer }\end{array}$ \\
\hline $\mathrm{HR}$ at $\mathrm{EtCO}_{2}$ measure: & $\begin{array}{l}\text { BP5. no imaging if D-dimer is normal including use of age adjusted normal; } \\
\begin{array}{cll}\text { Yes } & \text { no } & \text { n/a no dimer }\end{array}\end{array}$ \\
\hline RR at presentation: & $\begin{array}{l}\text { BP6: high probability: no D-dimer yes no n/a } \\
\text { CTPA obtained or V/Q only if CTPA is contraindicated } \\
\text { Yes } \quad \text { no n/a }\end{array}$ \\
\hline $\mathrm{RR}$ at $\mathrm{EtCo}_{2}$ measure: & $\begin{array}{llll}\text { ABG: } & \text { yes } & \text { no } & \text { value }\end{array}$ \\
\hline $\mathrm{SpO}_{2:}$ at presentation & EtCO ${ }_{2}$ Gradient: $\left(\mathrm{PaCO}_{2}-\mathrm{EtCO}_{2}\right)$ \\
\hline CDR documented: yes no & AVDSf: $\mathrm{PaCO}_{2} \div\left(\mathrm{PaCO}_{2}-\mathrm{EtCO}_{2}\right)$ \\
\hline $\begin{array}{l}\text { Type of CDR: Wells; Wells4, Geneva, modified } \\
\text { Geneva; other }\end{array}$ & \\
\hline
\end{tabular}




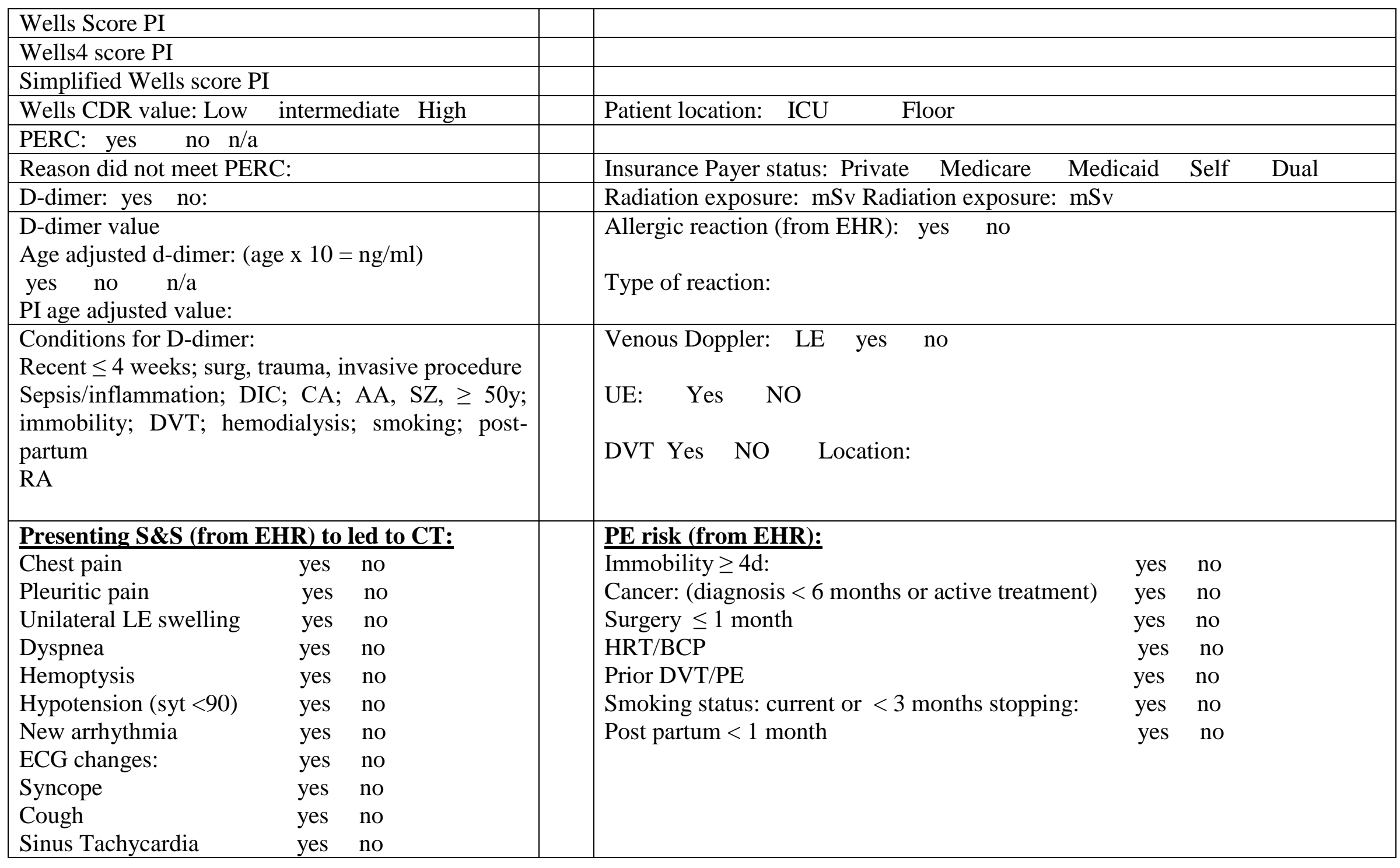




\begin{tabular}{|c|c|c|c|c|}
\hline $\begin{array}{lcc}\text { Increase need for } \mathrm{O} 2 & \text { yes } & \text { no } \\
\text { Low SpO2 } & \text { yes } & \text { no } \\
\text { Other: } & \text { yes } & \text { no }\end{array}$ & & & & \\
\hline $\mathrm{EtCO}_{2}: \quad$ date/time & CIN: (increase in Cr by $25 \%$ at 72 hours) & yes & no & unknown \\
\hline $\begin{array}{lr}\text { PE:(per CT report) } & \text { yes } \\
\text { Indeterminate } & \text { date/time }\end{array}$ & $\mathrm{Cr}$ at baseline (pre-CT) & & & \\
\hline Oxygen: $\quad$ yes $\quad$ no $\quad \mathrm{EtCO}_{2}$ & $\mathrm{Cr}$ at 72 hours post $\mathrm{CT}$ & & & \\
\hline $\begin{array}{l}\text { Type of Oxygen: NC, NRB, HFNC, BiPap, } \\
\text { MV, other }\end{array}$ & Final diagnosis if no PE: & & & \\
\hline
\end{tabular}




\section{APPENDIX E:}

\section{Journal of Vascular Nursing Article Acceptance}

\section{Ref: JVN 201832 R1}

Title: Diagnosis of Pulmonary Embolism: Following the Evidence from Suspicion to Certainty

Journal: Journal of Vascular Nursing

Dear Ms. Prentice,

I am pleased to inform you that your paper has been accepted for publication. My own comments as well as any reviewer comments are appended to the end of this letter. Now that your manuscript has been accepted for publication it will proceed to copy-editing and production.

Thank you for submitting your work to Journal of Vascular Nursing. We hope you consider us again for future submissions.

Kind regards,

Cindy Lewis

Editor

Journal of Vascular Nursing 


\section{APPENDIX F}

\section{Elsevier Author Rights}

\section{Journal author rights}

In order for Elsevier to publish and disseminate research articles, we need publishing rights. This is determined by a publishing agreement between the author and Elsevier. This agreement deals with the transfer or license of the copyright to Elsevier and authors retain significant rights to use and share their own published articles. Elsevier supports the need for authors to share, disseminate and maximize the impact of their research and these rights, in Elsevier proprietary journals* are defined below:

\begin{tabular}{|c|c|}
\hline For subscription articles & For open access articles \\
\hline $\begin{array}{l}\text { Authors transfer copyright to the publisher as part } \\
\text { of a journal publishing agreement, but have the } \\
\text { right to: } \\
\text { - Share their article for Personal Use, Internal } \\
\text { Institutional Use and Scholarly Sharing purposes, } \\
\text { with a DOI link to the version of record on } \\
\text { ScienceDirect (and with the Creative Commons } \\
\text { CC-BY-NC- ND license for author manuscript } \\
\text { versions) } \\
\text { Retain patent, trademark and other intellectual } \\
\text { property rights (including research data). } \\
\text { Proper attribution and credit for the published } \\
\text { work. }\end{array}$ & $\begin{array}{l}\text { Authors sign an exclusive license agreement, where } \\
\text { authors have copyright but license exclusive rights in } \\
\text { their article to the publisher**. In this case authors } \\
\text { have the right to: } \\
\text { - Share their article in the same ways permitted to } \\
\text { third parties under the relevant user license } \\
\text { (together with Personal Use rights) so long as it } \\
\text { contains a CrossMark logo, the end user license, } \\
\text { and a DOI link to the version of record on } \\
\text { ScienceDirect. } \\
\text { Retain patent, trademark and other intellectual } \\
\text { property rights (including research data). } \\
\text { Proper attribution and credit for the published }\end{array}$ \\
\hline
\end{tabular}


VITA

\section{DONNA MARIE PRENTICE}

Donna Marie Prentice was born February 28, 1955 in S. Louis, Missouri and was the second child of James and Edna Prentice. I have live the majority of my life in the greater St. Louis area and attended Eureka High School. I received my diploma in nursing from Lutheran Medical Center School of Nursing, my Bachelor of Science in Nursing and Master of Science in Cardio-Pulmonary Nursing from Saint Louis University in St.

Louis, Missouri. I have spent most of my career working as a staff nurse in intensive care units. When I first became a clinical nurse specialist in 1986, I worked in cardiac rehabilitation and then returned to the intensive care setting in 1989. In 2011, I entered the PhD program at the University Of Missouri Sinclair School Of Nursing as a part time student. As long as I can remember I wanted to be a nurse and I have never once regretted that decision. 